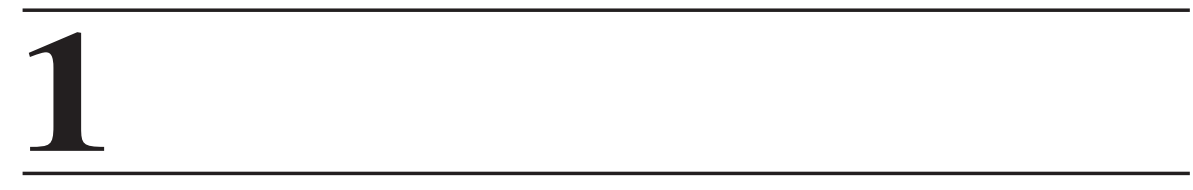

\title{
POLYPHENOLS AND FLAVONOIDS: AN OVERVIEW
}

\author{
Jaime A. Yáñez, Connie M. Remsberg, Jody K. Takemoto, \\ Karina R. Vega-Villa, Preston K. Andrews, Casey L. Sayre, \\ Stephanie E. Martinez, and Neal M. Davies
}

\subsection{INTRODUCTION}

There has been an increase in pharmaceutical and biomedical therapeutic interest in natural products as reflected in sales of nutraceuticals and in the global therapeutic use of traditional medicines. ${ }^{1-9}$ Use of traditional medicines is based on knowledge, skills, and practices based on experiences and theories from different cultures that are used to prevent and maintain health, which may ultimately improve, and/or to treat physical and mental illnesses. ${ }^{10}$ The popularity of these products encompasses almost every aspect of our daily lives from health and beauty, dietary supplements, performance enhancement supplements, food and beverage to overall health and well-being products. ${ }^{1}$ It is apparent that this growing demand for phytotherapies could be very profitable for nutraceutical and pharmaceutical companies. Nutraceutical as well as pharmaceutical companies are interested in many of these naturally occurring compounds that can be extracted from plants and be further modified, synthesized, formulated, manufactured, marketed, and sold for their reported health benefits. Pharmaceutical companies are also using these natural compounds as lead drug candidates that can be modified and formulated to be potential new drug candidates. From drug discovery and development to marketing, between 15 and 20 years may lapse with billions of dollars spent on drug

Flavonoid Pharmacokinetics: Methods of Analysis, Preclinical and Clinical Pharmacokinetics, Safety, and Toxicology, First Edition. Edited by Neal M. Davies and Jaime A. Yáñez.

(C) 2013 John Wiley \& Sons, Inc. Published 2013 by John Wiley \& Sons, Inc. 
development and research of pharmaceuticals. ${ }^{11,12}$ Consumers are looking for beneficial health-related products that have efficacy at a low cost to the consumer, while the nutraceutical industry is struggling to develop therapies at a low cost and to bring them to the market. Through scientific studies, natural products can be scrutinized using pharmaceutical approaches to develop and provide alternative or adjunctive therapies.

The drug discovery process is expensive and time-consuming. It has been estimated to take $10-15$ years and $\$ 800$ million to get a drug to the approval process. ${ }^{13}$ Part of this cost is due to advances in technology whereby drug manufacturers have adopted a target-based discovery paradigm with high throughput screening of compound libraries. This approach, although expected to have vast potential, has not necessarily proven itself. Reviews of new chemical entities have shown that natural products or derivatives of natural products are still the majority of newly developed drugs. For instance, $63 \%$ of the 974 new small molecule chemical entities developed between 1981 and 2006 were directly isolated from nature or semisynthetic derivatives of a natural product. ${ }^{14}$ This trend continues even into this century where approximately $50 \%$ of new small molecule chemical entities approved from 2000 to 2006 have a natural origin..$^{14}$ It is apparent that natural products are important compounds to be explored in the drug discovery process. More importantly, however, there remains a multitude of bioactive compounds yet to be systematically characterized. It is estimated that of the 250,000-750,000 higher plant species, only $10-15 \%$ have been screened for potential therapeutic agents. ${ }^{15}$ Characterizing bioactive molecules in microbial and marine life is even more limited. Nonetheless, natural products remain a reservoir of potential therapeutic agents.

It has been reported that 5000-10,000 compounds are screened before a single drug makes it to the market, and on average, it takes 10-15 years to develop a single drug. ${ }^{16}$ Of the successfully developed drugs, $60 \%$ have a natural origin, either as modified or unmodified drug entities, or as a model for synthetic drugs - not all of them used for human diseases-and it is estimated that $5-15 \%$ of the approximately $250,000-750,000$ species of higher plants have been systematically screened for bioactive compounds. ${ }^{15}$ Structureactivity relationship (SAR) programs are generally employed to improve the chances of phytochemicals being developed as drug entities. ${ }^{17}$ Further studies to develop more drugs of natural origin have been limited in part due to their structural complexity, which is sometimes incompatible with high throughput formats of drug discovery and high extraction costs. ${ }^{16}$ The potentially long resupply time and unforeseen political reasons such as warfare in developing nations also limit the development of plant-based drugs. ${ }^{17}$ As a result, plants remain and represent a virtually untouched reservoir of potential novel compounds. Nevertheless, the number of drugs developed each year based on natural products has remained constant over the last 22 years. ${ }^{17}$

A class of molecules with well-documented therapeutic potential is the polyphenols. Polyphenols are small molecular weight (MW) compounds (MW $200-400 \mathrm{~g} / \mathrm{mol}$ ) that occur naturally. They are produced as secondary 
metabolites that serve to protect the plant from bombardment of pathogens and ultraviolet (UV) radiation. Upon environmental threat, the plant host activates one of the synthesis pathways and polyphenol structures are produced and subsequently secreted. ${ }^{18}$ Which specific polyphenol is produced depends largely on its host, the region of origin, and the environmental stimuli. Many polyphenols are synthesized by the phenylpropanoid pathway. Several classes of polyphenols exist including flavonoids, stilbenes, isoflavonoids, and lignans. Polyphenols of all classes are found in a wide range of plants and plant by-products such as herbal supplements and beauty products.

\subsection{SYNTHESIS}

An understanding of the biosynthesis of natural compounds will enable researchers to further investigate possible therapeutic uses based on the activity of phytochemicals in plants. Plant chemicals are often given the moniker "phytochemicals" and can be classified either as primary or secondary metabolites. ${ }^{19}$ Primary metabolites are widely distributed in nature and are needed for physiological development in plants. On the other hand, secondary metabolites are derived from the primary metabolites, are limited in distribution in the plant kingdom, and are restricted to a particular taxonomic group (Fig. 1.1). Secondary metabolites usually play an ecological role; for example, they act as pollinator attractants, are involved in chemical defense, are often end

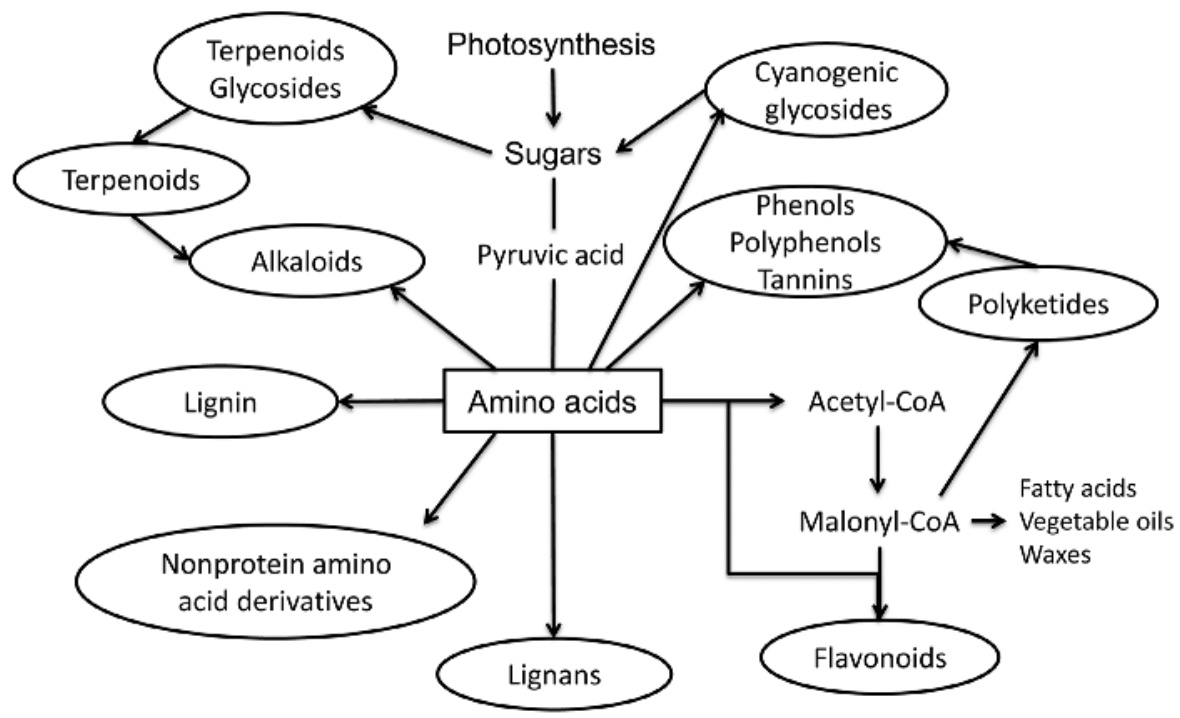

Figure 1.1. Biosynthetic origin of some plant-derived compounds. Major groups of secondary metabolites are indicated by ovals. 


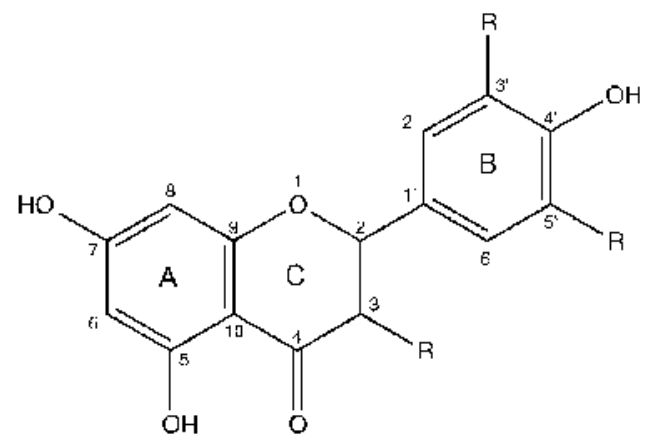

Figure 1.2. Basic chemical structure and numbering pattern of flavonoids.

products from chemical adaptations to environmental stresses, or are synthesized in specialized cell types at different developmental stages of plant development or during disease or are induced by sunlight. ${ }^{19}$

Allelochemicals are phytotoxic compounds produced by higher plants that include flavonoids. Like other secondary metabolites, flavonoids have complex structures where multiple chiral centers are common. ${ }^{19}$ Flavonoids consist of a $\mathrm{C}_{15}$ unit with two benzene rings $\mathrm{A}$ and $\mathrm{B}$ connected by a three-carbon chain (Fig. 1.2). This chain is closed in most flavonoids, forming the heterocyclic ring $\mathrm{C}$; however, chalcones and dihydrochalcones present as an open ring system. ${ }^{20}$ Depending on the oxidation state of the $\mathrm{C}$ ring and on the connection of the $\mathrm{B}$ ring to the $\mathrm{C}$ ring, ${ }^{21}$ flavonoids can be classified into various subclasses. Flavonoids can undergo hydroxylation, methylation, glycosylation, acylation, prenylation, and sulfonation; these basic chemical metabolic substitutions generate the different subclasses: flavanols, flavanones, flavones, isoflavones, flavonols, dihydroflavonols, and anthocyanidins. ${ }^{20,21}$ Flavonoids in nature are naturally most often found as glycosides and other conjugates; likewise, many flavonoids are polymerized by plants themselves or as a result of food processing. ${ }^{21}$

\subsubsection{Synthesis of Flavonoids}

In plants, primary metabolites such as sugar are associated with basic life functions including, but not limited to, cell division, growth, and reproduction. ${ }^{22} \mathrm{On}$ the other hand secondary metabolites are involved in the adaptive necessity of plants to their environments, such as pigmentation, defense from toxins, and enzyme inhibition, ${ }^{23-25}$ additionally, these secondary metabolites can have pathogenic or symbiotic effects. ${ }^{26}$ Secondary metabolites including polyphenols have been associated with having many health benefits. ${ }^{27}$ The abundance of polyphenols in foodstuffs is apparent, although they often have not been adequately characterized; however, an assortment of polyphenols is prevalent in unprocessed and processed foods and beverages and nutraceuticals. ${ }^{28}$ 


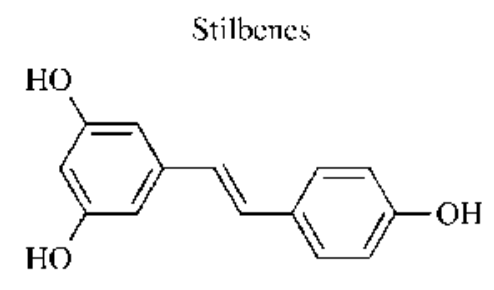

Lignatis<smiles>COCc1cc(CC(CO)C(CO)Cc2ccc(O)c(OC)c2)ccc1O</smiles>

Hydroxybenzide Acids<smiles>[R]c1cc(C(=O)O)cc([R])c1[R]</smiles>

Hydroxycinnatuic Acids<smiles>[R]c1ccc(/C=C/C(=O)O)cc1[R]</smiles>

Figure 1.3. Chemical structures of polyphenols. Representative chemical structures of hydroxybenzoic acids, hydroxycinnamic acids, flavonoids, ligands, and stilbenes. Adapted from Manach et al. ${ }^{63}$

Structurally, polyphenols or phenolics have one or more aromatic rings with hydroxyl groups and can occur as simple and complex molecules. ${ }^{29}$ Polyphenols can be subdivided into two major groups: hydroxybenzoic acids and hydroxycinnamic acids (Fig. 1.3). Examples of hydroxybenzoic acids include gallic and vanillic acids. They are typically found in the bound form as a smaller entity of a ligand or tannin or are linked to a sugar or an organic acid in plant foods. ${ }^{25}$ Alternatively, hydroxycinnamic acid examples include p-coumaric and caffeic acids. These molecules are found esterified with small molecules, bound to cell walls, and/or proteins. ${ }^{25} \mathrm{~A}$ subcategory of p-coumaric acid derivatives is the flavonoids (flavonones, flavanones, flavonols, flavanols [proanthocyanidins, catechins, epicatechins, procyanidins, prodelphinidins], and anthocyanins) as these are the most abundant polyphenols in our diets (Fig. 1.4). ${ }^{30,31}$ Flavonones and isoflavones can be predominantly found in citrus fruits and soy products, respectively. Proanthocyanidins are complex polymeric flavanols found in conjunction with flavanol catechins from apples, pears, grape, and chocolate products; these flavonoids are primarily responsible for the astringency of foods. Anthocyanins are located in an assortment of fruits (cherries, 
ILANAN 3 OLS $(n=1)$

PROANTIOCYANIDINS $(n>1)$<smiles>[R]c1cc(C2Oc3cc(O)cc(O)c3C[C@H]2O)cc([R])c1O</smiles><smiles>[R15]O[Y16]#[SH]</smiles>

ISOFLAVONES<smiles>[R]c1cc(-c2coc3cc(O)cc(O)c3c2=O)cc([R])c1O</smiles><smiles>[R]c1cc(/C=C/C(=O)c2c(O)cc(O)cc2O)cc([R])c1O</smiles>

FLAVONES $(n=1)$

CONDENSED TANNLNS $(n>1)$<smiles>[R]c1cc(-c2cc(=O)c3c(O)cc(O)cc3o2)cc([R])c1O</smiles><smiles></smiles><smiles>[R]c1cc(C2CC(=O)c3c(O)cc(O)cc3O2)cc([R])c1O</smiles><smiles>[R]c1cc(-c2oc3cc(O)cc(O)c3c(=O)c2O)cc([R])c1O</smiles>

Figure 1.4. General structures for polyphenols.

plums, strawberries, raspberries, blackberries, and currants). In addition to these polyphenol subclasses, in nature, flavonoids are also prevalent as a glycoside (parent compound or aglycone with a sugar moiety attached) as this sugar moiety helps to facilitate water solubility and transportability of the aglycone. ${ }^{26,32,33}$ Another important factor to consider is that the distribution of polyphenols in plant tissues is heterogenous; thus, the seed, pericarp, flavedo, and albedo contain polyphenols in different proportions. ${ }^{31}$

Flavonoids are synthesized via the phenylpropanoid pathway and are derived from estrogen. ${ }^{34}$ The phenylalanine structure from phenolic compounds is transformed to cinnamate by the enzyme phenylalanine ammonialyase (PAL). The cinnamate 4-hydroxylase $(\mathrm{C} 4 \mathrm{H})$ converts cinnamate to 


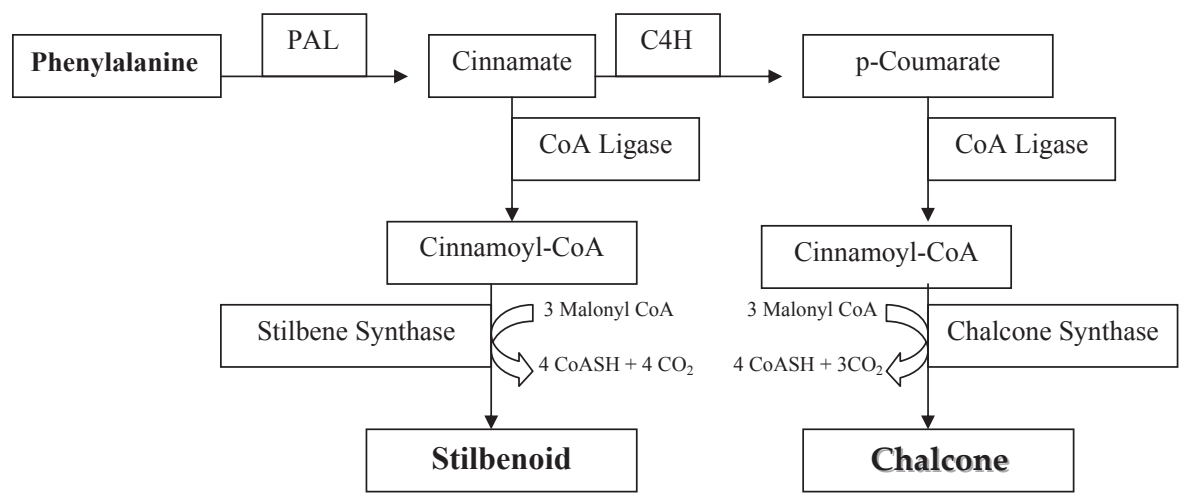

Figure 1.5. Phenylpropanoid pathway and chalcone synthesis.

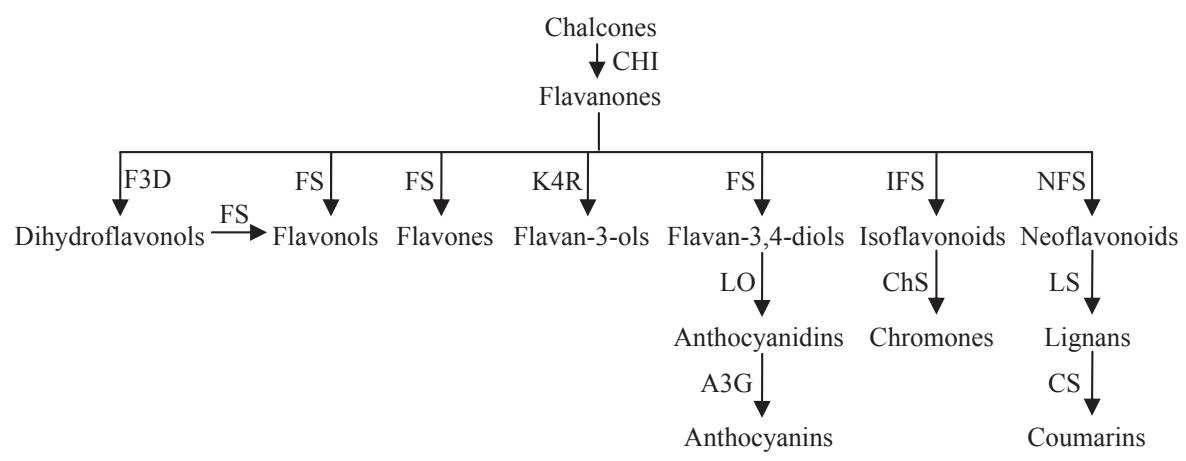

Figure 1.6. Synthesis pathway of chiral flavanones and other flavonoid derivatives. $\mathrm{CHI}$, chalcone isomerase; $\mathrm{K} 4 \mathrm{R}$, kaempferol-4-reductase; FLS, flavonol synthase; F3D, flavanone-3-dioxygenase; IFS, isoflavonoid synthase; NFS, neoflavonoid synthase; LO, leucocyanidin oxygenase; $\mathrm{A} 3 \mathrm{G}$, anthocyanidin-3-O-glucosyltransferase; $\mathrm{ChS}$, chromone synthase; LS, lignan synthase; CS, coumarin synthase.

p-coumarate, and then an acetyl-CoA group is added by the CoA ligase enzyme to yield cinnamoyl-CoA. Lastly, this product is transformed by chalcone synthase (CHS) to yield a general chalcone structure. Stilbenoids are synthesized in much the same fashion except for the $\mathrm{C} 4 \mathrm{H}$ enzymatic step (Fig. 1.5).

The chalcone structure is further metabolized by the chalcone isomerase (CHI) to the general chiral flavanone structure. From the general chiral flavanone structure, the other derivatives, namely, dihydroflavonols, flavonols, flavones, flavan-3-ols, flavan-3,4-diols, isoflavonoids, and neoflavonoids, are further metabolized by a well-characterized enzymatically derived process (Fig. 1.6). Anthocyanidins and anthocyanins are derived from flavan-3,4-diols by leuocyanidin oxygenase (LO) and anthocyanidins-3-O-glucosyltransferase, respectively. Chromones are synthesized from isoflavonoids through the chromone synthase $(\mathrm{ChS})$, while lignans and coumarins are derived from 


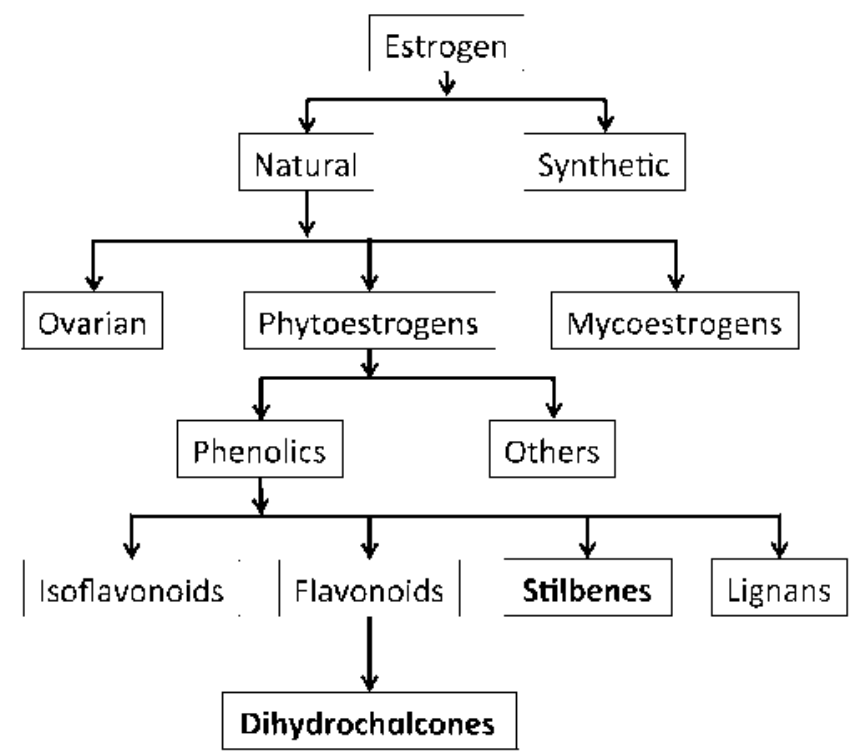

Figure 1.7. Relationship between stilbenes and dihydrochalcones to other polyphenols.

neoflavonoids by lignan synthase (LS) and coumarin synthase (CS), respectively (Fig. 1.6).

In addition to flavanone, other small natural compounds found in a wide variety of food and plant sources exist. These compounds, namely, flavonoids, isoflavonoids, and lignans, have generated much scientific interest in their potential clinical applications in the possible dietary prevention of different diseases. Flavanones, stilbenes, lignans, isoflavonoids, and other flavonoid derivatives are similar in structure and provide host-protective purposes. They share the common parent compound, estrogen, in their synthesis and are differentiated based on key structural differences, specific plant hosts, and the environment (Fig. 1.7).

\subsection{SOURCES}

In 1936, Professor Szent-Györgyi reported the isolation of a substance that was a strong reducing agent acting as a cofactor in the reaction between peroxidase and ascorbic acid. This substance was initially given the name "vitamin P"; this substance has been subsequently categorized as the flavonoid rutin. Professor Szent-Györgyi's seminal investigations identified rutin and reported its isolation from both lemons and red pepper. ${ }^{35}$ Since this time, more than other 4000 flavonoids have being identified and studied. Flavonoids are a group of polyphenolic compounds of low $\mathrm{MW}^{36}$ that present a common benzo- $\gamma$-pyrone structure. ${ }^{37}$ They are categorized into various subclasses 
including flavones, flavonols, flavanones, isoflavanones, anthocyanidins, and catechins.

Consumption of polyphenols could be close to $1 \mathrm{~g}$ /day in our diet, making polyphenols the largest source of antioxidants. ${ }^{38}$ Dietary sources of polyphenols include fruits, vegetables, cereals, legumes, chocolate, and plant-based beverages such as juices, tea, and wine. ${ }^{38}$ Extensive biomedical evidence suggests that polyphenolic compounds no matter their class may contribute to the prevention of cardiovascular disease, cancer, osteoporosis, diabetes, and neurodegenerative diseases. ${ }^{39-41}$ As polyphenols are found in plant sources consumed regularly or that are used in traditional medicine, there is a necessity to study these potentially beneficial compounds. Additionally, potential health benefiting properties such as antiinflammatory, antiproliferative, and colon protection may call for development of these compounds into future therapeutic agents. The average human diet contains a considerable amount of flavonoids, the major dietary sources of which include fruits (i.e., orange, grapefruit, apple, and strawberry), vegetables (i.e., onion, broccoli, green pepper, and tomato), soybeans, and a variety of herbs. ${ }^{42,43}$ Due to the constant and significant intake of these compounds in our diet, the United States Department of Agriculture (USDA) has created a database that contains the reported average content of these compounds in different foodstuffs. ${ }^{44}$ Among the classes of flavonoids, flavanones have been defined as citrus flavonoids ${ }^{44-46}$ due to their almost unique presence in citrus fruits. ${ }^{44,47-57}$ However, flavanones have been also reported in tomatoes, ${ }^{35,58-60}$ peanuts, ${ }^{61,62}$ and some herbs such as mint, ${ }^{63}$ gaviota tarplant, ${ }^{62,64}$ yerba santa, ${ }^{62,65}$ and thyme. ${ }^{62,66}$ Flavonoids are consumed in the human diet; the calculated flavonoid intake varies among countries since cultural dietary habits, available flora, and weather influence what food is consumed and, therefore, the amount and subclasses of flavonoids ingested. ${ }^{21}$ However, in the Western diet, the overall amount of flavonoids consumed on a daily basis is likely in the milligram range. It has been determined that the consumption of selected subclasses of flavonoids may be more important in determining health benefits than the total flavonoid intake. The content of flavonoids is also potentially influenced by food processing and storage conditions, which can result in transformation of flavonoids, and loss of flavonoid content. ${ }^{21}$

Flavonoids in general have been studied for more than 70 years in in vivo and in vitro systems. They have been shown to exert potent antioxidant activity ${ }^{48,59,67-69}$ in some instances, stronger than $\alpha$-tocopherol (vitamin E). ${ }^{70}$ They have been also shown to exhibit beneficial effects on capillary permeability and fragility, ${ }^{23,37,48,68,71-77}$ to have antiplatelet, ${ }^{23,37,48,67,68,71-76}$ hypolipidemic, ${ }^{67,78-81}$ antihypertensive, ${ }^{51,67,82}$ antimicrobial, ${ }^{67}$ antiviral, ${ }^{23,37,48,67,68,71-76,83,84}$ antiallergenic, ${ }^{85}$ antiulcerogenic, ${ }^{67}$ cytotoxic, ${ }^{67}$ antineoplastic, ${ }^{47,50,67,86-90}$ antiinflammatory, ${ }^{23,37,48,67,68,71-76}$ antiatherogenic, ${ }^{67,91}$ and antihepatotoxic ${ }^{67}$ activities. There are multiple chiral flavanones; however, they have been generally thought of as achiral entities and their chiral nature, in many cases, has not been recognized or denoted. Furthermore, the USDA database reports these 
compounds as achiral entities and uses the aglycone terminology interchangeably with the glycosides. ${ }^{92}$

The importance of considering the chiral nature of naturally occurring compounds and xenobiotics has been previously reviewed by Yáñez et al. ${ }^{93}$ The chirality of flavonoids was initially examined by Krause and Galensa's studies in the early 1980s. ${ }^{62,94,95}$ Chirality plays an important role in biological activity; disciplines like agriculture, nutrition, and pharmaceutical sciences have long recognized the existence of natural chiral compounds; however, developed methods of analysis have often failed to stereospecifically separate and discriminate compounds into their respective antipodes. The advantage of chiral separation methods includes a more thorough appreciation of the stereospecific disposition of natural compounds including flavonoids. Moreover, the lack of configurational stability is a common issue with chiral xenobiotics. Some chiral flavonoids have been reported to undergo nonenzymatic interconversion of one stereoisomer into another in isomerization processes such as racemization and enantiomerization..$^{93}$ Racemization refers to the conversion of an enantioenriched substance into a mixture of enantiomers. Alternatively, enantiomerization refers to a reversible interconversion of enantiomers. The importance of isomerization in stereospecific chromatography as well as in the pharmaceutical manufacturing process has been described. ${ }^{93}$ Therefore, the development of chiral methodology to analyze this kind of xenobiotics is necessary.

The study of the stereochemistry of flavonoids comprises mainly C-2 and C-3; nevertheless, the majority of natural flavonoids possess only one stereochemical isomer at the C-2 position. C-2 and C-3 act as chiral centers of dihydroxyflavonols and are important in flavonoid metabolism. The nomenclature of flavonoids with two chiral centers remains a topic of debate since the use of symbolism (+/-) or 2,3-cis or -trans seems to be inadequate to describe four possible enantiomers. ${ }^{96}$ It is also argued that the R, S nomenclature for absolute configuration is confusing for flavonoids because the designation of $\mathrm{R}$ or $\mathrm{S}$ changes at $\mathrm{C}-2$ depending on the priority of neighboring groups, even though the stereochemistry remains the same. ${ }^{96}$ An alternative nomenclature system was proposed by Hemingway et al. ${ }^{97}$ based on that used for carbohydrate chemistry. In this system, the prefix ent- has been used for the mirror images. However, scientific consensus has not been reached on stereochemical lexicon cognates, and, to date, all these systems of nomenclature still remain being used and appearing in the biomedical, biochemical, agricultural, and food science literature.

\subsection{PHARMACOLOGICAL ACTIVITIES OF SELECTED FLAVONOIDS}

Humans have utilized and/or consumed polyphenols for health benefits. For centuries, alternative medicine has been practiced in different countries as 
exemplified by the use of plant extracts as traditional medicinal folk agents in the prevention and treatment of an assortment of ailments like menses, coughing, digestive problems, and so on. There are a variety of health benefits that can be attributed to the use/consumption of polyphenols including antioxidant, anticancer, antihyperlipidemic, antiallergenic, antibacterial, antiviral, and antiinflammatory. ${ }^{25}$ Conversely, there are also toxic effects associated with the use/consumption of polyphenols such as anemia due to the inhibited absorption of nutrients and minerals and inhibitory effects on cytochrome P450 enzymes (P450) resulting in potential drug-drug interactions. Current uses of polyphenols, in addition to their dietary health-related benefits and herbal remedies, are their use as dietary supplements and as pharmaceutical leads; thus, the reported intake of polyphenols is in the tens to hundreds of milligrams per day in human diets. ${ }^{21,31}$

The World Health Organization (WHO), published a comprehensive study and analysis in September 2008 naming the leading causes of mortality in the world in 2004 to include cardiovascular and pulmonary ailments and cancer accounting for approximately 22.9 million deaths. ${ }^{98}$ These statistics remain consistent with the data published in 2007 with similar primary causes of mortality as seen in $2002 .{ }^{99}$ There appears to be evidence that suggests that the leading causes of death are often multifactorial and intertwined, for example, dyspnea, malignant pericardial effusion, malignant pleural effusion, and superior vena cava syndrome, all of which are cardiopulmonary and/or vascular problems. ${ }^{100}$ Biomedical literature suggests etiologies of cardiovascular and pulmonary ailments and cancer have been linked to diet and nutrition, environment, exercise, genetics, hormones, lifestyles, radiation, sex, and weight; however, direct correlations of the disease, etiologies, and pathogenic mechanisms have not been fully elucidated. Contemporary Western medicine provides a variety of options to prevent and treat cardiovascular and pulmonary ailments and cancer. It is becoming increasingly popular and apparent that there is a need for other effective means to prevent, treat, and develop newer drugs or alternatives to disease treatment for both the consumer and the nutraceutical and pharmaceutical industry at a lower cost.

There are a several assay methodologies to determine the total polyphenolic content of a sample through the use of the Folin-Denis and Folin-Ciocalteu reagents and complexation with aluminum III ion. ${ }^{101-103}$ The Folin-Denis or Folin-Ciocalteu reducing reagents are able to form phosphomolybdicphosphotungstic-phenol complexes, which can be monitored at a visible wavelength of $760 \mathrm{~nm}$ via reduction-oxidation reaction. These assays may have some inherent falsely elevated values because of interference as there may be other components in the sample that are also reducing reagents. As previously mentioned, the total phenolic content of the sample can be quantified; thus, this method is a nonspecific measurement of polyphenol content. Alternatively, complexation of polyphenols with aluminum III ion can be used to determine the quantity of polyphenols in the sample monitored at a wavelength of $425 \mathrm{~nm}$. This method is dependent upon the aluminum ion 
<smiles>COC1=CC[C@@H]([C@@H]2CC(=O)C3C(O)=CC(O[C@@H]4O[C@H](CO[C@@H]5O[C@@H](C)C[C@H](O)[C@H]5O)[C@@H](O)[C@H](O)[C@H]4O)=CC3O2)C=C1O</smiles>

Figure 1.8. Structure of hesperidin. The asterisk (*) denotes a chiral center.<smiles>COc1ccc(C2CC(=O)c3c(O)cc(O)cc3O2)cc1O</smiles>

Figure 1.9. Structure of hesperetin. The asterisk (*) denotes a chiral center.

complexing with the carbonyl and hydroxyl groups of the polyphenol. Again, these processes are not specific for a particular polyphenol; therefore, it is necessary to develop analytical methods to quantify individual polyphenols in a sample to enable determination of a correlation between the amount of a polyphenol in a sample and a health-related benefit.

\subsubsection{Hesperidin and Hesperetin}

Hesperidin ((+/-) 3,5,7-trihydroxy-4'-methoxyflavanone 7-rhamnoglucoside) $\mathrm{C}_{28} \mathrm{H}_{34} \mathrm{O}_{15}$, MW $610.56 \mathrm{~g} / \mathrm{mol}$, experimental octanol to water partition coefficient $(\mathrm{X} \log \mathrm{P})$ value of -1.1 (Fig. 1.8), is a chiral flavanone-7-O-glycoside consumed in oranges and in other citrus fruits and herbal products. ${ }^{104}$ The rutinose sugar moiety is rapidly cleaved off the parent compound to leave the aglycone bioflavonoid hesperetin (+/-3,5,7-trihydroxy-4'-methoxyflavanone) $\mathrm{C}_{16} \mathrm{H}_{14} \mathrm{O}_{6}, \mathrm{MW} 302.28 \mathrm{~g} / \mathrm{mol}$, XLogP value of 2.174 (Fig. 1.9), also a chiral flavonoid. There is current interest in the medical use of bioflavonoids, including hesperetin, in the treatment of a variety of cancers and vascular diseases. $^{105}$

1.4.1.1 Antifungal, Antibacterial, and Antiviral Activity Hesperidin extracted from grapefruit (Citrus paradise Macf., Rutaceae) seed and pulp ethanolic extracts has been related to have antibacterial and antifungal activity against 20 bacterial and 10 yeast strains. ${ }^{106}$ The level of antimicrobial effects was assessed employing an in vitro agar assay and standard broth dilution susceptibility test. It was observed that hesperidin exhibits strong antimicrobial activity against Salmonella enteritidis (minimum inhibitory concentration [MIC] of $2.06 \%$ extract concentration-m/V), while its activity against other bacteria and yeasts ranged from $4.13 \%$ to $16.5 \% \mathrm{~m} / \mathrm{V} \cdot{ }^{106}$ Furthermore, hesperi- 
din has also been observed to have protective effects in infected mice with encephalomyocarditis (EMC) virus and Staphylococcus aureus that were administered with hesperidin before or coadministered with the lethal viralbacterial dose. ${ }^{107}$

In the case of the aglycone hesperetin, it has been shown to have MIC $>20 \mu \mathrm{g} / \mathrm{mL}$ against Helicobacter pylori. However, neither hesperetin nor other flavonoids and phenolic acids inhibited the urease activity of H. pylori. ${ }^{108}$ Furthermore, hesperetin has shown to be an effective in vitro agent against severe acute respiratory syndrome (SARS) (or similar) coronavirus (CoV) infections. ${ }^{109}$ Hesperetin inhibits the SARS-CoV replication by interacting with the spike (S) glycoprotein (S1 domain) in the host cell receptor and fusing the S2 domain with the host cell membrane activating the replicase polyproteins by the virus-encoded proteases (3C-like cysteine protease [3CLpro] and papain-like cysteine protease) and other virus-encoded enzymes such as the NTPase/helicase and RNA-dependent RNA polymerase. The blocking of the S1 may play an important role in the immunoprophylaxis of SARS. ${ }^{109}$ Similar activities have also been observed for hesperetin against the replication of the neurovirulent Sindbis strain (NSV) having $50 \%$ inhibitory doses $\left(\mathrm{ID}_{50}\right)$ of $20.5 \mu \mathrm{g} / \mathrm{mL}$. However, its glycoside, hesperidin, did not have inhibitory activity, indicating the possibility that the rutinose moiety of flavanones blocks the antiviral effect. ${ }^{110}$ Nevertheless, hesperetin has also been reported to be effective against the replication of herpes simplex virus type 1 (HSV-1), poliovirus type 1 , parainfluenza virus type 3 (Pf-3), and respiratory syncytial virus (RSV) in in vitro cell culture monolayers employing the technique of viral plaque reduction. ${ }^{83}$

1.4.1.2 Antiinflammatory Activity The inflammatory process involves a series of events encompassed by numerous stimuli such as infectious agents, ischemia, antigen-antibody interactions, and chemical, thermal, or mechanical injury. The inflammatory responses have been characterized to occur in three distinct phases, each apparently mediated by different mechanisms: an acute phase characterized by local vasodilatation and increased capillary permeability, a subacute phase characterized by infiltration of leukocyte and phagocyte cells, and a chronic proliferative phase, in which tissue degeneration and fibrosis occur. ${ }^{111}$ Different animal models have been developed to study the different phases of an inflammatory response. In the case of testing acute inflammatory response, the carrageenan-induced paw edema in mice ${ }^{112}$ and the xylene-induced ear edema ${ }^{113}$ are widely employed. Methods to test the proliferative phase (granuloma formation) include the cotton pellet granuloma model ${ }^{114}$ Another model that allows the assessment of acute and chronic inflammation is the adjuvant-carrageenan-induced inflammation (ACII) model to induce adjuvant arthritis. ${ }^{115}$ Hesperidin and hesperetin were tested under these models, and it was observed that only hesperetin had a positive effect in reducing the carrageenan-induced paw edema in mice by $48 \%$ and $29 \%$ after 3 and 7 hours postinflammatory insult. ${ }^{111}$ In the case of the 
xylene-induced ear edema model, both hesperidin and hesperetin had a positive effect by reducing the edema by $45 \%$ and $44 \%$, respectively. ${ }^{111}$ Similar observations were observed in the cotton pellet granuloma, whereas hesperidin and hesperetin inhibited granuloma formation by $30 \%$ and $28 \%$, respectively. ${ }^{111}$ In the case of the ACII model, hesperidin exhibited activity in the acute phase (day 6) by causing a reduction in paw edema of $52 \%$ and exhibited a more moderate reduction in the chronic phase (7-21 days) by reducing the paw edema by $36 \%, 44 \%, 47 \%, 38 \%$, and $31 \%$ at $7,8,10,12$, and 16 days postinflammatory insult, respectively. ${ }^{111}$ Different mechanisms to elucidate how hesperidin, hesperetin, and other polyphenols might carry their antiinflammatory activity have been proposed. Among these, it has been observed that after carrageenan injection, there is an initial release of histamine and serotonin during the first 1.5 hours with a posterior release of kinin between 1.5 and 2.5 hours, followed with a release of prostaglandins until 5 hours. ${ }^{116-118}$ Thus, it is believed that hesperidin and hesperetin might be involved with a variety of steps during the development of inflammation.

Other studies have reported that hesperidin downregulates the lipopolysaccharide (LPS)-induced expression of different proinflammatory (tumor necrosis factor-alpha [TNF- $\alpha$ ], IL-1 beta, interleukin-6 [IL-6]) and antiinflammatory mediators (IL-12), cytokines as well as cytokines (KC, MCP-1 and MIP-2), while enhancing the production of other antiinflammatory cytokines (IL-4 and IL-10). ${ }^{119}$ In this study, mice were challenged with intratracheal LPS $(100 \mu \mathrm{g})$ 30 minutes before treatment with hesperidin $(200 \mathrm{mg} / \mathrm{kg}$ oral administration) or vehicle. After 4 and 24 hours, bronchoalveolar lavage fluid was collected, observing that hesperidin significantly reduced the total leukocyte counts, nitric oxide production, and inducible nitric oxide synthase (iNOS) expression. ${ }^{119}$ These results correlate with in vitro studies that have demonstrated that hesperidin suppresses the expression of IL-8 on A549 cells and THP-1 cells, the expression of TNF- $\alpha$, IL- 1 beta, and IL- 6 on THP- 1 cells, and the expression of intercellular adhesion molecule-1 (ICAM-1) and vascular cell adhesion molecule-1 (VCAM-1) (responsible for cell adhesion) on A549 cells. The suppression of these inflammatory mediators is regulated by nuclear factor-kappa B (NF- $\kappa \mathrm{B})$ and $\mathrm{AP}-1$, which are activated by I $\kappa \mathrm{B}$ and mitogenactivated protein kinase (MAPK) pathways, indicating that hesperidin might interact within these pathways to exert its antiinflammatory activity. ${ }^{119}$

1.4.1.3 Antioxidant Activity Hesperidin and its aglycone, hesperetin, have been assessed in various in vitro chemical antioxidant models (cell-free bioassay systems). It has been observed that both hesperidin and hesperetin exhibited similar patterns of 1,1-diphenyl-2-picrylhydrazyl (DPPH) radical scavenging activities. ${ }^{120}$ Similar results have been reported elsewhere for hesperidin, an antioxidant that was comparable in efficacy to Trolox ${ }^{\circledR}$ (positive control). ${ }^{121}$ Furthermore, hesperetin alone has been reported to effectively scavenge peroxynitrite $\left(\mathrm{ONOO}^{-}\right)$in a concentration-dependent manner. Peroxynitrite $\left(\mathrm{ONOO}^{-}\right)$is a reactive oxidant formed from superoxide $\left({ }^{*} \mathrm{O}_{2}^{-}\right)$and 
nitric oxide $(* \mathrm{NO})$, which can oxidize several cellular components, including essential protein, nonprotein thiols, DNA, low density lipoproteins (LDLs), and membrane phospholipids. ${ }^{122}$

Both hesperidin and hesperetin have also been assessed for their antioxidant capacity in vivo. It has been observed that hesperidin $(25 \mathrm{mg} / \mathrm{kg}$ body weight [BW] p.o.) offers protection against lung damage induced by a subcutaneous injection of nicotine at a dosage of $2.5 \mathrm{mg} / \mathrm{kg} \mathrm{BW}$ for 5 days a week. Hesperidin treatment resulted in a decreased level of all the marker enzymes, the recovery of the in vivo antioxidant status back to near baseline level, ${ }^{123}$ and different matrix metalloproteinases (MMPs) were downregulated. ${ }^{124} \mathrm{Hes}-$ peridin $(60 \mathrm{mg} / \mathrm{kg} \mathrm{BW} /$ day p.o. for 9 days) has also been shown to increase the free SH-group concentration (SHC), hydrogen-donating ability (HDA), and natural scavenger capacity, and to decrease the hepatic malonaldehyde content and dien conjugate (DC) in male Wistar albino rats with alimentary-induced fatty livers. ${ }^{125}$ Furthermore, hesperidin in the same animal models has been reported to increase both the total scavenger capacity (TSC) and the activity of superoxide dismutase (SOD) in liver homogenates, and to induce slight changes in the $\mathrm{Cu}, \mathrm{Zn}, \mathrm{Mn}$, and $\mathrm{Fe}$ contents of liver homogenates. ${ }^{126}$ Similar results were observed for hesperidin (100 and $200 \mathrm{mg} / \mathrm{kg}$ p.o. for 1 week) in $\mathrm{CCl}_{4}$-induced oxidative stressed rats, whereas the thiobarbituric acid-reactive substances (TBARSs) decreased and the glutathione (GSH) content, SOD, and catalase (CAT) levels increased in liver and kidney homogenates. ${ }^{127}$ In the case of hesperetin, it was observed to be a potent antioxidant, inhibiting lipid peroxidation initiated in rat brain homogenates by $\mathrm{Fe}^{2+}$ and $\mathrm{L}$-ascorbic acid. Hesperetin was found to protect primary cultured cortical cells against the oxidative neuronal damage induced by $\mathrm{H}_{2} \mathrm{O}_{2}$ or xanthine and xanthine oxidase (XO). In addition, it was shown to attenuate the excitotoxic neuronal damage induced by excess glutamate in the cortical cultures. ${ }^{120}$

1.4.1.4 Anticancer Activity In vitro tests have shown that hesperidin reduces the proliferation of many cancer cells. ${ }^{128}$ For instance, hesperidin $(100 \mu \mathrm{M})$ has been shown to reduce the cell viability $(65 \pm 0.05 \%)$ of human colon cancer cells, SNU-C4 based in 3-(4,5-dimethylthiazol-2-yl)-2,5diphenyltetrazolium bromide (MTT) assay. ${ }^{129}$ It was proposed that hesperidin treatment decreased the expression of B-cell CLL/lymphoma 2 (BCL2) mRNA and increased the expression of BCL2-associated X protein (BAX) and of the apoptotic factor caspase-3 (CASP3) inducing apoptosis. ${ }^{129}$ Another study, less mechanistic in nature, observed that hesperidin and hesperetin at smaller concentrations $(1 \mu \mathrm{M})$ inhibit the neoplastic transformation of $\mathrm{C} 3 \mathrm{H}$ $10 \mathrm{~T} 1 / 2$ murine fibroblasts induced by the carcinogen 3-methylcholanthrene. ${ }^{130}$

Hesperetin has been reported to affect the proliferation and growth of a human breast carcinoma cell line, MDA-MB-435, with an $\mathrm{IC}_{50}$ of $22.5 \mu \mathrm{g} / \mathrm{mL}$ and to exhibit low cytotoxicity ( $>500 \mu \mathrm{g} / \mathrm{mL}$ for $50 \%$ cell death) ${ }^{88}$ Furthermore, hesperetin has also been reported to significantly inhibit cell proliferation of MCF-7 cells in a concentration-dependent manner by causing cell cycle 
arrest in the G1 phase. In the G1 phase, hesperetin downregulates the cyclindependent kinases (CDKs) and cyclins while upregulating p21(Cip1) and p27(Kip1) in MCF-7 cells. Hesperetin also decreases CDK2 and CDK4 together with cyclin D. In addition, hesperetin increases the binding of CDK4 with $\mathrm{p} 21$ (Cip1) but not $\mathrm{p} 27$ (Kip1) or p57(Kip2), indicating that the regulation of CDK4 and p21(Cip1)1 may participate in the anticancer activity pathway of hesperetin in MCF-7 cells. ${ }^{131}$

The $\mathrm{Apc}^{\mathrm{Min} /+}$ mouse model and the azoxymethane (AOM) rat model are the main animal models used to study the effect of dietary agents on colorectal cancer. ${ }^{132}$ Different chemopreventive agents in the AOM rat model have been analyzed, ${ }^{132,133}$ and it was observed that hesperidin and hesperetin-rich foods are able to suppress colon adenocarcinoma and/or consistently inhibit adenoma and aberrant crypt foci (ACF) in several independent rat studies. ${ }^{90,132,134-136}$ Other animal studies have reported that hesperidin has the capacity to inhibit tumor initiation and promotion in CD-1 mice skin. Subcutaneous application of hesperidin did not inhibit 7,12-dimethylbenz(a)anthracene-induced tumor initiation but did inhibit 12-O-tetradecanoyl-13-phorbol acetate-induced tumor promotion. ${ }^{137}$ Furthermore, male imprinting control (ICR) mice that were N-butyl-N-(4-hydroxybutyl)nitrosamine (OH-BBN) $\quad(500 \mu \mathrm{g} / \mathrm{mL})$ induced for urinary bladder tumors were fed with hesperidin $(1 \mathrm{mg} / \mathrm{mL})$, diosmin $(1 \mathrm{mg} / \mathrm{mL})$, and combination $(4.9 \mathrm{mg} / \mathrm{mL}$ diosmin and $0.1 \mathrm{mg} / \mathrm{mL}$ hesperidin) for 8 weeks. It was observed that hesperidin and diosmin alone or in combination significantly reduced the frequency of bladder carcinoma and preneoplasia. Also, a significant decrease in the incidence of bladder lesions and cell-proliferation activity estimated by enumeration of silver-stained nucleolar-organizer-region-associated proteins (AgNORs) and by the 5bromodeoxyuridine (BUdR)-labeling index was observed. ${ }^{90}$ However, other research groups have observed that hesperidin $(100 \mu \mathrm{g} / \mathrm{mL})$ and diosmin $(100 \mu \mathrm{g} / \mathrm{mL})$ alone or in combination $(900 \mu \mathrm{g} / \mathrm{mL}$ diosmin and $100 \mu \mathrm{g} / \mathrm{mL}$ hesperidin) provide no pathological alterations during the initiation and postinitiation phases of esophageal carcinogenesis initiation with $\mathrm{N}$-methyl-Namylnitrosamine (MNAN) in male Wistar rats. ${ }^{138}$

1.4.1.5 Cyclooxygenase-1 and-2 Inhibitory Activity Hesperidin has been assessed for its inhibitory effect on LPS-induced overexpression of cyclooxygenase-2 (COX-2), iNOS proteins, overproduction of prostaglandin $\mathrm{E}_{2}\left(\mathrm{PGE}_{2}\right)$ and nitric oxide (NO) using mouse macrophage cells. Treatment with hesperidin suppressed production of $\mathrm{PGE}_{2}$, nitrogen dioxide $\left(\mathrm{NO}_{2}\right)$, and expression of iNOS protein. In the case of COX-2, hesperidin did not affect the protein levels expressed. Thus, hesperidin has been reported to be a COX-2 and iNOS inhibitor, which may explain its antiinflammatory and antitumorigenic efficacies in vivo. ${ }^{139}$ Furthermore, hesperetin and hesperidin in the concentration range $250-500 \mu \mathrm{M}$ have been shown to potently inhibit the LPS-induced expression of the COX-2 gene in RAW 264.7 cells, also 
demonstrating the antiinflammatory activity of these compounds. The ability of hesperetin and hesperidin to suppress COX-2 gene expression has been suggested to possibly be a consequence of their antioxidant activity. ${ }^{140}$

1.4.1.6 Antiadipogenic Activity Obesity is biologically characterized at the cellular level to be an increase in the number and size of adipocytes differentiated from fibroblastic preadipocytes in adipose tissue. It has been reported that hesperidin inhibits the formation of 3T3-L1 preadipocytes by $11.1 \%$. Apoptosis assays indicate that hesperidin increased apoptotic cells in a time- and concentration-dependent manner. Treatment of cells with hesperidin also decreased the mitochondrial membrane potential in a time- and dosedependent manner. The cell apoptosis/necrosis assay demonstrated that hesperidin increased the number of apoptotic cells but not necrotic cells. Hesperidin treatment of cells caused a significant time- and concentrationdependent increase in the CASP3 activity. Western blot analysis indicated that treatment of hesperidin also markedly downregulated poly ADP-ribose polymerase (PARP) and Bcl-2 proteins, and activated CASP3, Bax, and Bak proteins. These results indicate that hesperidin efficiently inhibits cell population growth and induction of apoptosis in 3T3-L1 preadipocytes. ${ }^{141}$ Furthermore, in the same in vitro, model hesperidin has been recently reported to inhibit intracellular triglyceride and glycerol-3-phosphate dehydrogenase (GPDH) activity by $40.2 \pm 3.2 \%$ and $37.9 \pm 4.6 \%$, respectively. ${ }^{142}$

1.4.1.7 Other Reported Activities Hesperidin and its aglycone, hesperetin, have been shown to have a very weak estrogenic effect, and its regular use can alleviate certain symptoms related with menopause and dysmenorrhea. ${ }^{143,144}$ For instance, in a controlled clinical study, 94 menopausal woman with hot flashes were given a daily formula for 1 month containing $900 \mathrm{mg}$ hesperidin, $300 \mathrm{mg}$ hesperidin methyl chalcone, and $1200 \mathrm{mf}$ vitamin C. After 1 month of treatment, the symptoms of hot flashes were completely relieved in $53 \%$ and reduced in $34 \%$ of the women. ${ }^{145}$

\subsubsection{Naringin and Naringenin}

Naringin ((+/-) 4',5,7-trihydroxyflavanone 7-rhamnoglucoside) $\mathrm{C}_{27} \mathrm{H}_{32} \mathrm{O}_{14}$, MW $580.53 \mathrm{~g} / \mathrm{mol}, \mathrm{X} \log \mathrm{P}$ value of -1 (Fig. 1.10), is a chiral flavanone-7-Oglycoside present in citrus fruits, tomatoes, cherries, oregano, beans, and cocoa. ${ }^{146-151}$ After consumption, the neohesperidose sugar moiety is rapidly cleaved off the parent compound in the gastrointestinal tract and liver to leave the aglycone bioflavonoid naringenin $((+/-)$ 4',5,7-trihydroxyflavanone) $\mathrm{C}_{15} \mathrm{H}_{12} \mathrm{O}_{5}$, MW $272.25 \mathrm{~g} / \mathrm{mol}, \mathrm{XLogP}$ value of 2.211 (Fig. 1.11). The ratio between the amount of naringenin and naringin varies among different food products. For instance, citrus fruits contain higher amounts of the glycoside naringin, while tomatoes have higher amounts of the aglycone naringenin. ${ }^{148}$ 


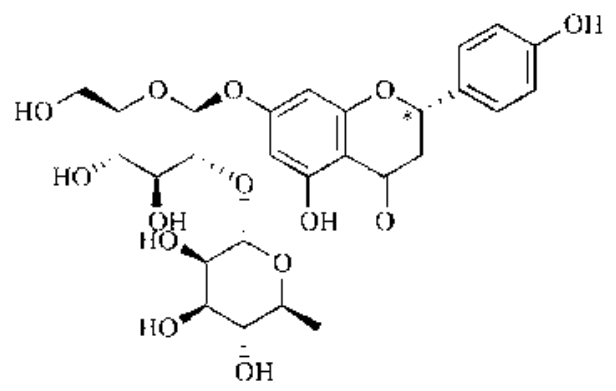

Figure 1.10. Structure of naringin. The asterisk $(*)$ denotes a chiral center.<smiles>O=C1CC(c2ccc(O)cc2)Oc2cc(O)ccc21</smiles>

Figure 1.11. Structures of naringenin. The asterisk (*) denotes a chiral center.

1.4.2.1 Antifungal, Antibacterial, and Antiviral Activity Naringin present in grapefruit ( $C$. paradise Macf., Rutaceae) seed and pulp ethanolic extracts has been related to have antibacterial and antifungal activity against multiple bacteria, fungi, and yeast strains. ${ }^{106,152}$ Naringin was assessed employing an in vitro agar assay and standard broth dilution susceptibility test, and it was observed that it exhibited the strongest antimicrobial effect against $S$. enteritidis (MIC of $2.06 \%$ extract concentration-m/V) and an MIC ranging from $4.13 \%$ to $16.5 \% \mathrm{~m} / \mathrm{V}$ for the other tested bacteria and yeasts. ${ }^{106}$ Similar results have been reported for naringin present in Argentine Tagetes (Asteraceae) ${ }^{153}$ and in Drynaria quercifolia. ${ }^{154}$

Naringenin isolated from ethanol extracts of propolis from four different regions of Turkey and Brazil exhibited to have MIC values ranging from 4 to $512 \mu \mathrm{g} / \mathrm{mL}$ for all the analyzed bacterial strains. Death was observed within 4 hours of incubation for Peptostreptococcus anaerobius, Peptostreptococcus micros, Lactobacillus acidophilus, and Actinomyces naeslundii, while 8 hours for Prevotella oralis and Prevotella melaninogenica and Porphyromonas gingivalis, 12 hours for Fusobacterium nucleatum, and 16 hours for Veillonella parvula. ${ }^{155}$ Similar results were found for naringenin-rich ethanol extracts of propolis having MIC values of $2 \mu \mathrm{g} / \mathrm{mL}$ for Streptococcus sobrinus and Enterococcus faecalis; $4 \mu \mathrm{g} / \mathrm{mL}$ for Micrococcus luteus, Candida albicans, and Candida krusei; $8 \mu \mathrm{g} / \mathrm{mL}$ for Streptococcus mutans, S. aureus, Staphylococcus epidermidis, and Enterobacter aerogenes; $16 \mu \mathrm{g} / \mathrm{mL}$ for Escherichia coli and Candida tropicalis; and $32 \mu \mathrm{g} / \mathrm{mL}$ for Salmonella typhimurium and 
Pseudomonas aeruginosa. ${ }^{156}$ Similar MIC values have been observed for naringenin isolated from the capitula of Helichrysum compactum. ${ }^{157}$ Naringenin has also been shown to have MIC $>20 \mu \mathrm{g} / \mathrm{mL}$ against $H$. pylori. However, neither naringenin nor other flavonoids and phenolic acids inhibited the urease activity of H. pylori. ${ }^{108}$

Naringenin has also been reported to have antiviral activity. For instance, naringenin exhibited an inhibitory effect on the replication of the NSV having a $50 \%$ inhibitory dose $\left(\mathrm{ID}_{50}\right)$ of $14.9 \mu \mathrm{g} / \mathrm{mL}$. However, its glycoside, naringin, did not have inhibitory activity. ${ }^{110}$ Similar results were observed for naringin, which was also ineffective on the replication of HSV-1, poliovirus type 1, Pf-3, and RSV in in vitro cell culture monolayers employing the technique of viral plaque reduction. ${ }^{83}$ Furthermore, naringenin has demonstrated activity against HSV-1 and type 2 (HSV-2) infected Vero cells in a virus-induced cytopathic effect (CPE) inhibitory assay, plaque reduction assay, and yield reduction assay. ${ }^{158}$ However, both naringin and naringenin are ineffective in inhibiting poliovirus replication. ${ }^{159}$

1.4.2.2 Antiinflammatory Activity Naringenin has been reported to have poor or no effect over different inflammatory mediators in vitro. For instance, naringenin was ineffective in inhibiting endothelial adhesion molecule expression or in attenuating expression of E-selectin and ICAM-1, VCAM-1, and TNF- $\alpha$-induced adhesion molecule expression in human aortic endothelial cells. ${ }^{160}$ In another study, naringenin also exhibited virtually no effects on cytokines, metabolic activity, or on the number of cells in the studied cell populations of stimulated human peripheral blood mononuclear cells (PBMCs) by LPS. ${ }^{161}$ Furthermore, the lack of ability of naringenin to inhibit the activity of NOS-2 has been reported; however, the induction of NOS-2 protein in LPStreated $\mathbf{J} 774.2$ cell was evident by Western blotting techniques. ${ }^{162}$

However, naringin has been reported to regulate certain inflammatory mediators and to possess antiinflammatory activity. Naringin (10, 30, and $60 \mathrm{mg} / \mathrm{kg}$ intraperitoneal [i.p.]) dose dependently suppressed LPS-induced production of TNF- $\alpha$ in mice. To further examine the mechanism by which naringin suppresses LPS-induced endotoxin shock, an in vitro model, RAW 264.7 mouse macrophage cells, was utilized. Naringin $(1 \mathrm{mM})$ suppressed LPSinduced production of $\mathrm{NO}$ and the expression of inflammatory gene products such as iNOS, TNF- $\alpha$, inducible cyclooxygenase (COX-2), and IL-6 as determined by RT-PCR assay. Naringin was also found to have blocked the LPSinduced transcriptional activity of NF- $\kappa \mathrm{B}$ in electrophoretic mobility shift assay and reporter assay. These findings suggest that suppression of the LPSinduced mortality and production of NO by naringin is due to inhibition of the activation of NF- $\kappa$ B. ${ }^{163}$

Similarly, a separate study assessed the effect of naringin in an endotoxin shock model based on Salmonella infection. Intraperitoneal (i.p.) infection with $10 \mathrm{cfu}$ S. typhimurium aroA caused lethal shock in LPS-responder but not in LPS-nonresponder mice. Administration of $1 \mathrm{mg}$ naringin 3 hours 
before infection resulted in protection from lethal shock, similar to LPSnonresponder mice. The protective effect of naringin was time- and dose dependent. Treatment with naringin resulted not only in a significant decrease in bacterial numbers in spleens and in livers, but also in a decrease in plasma LPS levels. In addition, naringin markedly suppressed TNF- $\alpha$ and normalized the activated states of blood coagulation factors such as prothrombin time, fibrinogen concentration, and platelet numbers caused by infection. ${ }^{164}$

1.4.2.3 Antioxidant Activity Different in vitro chemical and biological assays have reported that naringin and naringenin have considerable antioxidant properties. For instance, naringin has been reported to scavenge the DPPH, 2,2'-azinobis-(3-ethyl-benzothiazoline-6-sulfonic acid) (ABTS) and nitric oxide (NO) radicals in vitro in a concentration-dependent manner. ${ }^{165}$ Furthermore, naringin and naringenin have been assessed in the beta-carotenelinoleic acid, DPPH, superoxide, and hamster LDL in vitro models to measure their antioxidant activity. Using the beta-carotene-linoleate model, naringin $(10 \mu \mathrm{M})$ and naringenin $(10 \mu \mathrm{M})$ exhibited an $8 \%$ and $9 \%$ inhibition, respectively, whereas both compounds demonstrated negative free radical scavenging activity using the DPPH method and a $25 \%$ and $30 \%$ inhibition of superoxide radicals for naringin and naringenin, respectively. Naringin and naringenin increased the lag time of LDL oxidation to 150 minutes (a 32\% increase from baseline levels). Thus, indicating that both compounds have significant in vitro antioxidant properties. ${ }^{166}$ Furthermore, naringin has been reported to have a positive effect in iron-induced oxidative stress and in a variety of cellular processes like respiration and DNA synthesis. For this, HepG2 cells were treated with $0.5,1.0,2.5$, and $5.0 \mathrm{mM}$ naringin 1 hour before exposure to $0.1,0.25,0.5$, and $1.0 \mathrm{mM}$ ferric iron. Pretreatment of HepG2 cells with naringin resulted in inhibition of lipid peroxidation, arrested the ironinduced depletion in the GSH concentration, and increased various antioxidant enzymes like glutathione peroxidase (GSHPx), CAT, and SOD. ${ }^{167}$

Naringin has also demonstrated antioxidant properties in different in vivo animal models. A comparison study between grapefruit juice and naringin reported that the total antioxidant activity of a quantity of red grapefruit juice was higher than that of naringin. Animals received a cholesterol-rich diet and after administration of naringin (0.46-0.92 mg p.o.) or red grapefruit juice $(1.2 \mathrm{~mL})$, it was observed that diets supplemented with red grapefruit juice and, to a lesser degree, with naringin improved the plasma lipid levels and increased the plasma antioxidant activity. ${ }^{168}$

1.4.2.4 Anticancer Activity Naringin and naringenin have been reported to have anticancer activities. For instance, naringenin has been reported to induce cytotoxicity in cell lines derived from cancer of the breast (MCF-7, MDA-MB-231), stomach (KATOIII, MKN-7), liver (HepG2, Hep3B, and Huh7), cervix (Hela, Hela-TG), pancreas (PK-1), and colon (Caco-2), as well as leukemia (HL60, NALM-6, Jurkat, and U937). Naringenin-induced cytotoxicity was low in Caco-2 and high in leukemia cells compared to other 
cell lines. Naringenin dose dependently induced apoptosis, with hypodiploid cells detected in both Caco-2 and HL60 by flow cytometric analysis. ${ }^{169}$ Furthermore, naringenin at concentrations higher than $0.71 \mathrm{mM}$ has been reported to inhibit cell proliferation of HT29 colon cancer cells, ${ }^{170}$ while naringin has been reported to induce cytotoxicity via apoptosis in mouse leukemia P388 cells and to slightly increase the activities of the antioxidant enzymes, CAT, and GSHPx in these cells. ${ }^{171}$

Naringin and naringenin have also been assessed for its effects on proliferation and growth of a human breast carcinoma cell line, MDA-MB-435. The concentration at which cell proliferation was inhibited by $50 \%\left(\mathrm{IC}_{50}\right)$ was around $20 \mu \mathrm{g} / \mathrm{mL}$ for naringin and naringenin with low cytotoxicity ( $>500 \mu \mathrm{g} /$ $\mathrm{mL}$ for $50 \%$ cell death). ${ }^{88}$ Two possible mechanisms that could modulate breast tumor growth have been proposed, one via inhibition of aromatase (CYP19) and the other via interaction with the estrogen receptor (ER). Multiple in vitro studies confirmed that naringin and naringenin act as aromatase inhibitors potentially reducing tumor growth. It is thought that in the in vivo situation, breast epithelial (tumor) cells communicate with surrounding connective tissue by means of cytokines, prostaglandins, and estradiol forming a complex feedback mechanism. It has been reported that naringenin affects MCF-7 proliferation with an $\mathrm{EC}_{50}$ value of $287 \mathrm{nM}$ and acts as an aromatase inhibitor with an $\mathrm{IC}_{50}$ value of $2.2 \mu \mathrm{M}$. These results show that naringenin can induce cell proliferation or inhibit aromatase in the same concentration range $(1-10 \mu \mathrm{M}){ }^{172}$ The second proposed mechanism is related to the ER, and it has been observed that naringenin exerts an antiproliferative effect only in the presence of $\mathrm{ER} \alpha$ or $\mathrm{ER} \beta$. Moreover, naringenin stimulation induces the activation of $\mathrm{p} 38$ /MAPK leading to the proapoptotic CASP3 activation and to the poly(ADP-ribose) polymerase cleavage in selected cancer cell lines. Notably, naringenin shows an antiestrogenic effect only in ER $\alpha$-containing cells, whereas in ER $\beta$-containing cells, naringenin mimics the 17beta-estradiol effects. ${ }^{173}$ Nevertheless, naringenin-mediated growth arrest in MCF-7 breast cancer cells has also been observed. Naringenin was found to inhibit the activity of phosphoinositide 3-kinase (PI3K), a key regulator of insulin-induced GLUT4 translocation, as shown by impaired phosphorylation of the downstream signaling molecule Akt. Naringenin also inhibited the phosphorylation of p44/p42 MAPK. Inhibition of the MAPK pathway with PD98059, a MAPK kinase inhibitor, reduced insulin-stimulated glucose uptake by approximately $60 \%$. The MAPK pathway therefore appears to contribute significantly to insulin-stimulated glucose uptake in breast cancer cells. ${ }^{174}$

In the case of human prostate cancer cells (PC3) stably transfected with activator protein 1 (AP-1) luciferase reporter gene, the maximum AP-1 luciferase induction is of about threefold over control after treatment with naringenin $(20 \mu \mathrm{M})$. At higher concentrations, naringenin demonstrated inhibition of AP-1 activity. The MTS assay for cell viability at 24 hours demonstrated that even at a very high concentration $(500 \mu \mathrm{M})$, cell death was minimal for naringenin. Furthermore, induction of phospho-C-Jun N-terminal kinase (JNK) and phospho-ERK activity was observed after a 2-hour incubation of 
PC3-AP-1 cells with naringenin. However, no induction of phospho-p38 activity was observed. Furthermore, pretreating the cells with specific inhibitors of JNK reduced the AP-1 luciferase activity that was induced by naringenin, while pretreatment with MAPK (MEK) inhibitor did not affect the AP-1 luciferase activity. ${ }^{175}$ It was also observed that naringenin induced apoptosis of human promyeloleukemia HL60 cells by markedly promoting the activation of CASP3, and slightly promoting the activation of caspase-9, but with no observed effect on caspase- $8{ }^{176}$ The apoptosis-induced mechanism of naringenin has also been linked with the activation of NF- $\kappa \mathrm{B}$ and the degradation of $\mathrm{I} \kappa \mathrm{B} \alpha$, which has been observed in human promyeloleukemia HL60 cells, ${ }^{176}$ in human colon carcinoma HCT116 cells, and in human liver carcinoma HepG2 cells. ${ }^{177}$

Neoangiogenesis is required for tumor development and progression. Many solid tumors induce vascular proliferation by production of angiogenic factors, prominently vascular endothelial growth factor (VEGF). It has been reported that naringin has a significant inhibitory activity against VEGF at $0.1 \mu \mathrm{M}$ in MDA-MB-231 human breast cancer cells and that glioma cells were similarly sensitive, with U343 more active than U118. Inhibition of VEGF release by naringin in these models of neoplastic cells suggests a novel mechanism for mammary cancer prevention. ${ }^{178}$

Animal models have also demonstrated that grapefruit juice as well as the isolated citrus compound naringin can protect against AOM-induced ACF by suppressing proliferation and elevating apoptosis through antiinflammatory activities. Grapefruit juice suppressed aberrant crypt formation and high multiplicity ACF (HMACF) formation and expansion of the proliferative zone that occurs in the AOM-injected rats consuming the control diet. Grapefruit juice also suppressed elevation of both iNOS and COX-2 levels observed in AOM-injected rats consuming the control diet. Naringin suppressed iNOS levels in AOM-injected rats; no effect was observed with respect to COX-2 levels. Thus, lower levels of iNOS and COX-2 are associated with suppression of proliferation and upregulation of apoptosis, which may have contributed to a decrease in the number of HMACF in rats provided with naringenin. These results suggest that consumption of grapefruit juice or naringin may help to suppress colon cancer development. ${ }^{179}$ Similar inhibition in tumor growth and formation in sarcoma S-180-implanted mice have been reported for naringenin. ${ }^{169}$

1.4.2.5 Cyclooxygenase-1 and -2 Inhibitory Activity Naringenin has been assessed for its effects on nitric oxide (NO) and $\mathrm{PGE}_{2}$ production induced by LPS in the macrophage cell line J774A.1. Naringenin $(0.5-50.0 \mu \mathrm{M})$ was observed to be a significant inhibitor of NO production, and this effect was concentration dependent and significant at both 5 and $50 \mu \mathrm{M}$. A similar pattern was observed with the inhibitory effect of naringenin on LPS-induced $\mathrm{PGE}_{2}$ release and COX-2 expression. Naringenin markedly decreased $\mathrm{PGE}_{2}$ release and COX-2 expression in a concentration-dependent manner. Thus, narin- 
genin inhibits iNOS and COX-2 expression and may be one of the important mechanisms responsible for their antiinflammatory effects. ${ }^{180}$

1.4.2.6 Antiadipogenic Activity A recent study has looked at the activity of naringin and naringenin and other flavonoids on preadipocyte cell population growth. The results demonstrated that the inhibition of naringin and naringenin on 3T3-L1 preadipocytes was 5.6\% and $28.3 \%$, respectively. Apoptosis assays demonstrated that naringin and naringenin increased apoptotic cells in a time- and concentration-dependent manner. Treatment of cells with naringin and naringenin also decreased the mitochondrial membrane potential in a time and dose-dependent manner. The cell apoptosis/necrosis assay demonstrated that both naringin and naringenin increased the number of apoptotic cells but not necrotic cells. Naringin and naringenin treatment of cells caused a significant time- and dose-dependent increase in the CASP3 activity. Western blot analysis indicated that treatment with both naringin and naringenin also markedly downregulated PARP and Bcl-2 proteins, and activated CASP3, Bax, and Bak proteins. These results suggest that the glycoside naringin and the aglycone naringenin efficiently inhibit cell population growth and induction of apoptosis in 3T3-L1 preadipocytes. ${ }^{141}$ Furthermore, in this same in vitro model, naringin and naringenin have been recently reported to inhibit intracellular triglyceride by $41.3 \pm 8.4 \%$ and $39.4 \pm 7.8 \%$, respectively, and also to inhibit GPDH activity by $39.4 \pm 5.6 \%$ and $35.7 \pm 1.4 \%$, respectively. ${ }^{142}$

1.4.2.7 Cardioprotective Effects Naringin (10, 20, and $40 \mathrm{mg} / \mathrm{kg}$, administered orally for 56 days) has been reported to decrease heart weight, blood glucose, serum uric acid, serum iron, levels of total proteins, and iron binding capacity, as well as to increase $\mathrm{Na}(+) / \mathrm{K}(+)$ ATPase and to decrease the activities of $\mathrm{Ca}(2+)$ and $\mathrm{Mg}(2+)$ ATPase in the heart and the levels of glycoproteins in serum and in the heart in an isoproterenol $(85 \mathrm{mg} / \mathrm{kg} \mathrm{sc}$ ) (ISO)-induced myocardial infarction (MI) animal model. ${ }^{165}$ Similar results have been observed for naringin reducing the levels of cardiac troponin $\mathrm{T}(\mathrm{cTnT})$, lactate dehydrogenase (LDH)-isoenzymes 1 and 2, cardiac marker enzymes, electrocardiographic (ECG) patterns and lysosomal hydrolases. ${ }^{181}$

1.4.2.8 Effect on Cytochrome P450 Naringin and naringenin are the main flavanones present in grapefruit juice. These compounds have been shown to markedly augment the oral bioavailability of several drugs. ${ }^{146}$ This effect was originally based on an unexpected observation from an interaction study between the dihydropyridine calcium channel antagonist, felodipine, and ethanol in which grapefruit juice was used to mask the taste of the ethanol. ${ }^{182}$ Naringenin has been reported to competitively inhibit CYP3A4 altering the bioavailability of felodipine ${ }^{183}$ most dihydropyridines, terfenadine, saquinavir ${ }^{184}$ cyclosporin, midazolam, triazolam, quinine, ${ }^{185}$ verapamil ${ }^{186}$ and one of 
the verapamil metabolites, norverapamil ${ }^{187}$ and this interaction may also occur with lovastatin, cisapride, and astemizole. ${ }^{188,189}$

Grapefruit juice contains a variety of flavonoid molecules, such as naringin, naringenin, quercetin, and kaempferol, and some nonflavonoid molecules such as $6^{\prime}, 7^{\prime}$-dihydroxybergamottin, which are known to inhibit CYP3A4 activity in vitro. ${ }^{190}$ These polyphenolic compounds are electron-rich molecules and, therefore, are likely substrates for CYP3A4 and may inhibit the enzyme. ${ }^{190}$ These molecules are known to interfere with intestinal CYP3A4 and hepatic CYP2A6, thereby lowering the biotransformation of several drugs and increasing their bioavailability ${ }^{191}$ Earlier efforts to identify the inhibitory substance(s) present in grapefruit juice largely focused on naringin and quercetin. However, when administered to humans, both compounds failed to reproduce the inhibition of dihydropyridine metabolism caused by grapefruit juice. ${ }^{192,193}$ Edwards and Bernier ${ }^{194}$ have suggested that naringin and naringenin are not the primary inhibitory compounds in grapefruit juice, although results from rat and human liver microsomes demonstrate that naringenin and other flavonoids in grapefruit juice can inhibit the metabolism of dihydropyridine calcium antagonists. ${ }^{195,196}$ In the continued quest to verify and identify the active inhibitor in grapefruit juice, $6^{\prime}, 7^{\prime}$-dihydroxybergamottin, a furanocoumarin, was identified as a potent inhibitor of CYP3A4 activity. ${ }^{197}$ This study was followed by another study that confirmed the presence of $6^{\prime}, 7^{\prime}$-dihydroxybergamottin as a major substance in grapefruit juice being responsible for enhanced oral availability of CYP3A4 substrates, although other furanocoumarins probably also contribute to this phenomena. ${ }^{198}$ These results have been corroborated by others ${ }^{199}$ that reported similar findings of altered bioavailability. It has been suggested that hydrophilic components other than flavonoids, probably coumarin derivatives, are also responsible for the inhibitory effect of grapefruit juice. In another recent study, it was found that naringin alone was ineffective in causing the inhibition of the metabolism of 1,2-benzopyrone (coumarin) in humans, thereby concluding that the inhibitory effect of grapefruit juice may be modulated by naringenin. ${ }^{191}$ In view of the existing literature, it is apparent that the inhibition of first-pass metabolism by grapefruit juice probably involves the flavonoid naringenin and also furanocoumarins. Recent reviews on drug interactions with grapefruit juice are available elsewhere. ${ }^{200-202}$ Concern regarding the mechanism of inhibition of CYP3A enzymes by grapefruit juice has now centered on protein expression studies. In a recent study, a selective $62 \%$ downregulation of CYP3A4 protein levels in small intestine epithelia (enterocytes) with no corresponding change in CYP3A4 mRNA levels was reported. ${ }^{203}$ In contrast, grapefruit juice did not alter hepatic CYP3A4 activity, colon levels of CYP3A5, or small bowel concentrations of P-glycoprotein, villin, CYP1A1, and CYP2D6. In another study, it was demonstrated that grapefruit juice induced a two- to fivefold increase in the ability of the P-glycoprotein pump to transport drugs such as vinblastine, cyclosporin, losartan, digoxin, and fexofenadine across intestinal cell monolayers in vitro. ${ }^{204}$ However, drugs such as nifedipine and felodipine were not transported by P-glycoprotein in these cells, and their passage through the monolayer was unaffected by grapefruit 
juice since these drugs are not P-glycoprotein substrates. Orange juice is also known to inhibit the activity of CYP3A enzymes; however, there is a large difference between grapefruit and orange juice in their enzyme inhibition potencies. The difference in potency may be accountable in part to lack of detectable naringin ${ }^{205}$ and $6^{\prime}, 7^{\prime}$-dihydroxybergamottin ${ }^{197}$ in orange juice. Perhaps this may partly explain why orange juice did not affect the bioavailability of orally administered nifedipine ${ }^{205}$ or pranidipine, ${ }^{206}$ whereas grapefruit juice significantly increased their bioavailability. Nevertheless, red wine, which also contains a complex mixture of flavonoids and other polyphenolic compounds, inhibits CYP3A4 activity in vitro. ${ }^{190}$ Interestingly, white wine and its components do not apparently inhibit CYP3A4 activity. ${ }^{190}$

1.4.2.9 Other Reported Activities Naringin has also been reported to have antigenotoxic properties. Naringin was assessed in an in vitro biological model: bleomycin-induced genomic damage of cultured V79 cells. Exposure of V79 cells to bleomycin $(50 \mu \mathrm{g} / \mathrm{mL})$ induced a concentration-dependent elevation in the frequency of binucleate cells bearing micronuclei (MNBNC) and a maximum number of MNBNCs. Treatment of cells with $1 \mathrm{mM}$ naringin before exposure to different concentrations of bleomycin arrested the bleomycin-induced decline in cell survival accompanied by a significant reduction in the frequency of micronuclei when compared with bleomycin treatment alone. The cell survival and micronuclei induction were found to be inversely correlated. The repair kinetics of DNA damage induced by bleomycin was evaluated by exposing the cells to $10 \mu \mathrm{g} / \mathrm{mL}$ bleomycin using single-cell gel electrophoresis. Treatment of V79 cells with bleomycin resulted in a continuous increase in DNA damage up to 6 hours postbleomycin treatment as evident by the migration of greater amounts of DNA into the tails (\% tail DNA) of the comets and a subsequent increase in olive tail moment (OTM), an index of DNA damage. Treatment of V79 cells with $1 \mathrm{mM}$ naringin reduced bleomycin-induced DNA damage and accelerated DNA repair as indicated by a reduction in percent tail DNA and OTM with an increasing assessment time. A maximum reduction in the DNA damage was observed at 6 hours post bleomycin treatment, where it was five times lower than bleomycin alone. $^{207}$

Other reported effects of naringin include protection against radiationinduced chromosome damage. For this, naringin extracted from the ethyl acetate fraction of Aphanamixis polystachya was investigated on the radiationinduced chromosome damage in the bone marrow cells of Swiss albino mice exposed to various doses of gamma radiation. The mice were divided into two groups: One group was exposed to $0,1,2,3,4$, or $5 \mathrm{~Gy}$ of gamma radiation, while another group received $7.5-\mathrm{mg} / \mathrm{kg} \mathrm{BW}$ of the ethyl acetate fraction of $A$. polystachya 1 hour before exposure to $0,1,2,3,4$, or 5 Gy of gamma radiation. Various asymmetrical chromosome aberrations were studied in the bone marrow cells of mice at 12, 24, or 48 hours postirradiation. Irradiation of mice to various doses of gamma radiation caused a dose-dependent elevation in the frequency of aberrant cells and chromosome aberrations like chromatid 
breaks, chromosome breaks, dicentrics, acentric fragments, and total aberrations at all the postirradiation times studied. The maximum asymmetrical aberrations were scored at 24 hours postirradiation except chromatid breaks that were highest at 12 hours postirradiation. A maximum number of polyploid and severely damaged cells (SDCs) were recorded at 24 hours postirradiation in the SPS plus irradiation group. Treatment of mice with $7.5 \mathrm{mg} / \mathrm{kg} \mathrm{BW}$ of the naringin-rich ethyl acetate fraction of $A$. polystachya before exposure to 1-5 Gy of whole body gamma radiation significantly reduced the frequencies of aberrant cells and chromosomal aberrations like acentric fragments, chromatid and chromosome breaks, centric rings, dicentrics, and total aberrations at all postirradiation scoring times. It can be observed from this study that the naringin-rich ethyl acetate fraction of $A$. polystachya protects mouse bone marrow cells against radiation-induced chromosomal aberrations, and this reduction in radiation-induced chromosome damage may be due to free radical scavenging and reduction in lipid peroxidation. The radioprotection caused by the naringin-rich ethyl acetate fraction of $A$. polystachya is comparable to the protection demonstrated by naringin. ${ }^{208}$

\subsubsection{Eriocitrin and Eriodictyol}

Eriocitrin ((+/-) -5,7,3',4-tetrahydroxyflavanone 7-O-ruinoside) $\mathrm{C}_{27} \mathrm{H}_{32} \mathrm{O}_{15}$, MW $596.53 \mathrm{~g} / \mathrm{mol}$, XLogP value of -1.4 (Fig. 1.12) is a chiral flavanone-7-Oglycoside present in lemons, tamarinds, and other citrus fruits, as well as in mint, oregano, fennel thyme, and rose hip. ${ }^{52,147,209-213}$ After consumption, the neohesperidose sugar moiety is rapidly cleaved off the parent compound in the gastrointestinal tract and liver to leave the aglycone bioflavonoid eriodictyol ((+/-)-5,7,3',4'-tetrahydroxyflavanone) $\mathrm{C}_{15} \mathrm{H}_{12} \mathrm{O}_{6}$, MW $288.25 \mathrm{~g} / \mathrm{mol}$, XLogP value of 1.837 (Fig. 1.13).

1.4.3.1 Antibacterial Activity Eriocitrin extracted from peppermint (Mentha piperita L.) leaves has been demonstrated to have antimicrobial

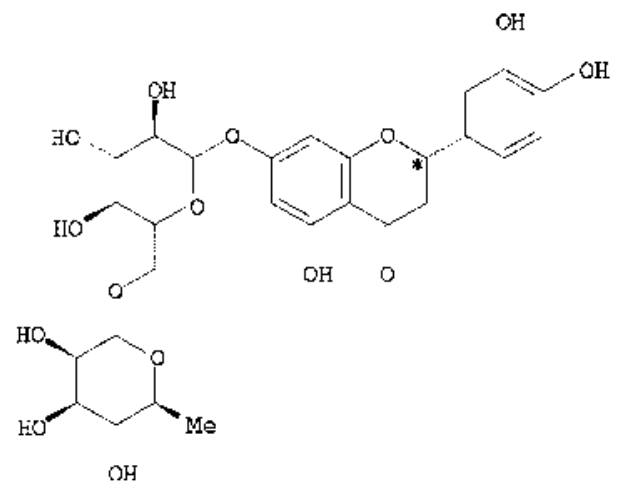

Figure 1.12. Structure of eriocitrin. The asterisk (*) denotes a chiral center. 


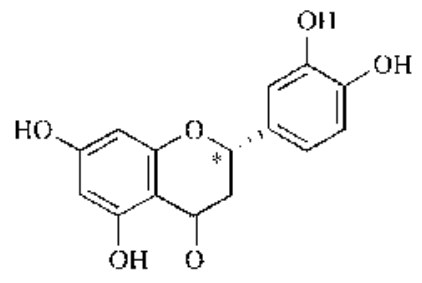

Figure 1.13. Structures of eriodictyol. The asterisk (*) denotes a chiral center.

activities. ${ }^{214}$ Furthermore, eriodictyol extracted from the leaves of Rhus retinorrhoea Steud, ex Olive has exhibited moderate antimalarial activity with an $\mathrm{IC}_{50}$ of $0.98 \mu \mathrm{g} / \mathrm{mL}$ against Plasmodium falciparum (W2 clone) and weak activity against $P$. falciparum (D6 clone) with an $\mathrm{IC}_{50}$ of $2.8 \mu \mathrm{g} / \mathrm{mL}$, with no cytotoxic effects. ${ }^{215}$ However, eriodictyol isolated from Gleditsia sinensis Lam. spines demonstrated a lack of activity against Xanthomonas vesicatoria and Bacillus subtilis. ${ }^{216}$

1.4.3.2 Antiinflammatory Activity Eriodictyol extracted from Thymus broussonetii Boiss (Labiatae) leaves, a herbal drug used in Moroccan traditional medicine, has been assessed using the croton oil ear test in mice and reported significant antiinflammatory properties. ${ }^{217}$ Furthermore, pretreatment of RAW 264.7 with eriodictyol inhibited TNF- $\alpha$ release in LPS-stimulated macrophages. The potency of eriodictyol in inhibiting cytokine production was reported with an $\mathrm{IC}_{50}$ of less than $10 \mu \mathrm{M}$ for $\mathrm{TNF}-\alpha$ release. It was also observed that pretreatment of cells with eriodictyol decreased I $\kappa \mathrm{B}-\alpha$ phosphorylation and reduced the levels of $\mathrm{I} \kappa \mathrm{B}-\alpha{ }^{218}$

1.4.3.3 Antioxidant Activity Eriocitrin and eriodictyol isolated from lemon (Citrus limon) juice exhibited a potent radical scavenging activity for DPPH and superoxide. Eriocitrin and eriodictyol were found to significantly suppress the expression of ICAM-1 at $10 \mu \mathrm{M}$ in human umbilical vein endothelial cells (HUVECs) induced by TNF- $\alpha{ }^{219}$ Eriocitrin obtained from peppermint leaves (Menthae $\times$ piperitae folium) (total eriocitrin $38 \%$ ) exhibited a strong antiradical activity (determined as DPPH* scavenging features). Eriocitrin also exhibited a strong anti- $\mathrm{H}_{2} \mathrm{O}_{2}$ activity. ${ }^{220}$ Similarly, eriocitrin extracted from different Mentha species, varieties, hybrids, and cultivars was identified as the dominant radical scavenger in these extracts in an online high performance liquid chromatography-1,1-diphenyl-2-picrylhydrazyl (HPLC-DPPH*) method. ${ }^{210}$ Furthermore, eriocitrin was reported to play a role as antioxidant in vivo in streptozotocin-induced diabetic rats. Diabetic rats were provided a diet that contained $0.2 \%$ eriocitrin. After the 28 -day feeding period, the concentration of the TBARS in the serum, liver, and kidney of diabetic rats administered eriocitrin significantly decreased as compared 
with that of the diabetic group. The levels of 8-hydroxydeoxyguanosine, which is exchanged from deoxyguanosine owing to oxidative stress, in the urine of diabetic rats administered eriocitrin significantly decreased as compared with that of the diabetic rat group. Eriocitrin also suppressed oxidative stress in the diabetic rats. $^{221}$

Eriodictyol isolated from the aerial parts from Eysenhardtia subcoriacea was assessed using an antioxidant activity assay-guided chemical analysis, using a rat pancreas homogenate model. The isolated eriodictyol demonstrated moderate radical scavenging properties against DPPH radical ${ }^{222,223}$ and reduced the GSH levels in rat pancreatic homogenate. ${ }^{222}$ Furthermore, eriodictyol was assessed for its protective role against UV-induced apoptosis of human keratinocytes, the principal cell type of epidermis. The results demonstrated that eriodictyol had a positive effect on cell proliferation of human $\mathrm{HaCaT}$ keratinocytes. Treatment with eriodictyol, in particular, resulted in significant suppression of cell death induced by UV light, a major skindamaging agent. It was also observed that eriodictyol treatment apparently reduced the percentage of apoptotic cells and the cleavage of poly(ADPribose) polymerase, concomitant with the repression of CASP3 activation and reactive oxygen species (ROS) generation. The antiapoptotic and antioxidant effects of eriodictyol were also confirmed in UV-induced cell death of normal human epidermal keratinocyte (NHEK) cells, suggesting that eriodictyol can be used to protect keratinocytes from UV-induced damage, implying the presence of a complex SAR in the differential apoptosis-modulating activities of eriodictyol and similar flavonoid compounds. ${ }^{224}$

1.4.3.4 Anticancer Activity Eriodictyol extracted from lemon fruit $(C$. limon Burm. f.) altered the DNA fragmentation of HL60 cells when analyzed by flow cytometry. An apoptotic DNA ladder and chromatin condensation were observed in HL60 cells when treated with eriodictyol. ${ }^{225}$ Eriodictyol was also assessed for its protective role against UV-induced apoptosis of human keratinocytes, the principal cell type of the epidermis. The results demonstrated that eriodictyol had a positive effect on cell proliferation of human $\mathrm{HaCaT}$ keratinocytes. Treatment with eriodictyol in particular resulted in significant suppression of cell death induced by UV light, a major skin-damaging agent. Eriodictyol treatment apparently reduced the percentage of apoptotic cells and the cleavage of poly(ADP-ribose) polymerase, concomitant with the repression of CASP3 activation and ROS generation. The antiapoptotic and antioxidant effects of eriodictyol were also confirmed in UV-induced cell death of NHEK cells. ${ }^{224}$ Eriodictyol also protected L-929 cells from TNFinduced cell death. The magnitude of protection and potentiation by eriodictyol was concentration dependent, and these effects were not altered when eriodictyol was added as much as 2 hours after TNF treatment. ${ }^{226}$ Eriodictyol possess antiproliferative activities against several tumor and normal human cell lines. Eriodictyol has $\mathrm{IC}_{50}$ of $12,10,8.3$, and $6.2 \mu \mathrm{M}$ in human lung carcinoma (A549), melanin pigment producing mouse melanoma (B16 melanoma 
4A5), human T-cell leukemia (CCRF-HSB-2), and metastasized lymph node (TGBC11TKB), respectively. ${ }^{227}$

1.4.3.5 Cyclooxygenase-1 and-2 Inhibitory Activity Eriodictyol extracted from the methanol fraction of the stem bark of Populus davidiana demonstrated moderate inhibition against COX-1 only and exhibited suppressive effects on $\mathrm{XO}^{228}$

1.4.3.6 Other Reported Activities Eriodictyol also been reported to have antimutagenic activities in a model induced by tert-butyl hydroperoxide (BHP) or cumene hydroperoxide (CHP) in S. typhimurium TA102 $\left(\mathrm{ID}_{50}<\right.$ $1 \mu \mathrm{mol}$ per plate). These effects correlated with the radical scavenging activities of eriodictyol against peroxyl radicals generated from 2,2'-azo-bis(2amidinopropane)dihydrochloride (AAPH) as measured in the hemolysis test and confirmed that, in general, eriodictyol is an effective radical scavenger. From these results, it was concluded that in the Salmonella/reversion assay with strain TA102, the antimutagenic activities of eriodictyol against the peroxide mutagens $\mathrm{CHP}$ and BHP are mainly caused by radical scavenging effects. $^{223}$

\subsubsection{Phloretin}

Phloretin [3-(4-hydroxyphenyl)-1-(2,4,6-trihydroxyphenyl)propan-1-one] $\mathrm{C}_{15} \mathrm{H}_{14} \mathrm{O}_{5}$, MW $274.3 \mathrm{~g} / \mathrm{mol}$ is a hydrophobic, polyphenolic compound (XLogP 2.6). Phloretin's structure varies from the stilbenoid structure of pterostilbene and phloretin as it exists as a dihydrochalcone (Fig. 1.14). A dihydrochalcone is defined by the presence of two benzenoid rings connected by three carbons.

Phloretin has been identified in apples and in other natural sources including Pieris japonica, Kalmia latifolia, Hoveniae lignum, and Loiseleuria procumbens. ${ }^{229-234}$ Phloridzin is the glycoside of phloretin (phloretin-2'-glucose) (Fig. 1.15). Phloridzin has been identified in apples, strawberries, and in several other plants including P. japonica and Lithocarpus pachyphyllus. ${ }^{233,235-237}$ After consumption, it has been suggested that the glucose sugar moiety of phloridzin is rapidly cleaved off the parent compound in the gastrointestinal tract and liver to leave the aglycone, phloretin. ${ }^{235}$ It has been suggested that the aglycone

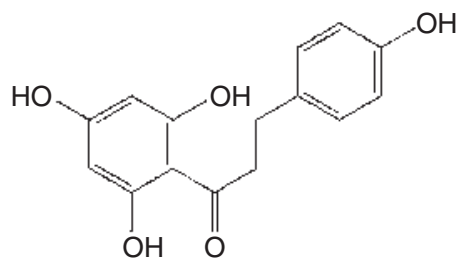

Figure 1.14. Chemical structure of phloretin. 


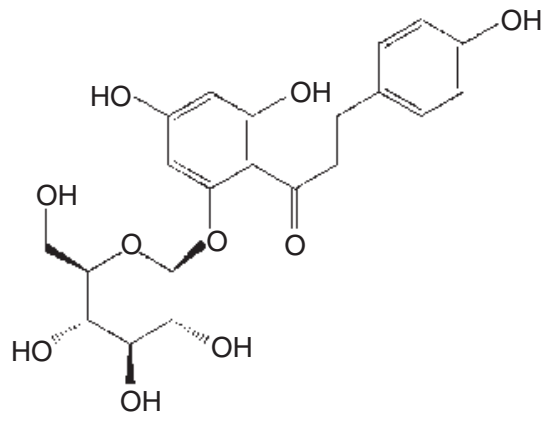

Figure 1.15. Chemical structure of phloridzin.

form of polyphenols is pharmacologically a more active species than the glycoside form. ${ }^{238}$

Phloretin and phloridzin show structural similarity to other dihydrochalcones specifically those isolated from rooibos (Aspalathus linearis), a broomlike shrub commonly used as a caffeine-free tea in South Africa. ${ }^{239}$ Besides a strong antioxidant capacity, rooibos has shown numerous bioactivities including antimutagenic, ${ }^{240}$ hepatoprotective, ${ }^{241}$ and hypoglycemic properties. ${ }^{242}$ Two C-linked dihydrochalcone glucosides have been associated with the antioxidant properties of rooibos including aspalathin and nothofagin. ${ }^{243,244}$ Aspalathin itself has extensive antioxidant abilities, abilities to modulate blood sugars, and hepatoprotective properties. ${ }^{242,244,245}$ Nothofagin demonstrates slightly less potent antioxidant activities. ${ }^{243}$

Research to characterize the pharmacological activities of both phloretin and phloridzin has shown these compounds reduce oxidative stress, induce apoptosis, and alter glucose transport. ${ }^{235,246-256}$ Most importantly, apples, which contain large amounts of phloretin and phloridzin, have been correlated with numerous health benefits including reduced risk of cardiovascular disease, asthma, some cancers, and diabetes. ${ }^{257}$ A summary of activities of phloretin and phloridzin follows below.

1.4.4.1 Glucose Transport Phloretin and phloridzin both are well-known inhibitors of glucose transport. Phloridzin is an inhibitor of SGLT1 and SGLT2, sodium-linked active glucose transporters found in the small intestine and in the proximal tubule of nephrons. ${ }^{235,256}$ Phloretin is an inhibitor of GLUT2, a facilitated glucose transporter found in the liver, pancreatic $\beta$-cells, and the basolateral surface of kidney and intestinal epithelia. ${ }^{255,256}$ GLUT2 has also been shown to traffic to the apical membrane of intestinal epithelium in response to high glucose concentrations during assimilation of a meal. ${ }^{258,259}$ This apical GLUT2 is a major pathway of sugar absorption. ${ }^{260}$ Polyphenols from strawberries and apples, specifically quercetin, have reported activities in inhibiting this intestinal GLUT2 in Caco-2 cells. ${ }^{261}$ It is thought that since 
numerous polyphenols are poorly bioavailable and will attain their highest concentrations in the gastrointestinal tract lumen, they may be promising agents for obesity treatment. ${ }^{260}$

1.4.4.2 Antioxidant Activity Phloretin possesses potent antioxidant activity that has been attributed to its unique dihydrochalcone structure. Phloretin has been shown to be a potent peroxynitrite, hydroxyl radical, and DPPH radical scavenger and to inhibit lipid peroxidation. ${ }^{246,247}$ In fact, phloretin shows greater antioxidant capacity than flavanones with corresponding structures and functional groups. ${ }^{247}$

1.4.4.3 Anticancer Activity Numerous groups have examined the in vitro and in vivo anticancer activity of phloretin and/or phloridzin. This established activity may be attributed to two possible mechanisms. First, the ability of phloretin and phloridzin to inhibit glucose transport, and second, the ability of phloretin to inhibit P-glycoprotein. Additionally, phloretin may alter the expression of apoptosis regulatory proteins. It should be noted that phloretin has been shown to be nontoxic to HUVECs. ${ }^{262}$ Reports of phloretin's and phloridzin's anticancer activities are summarized below.

Phloretin and phloridzin have shown in vivo activity in rat mammary adenocarcinoma and Fischer bladder cell carcinoma cell lines as determined by a decrease in tumor diameters in comparison to controls. ${ }^{248}$ This result supports the ability of phloretin and phloridzin to block glucose transport into tumor cells in vitro and tumor tissues in vivo. ${ }^{249}$ Kobori et al. found phloretin induced apoptosis in B16 mice melanoma 4A5 cells. These results were contributed to the inhibition of glucose transmembrane transport by phloretin and possible promotion of Bax (Bcl-associated $\mathrm{X}$ ) protein expression and caspase activation. ${ }^{250}$ The same research group showed phloretin induces apoptosis in HL60 human leukemia cells that was attributed to inhibition of protein kinase $\mathrm{C}$ activity. ${ }^{263}$ Park et al. reported apoptosis in HT29 cells by phloretin through activation of caspases and promotion of Bax expression. ${ }^{264} \mathrm{Kim}$ et al. found phloretin induces apoptosis in H-Ras-transformed MCF10A human breast epithelial (H-Ras MCF10A) cells though inducing JNK, p38, and caspase-3. ${ }^{265}$ Additionally, phloretin has been show to potentiate the anticancer actions of paclitaxel in both in vitro grown HEP-G2 cells and in vivo in xenografted mice. Phloretin was also shown to resensitize liver cancer cells to the effects of paclitaxel and to induce apoptosis. ${ }^{253}$

Phloretin is suggested to be a P-glycoprotein inhibitor. Zhang et al. demonstrated an increase in accumulation of daunomycin in P-glycoprotein-overexpressing MCF-7/ADR cells incubated with phloretin. ${ }^{266}$ Phloretin has also been shown to inhibit multi-drug resistant (MDR) efflux pumps in human MDR1 gene-transfected mouse lymphoma cells and human breast cancer cells (MDA-MB-231) and Panc-1 cells expressing the MRP1 pump. ${ }^{251,252}$ Phloretin's P-glycoprotein inhibition activity was further confirmed in MCF-7 and MDA435/LCC6 cell lines. ${ }^{267}$ 
1.4.4.4 Antiinflammatory Activity Phloretin inhibited proinflammatory gene expression in studies by Jung et al. This study demonstrated phloretin represses NF- $\kappa \mathrm{B}$, IP-10-, IL-8-promoter-, and STAT1-dependent signal transduction in a dose-dependent manner. ${ }^{268}$ Phloretin has also been shown to inhibit mouse T-lymphocyte proliferation. ${ }^{269}$

1.4.4.5 Other Activities Other pharmacological properties of phloretin are starting to be elucidated. Phloretin and phloridzin have been shown to act as phytoestrogens. ${ }^{254}$ Additionally, in a study by Stangl et al., phloretin was shown to possess antithrombotic properties. Phloretin suppressed the stimulation of three endothelial adhesion molecules including ICAM-1, VCAM-1, and endothelial leukocyte adhesion molecule-1 (E-selectin) ${ }^{262}$ Other studies have shown that apple juices were able to inhibit cytochrome P450 1A1 induction, which may confer reduced activation of certain chemical carcinogens. ${ }^{270}$

\subsubsection{Homoeriodictyol}

Homoeriodictyol (+/-3'-O-methyl-eriodictyol;+/-5,7,4'-trihydroxy-3'methoxyflavanone; $\mathrm{C}_{16} \mathrm{H}_{14} \mathrm{O}_{6} ; \mathrm{MW}=302.27 \mathrm{~g} / \mathrm{mol} ; \mathrm{XLog} \mathrm{P}=1.1$ ) is a chiral flavanone consumed in citrus fruits and herbal products. ${ }^{93}$ Homoeridictyol and its glycosides have been successfully identified or extracted from several plants in a variety of botanical families including Anacardiaceae $\left(R h u s^{271}\right)$, Asteraceae (Lychnophora ${ }^{272}$ ), Hydrophyllaceae (Eriodictyon ${ }^{62,273}$ ), Loranthaceae (Viscum $\left.{ }^{274-277}\right)$, Poaceae $\left(\mathrm{Zea}^{278}\right)$, and Rutaceae $\left(\right.$ Citrus $\left.^{52}\right)$.

Homoeriodictyol and its analogs have been commercially used as flavor modifiers. ${ }^{273}$ Products made from yerba santa have been used in the pharmaceutical industry as bitter remedies for several years. However, these products may not be suitable for many food or pharmaceutical applications because they are too aromatic. Homoeriodictyol, a constituent of yerba santa, and its sodium salt have been used in sensory studies and have been shown to significantly decrease the bitter taste of caffeine without interfering with the desired intrinsic flavors or taste characteristics. ${ }^{273}$ Moreover, homoeriodictyol sodium salt has been further investigated for its bitter masking properties in different chemical classes of bitter molecules. ${ }^{273}$

Yerba santa (Eriodictyon glutinosum) is also commercially available. ${ }^{60}$ It has been used for the treatment of the common cold and asthma. ${ }^{273}$ In the late nineteenth century, alcoholic extracts of yerba santa were used as masking agents for quinine. Currently, yerba santa is being used to enhance the moisturizing and lubricating properties of cosmetic, medical, and dental products. $^{60}$

Biosynthesis of homoeriodictyol has been previously studied. ${ }^{278-280} \mathrm{Mc}-$ Cormick first described homoeridictyol as a precursor of the anthocyanin peonidin, a pigment found in both immature and mature seeds in mutant maize aleurone tissue. ${ }^{278}$ Subsequently, in 2003, Ibrahim et al. described the fungus Cunninghamella elegans as capable of converting 5,4'-dihydroxy-7,3'- 
dimethoxyflavanone into both homoeriodictyol and homoeriodictyol-7sulfate. ${ }^{279}$ The importance of this study was its contribution to the understanding of the possible similarities between mammalian and microbial systems in phase II conjugation reactions as a novel tool in metabolic drug investigations. C. elegans carried out C-7 demethylation, and sulfation of 5,4'-dihydroxy-7,3'dimethoxyflavanone to successfully produce the flavanone homoeriodictyol and its sulfoconjugate. ${ }^{279}$ In addition, methylation reactions have also been described to be a part of the biosynthesis of flavonoids. ${ }^{280}$ For example, the flavonoids detected in Catharanthus roseus have a simple methylation pattern; methyl groups in positions $3^{\prime}$ and $5^{\prime}$ are introduced by an unusual $O$ methyltransferase that performs two consecutive methylations in the B-ring. A recently identified $O$-methyltransferase (CrOMT6) was described to methylate the B-ring at $4^{\prime}$ position and, in collaboration with dioxygenases, facilitates the conversion of flavanones into flavones, dihydroflavonols, and flavonols. ${ }^{280}$ Homoeriodictyol was reported to be the preferred substrate for CrOMT6, and depending on the acting dioxygenases, the corresponding flavone (flavone synthase), dihydroflavonol (flavanone $3 \beta$-hydroxylase), or flavonol (flavonol synthase [FLS], anthocyanidin synthase) resulted as a product.

1.4.5.1 Pharmacokinetic Studies Homoeridictyol and its glucuro- and sulfoconjugates have been detected as metabolites in plasma and/or urine after the oral administration of flavanone,${ }^{54}$ hesperidin ${ }^{281}$ or eriocitrin ${ }^{55}$ in rats and in humans. Flavanone glycosides or aglycones were administered to healthy male humans, and plasma was analyzed for metabolites using high performance liquid chromatography (HPLC); homoeriodictyol was detected only in samples of volunteers receiving flavanone glycosides but not in those who received flavanone aglycones. ${ }^{54}$ Similarly, hesperidin was orally administered to rats, and plasma was analyzed using liquid chromatography-mass spectrometry (LC-MS); homoeriodictyol was detected as a monoglucuronide and as a sulfate metabolite. ${ }^{281}$ In another study, eriocitrin was orally administered to rats, and plasma and urine were analyzed using HPLC and LC-MS; both homoeriodictyol and its glucuroconjugate were detected. ${ }^{55}$

To our knowledge, only one study has examined the pharmacokinetics of homoeriodictyol in rats. Booth et al. administered racemic homoeriodictyol at a dose of $150 \mathrm{mg} / \mathrm{rat}$ and used paper chromatography for the analysis of homoeriodictyol metabolites in urine. ${ }^{282}$ Homoeriodictyol and its glucuroconjugates, m-hydroxyphenylpropionic acid, m-coumaric acid, and dihydrofurelic acid, were detected in urine after oral administration of homoeriodictyol. However, no stereospecific analysis or pharmacokinetic disposition parameters was reported. In another study, homoeriodictyol-7- $O-\beta$-D-glucopyranoside (HEDT-Glu) was administered to male and female rats via intravenous (IV) injection, and urine and tissues were analyzed via HPLC ${ }^{276}$ or LC-MS. ${ }^{283}$ The previously developed analytical assays also detected homoeridictyol, but neither reported enantioseparation of homoeriodictyol enantiomers. 
Pharmacokinetic parameters and tissue distribution values were reported for HEDT-Glu and homoeriodictyol, but individual enantiomers were not analyzed. Plasma concentrations of HEDT-Glu in rat were detectable for at least 5 hours after IV administration; HEDT-Glu was cleared from the blood and was distributed mainly to the liver and small intestine; at 0.083 hours postdose, the concentrations of HEDT-Glu in these tissues were $0.65 \pm 0.24 \mu \mathrm{g} / \mathrm{g}$ and $0.51 \pm 0.07 \mu \mathrm{g} / \mathrm{g}$, respectively. ${ }^{276}$ In comparison, homoeriodictyol was mainly detected in the kidney, reaching $10.93 \pm 2.92 \mu \mathrm{g} / \mathrm{g}$ at 0.083 hours postdose. ${ }^{283}$

1.4.5.2 Pharmacological Activity Homoeriodictyol and its glycosides have been described to possess antimicrobial, ${ }^{271}$ antioxidant,${ }^{55}$ anticancer, ${ }^{283-285}$ antiinflammatory, ${ }^{276}$ antifungal, ${ }^{283}$ and antiosteoporotic ${ }^{275}$ activity. Homoeriodictyol was also described to increase coronary flow rate, ${ }^{283}$ decrease platelet aggregation, ${ }^{283}$ and act as a bitter masking or sweet enhancing agent. ${ }^{286}$

\subsubsection{Isosakuranetin}

Isosakuranetin (+/- 4'-methylnaringenin; +/- 4'-methoxy-5,7-dihydroxyflavanone; +/- ponciretin; $\mathrm{C}_{16} \mathrm{H}_{14} \mathrm{O}_{5} ; \mathrm{MW}=286.28 \mathrm{~g} / \mathrm{mol}$; $\mathrm{XLogP}=2.3$ ) is a flavanone flavonoid with two enantiomeric forms: $2 \mathrm{~S}$ - and $2 \mathrm{R}$-isosakuranetin (Fig. 1.16). This flavanone has been identified as an important component of propolis, ${ }^{287-289}$ and several plants found in divergent botanical families including Asteraceae (Baccharis ${ }^{290,291}$ ), Combretaceae (Terminalia ${ }^{292}$ ), Eupatorieae (Chromolaena ${ }^{293}$ and Eupatorium ${ }^{294}$ ), and Rutaceae (Citrus ${ }^{295}$ ). Didymin (2S- isosakuranetin-7-rutinoside) and poncirin (2S-isosakuranetin7-neohesperidoside), two main glycosides of isosakuranetin (Fig. 1.17), have been reported exclusively in Rutaceae (Citrus ${ }^{295-299}$ and Poncirus ${ }^{300-305}$ ).

Flavanones in nature are found mostly as glycosides, attached to $\beta$ neohesperidose or $\beta$-rutinose sugars through the C-7 hydroxyl group. ${ }^{298,306}$ The flavanone neohesperidosides and rutinosides can be easily distinguished by their taste properties: The neohesperidosides are bitter, whereas the rutinosides are tasteless. ${ }^{306}$ Hot alkali on $7-\beta$-neohesperidosides splits off the B-ring and carbon-2 to yield phloracetophenone 4 '- $\beta$-neohesperidoside; however, 7 - $\beta$-rutinosides do not display phloracetophenone 4 '- $\beta$-rutinoside formation when exposed to hot alkali but instead generate a sugar-aglycone bond split. ${ }^{306}$<smiles>COc1ccc([C@H]2CC(=O)c3c(O)cc(O)cc3O2)cc1</smiles>

Figure 1.16. Chemical structure of isosakuranetin enantiomers, $2 \mathrm{~S}$-isosakuranetin (left) and 2R-isosakuranetin (right). 
<smiles>COc1ccc([C@H]2CC(=O)c3c(O)cc(O[C@@H]4O[C@H](CO)[C@@H](O)[C@H](O)[C@H]4O[C@H]4O[C@H](C)[C@@H](O)[C@H](O)[C@H]4O)cc3O2)cc1</smiles><smiles>COc1ccc([C@H]2CC(=O)c3c(O)cc(O[C@@H]4O[C@H](CO[C@@H]5O[C@@H](C)[C@H](O)[C@H](O)[C@H]5O)[C@@H](O)[C@H](O)[C@H]4O)cc3O2)cc1</smiles>

Figure 1.17. Isosakuranetin glycosides (1) poncirin (2S-isosakuranetin-7-neohesperidoside) and (2) didymin (2S-isosakuranetin-7-rutinoside).

Isosakuranetin has been included in its glycosylated form (isosakuranetin7 - $\beta$-rutinoside) in dietary supplements, vitamins, skin care products, energy drinks and so forth. ${ }^{60}$ Isosakuranetin is a major component of propolis. Propolis is a natural resinous substance made by honeybees from plant exudates and used to protect honeycombs against intruders. ${ }^{290}$ The composition of propolis depends on the plants in the region and the season in which it is collected by the bees. Propolis has been used in folk medicine and is currently studied for its biological activities. Currently, propolis is extensively incorporated in food and beverages as a dietary supplement. ${ }^{290}$

Biosynthesis of isosakuranetin has been poorly studied. ${ }^{307,308}$ Preliminary evidence of the existence of a "flavanone synthase," which converts chalcone glycosides into flavanone glycosides, was presented in 1956. The enzymatic activity of flavanone synthase responsible for poncirin chalcone's conversion into poncirin was studied using various sources including Citrus, Poncirus, Cosmos, and Dahlia. Peel tissue from Citrus aurantium showed the highest flavanone synthase activity. ${ }^{308}$ Subsequently, Kim et al. demonstrated the existence of SOMT-2, a soybean (Glycine max, Fabaceae) $O$-methyltransferase expressed in $E$. coli capable of converting naringenin into isosakuranetin by methylation at the $4^{\prime}$-hydroxyl position. ${ }^{307} O$-Methylation of flavonoids has been described to alter the chemical reactivity of their phenolic hydroxyl groups and to enhance their lipophilicity. 
1.4.6.1 Pharmacokinetic Studies Isosakuranetin and its glucuroconjugates have been previously detected as metabolites in rats administered the flavonoid naringin. ${ }^{309}$ According to Silberberg et al., the methylation of 4'-hydroxyl in naringin produced isosakuranetin since aromatic flavonoid compounds can undergo methylation, hydroxylation, and demethylation reactions via bacterial metabolism in the large intestine. Both healthy rats and rats bearing Yoshida's sarcoma cells produce isosakuranetin and its glucuronides in plasma, urine, liver, and kidney; however, lower concentrations were detected in tumorbearing rats. A reduction in tumor concentration of flavonoids could be the result of multi-drug resistance-associated protein (MRP) activity, for which flavonoids may act as substrates. ${ }^{309}$

Metabolism of flavonoids has been described to occur in intestinal microflora. Poncirin, for example, is converted to isosakuranetin, ${ }^{310} 4$-hydroxybenzoic acid; 2,4-dihydroxyacetophenone; phloroglucinol; and pyrogallol by human intestinal microflora in vitro, in particular: Fusobacterium K-60, Eubacterium YK-4, and Bacteroides JY-6. ${ }^{311}$ Isosakuranetin was further converted to phenolic acid by Streptococcus S-1, Lactobacillus L-2, Bifidobacterium B-9, and Bacteroides JY-6. ${ }^{311}$ Shimuzu et al. demonstrated that isosakuranetin in propolis extracts can be incorporated into intestinal Caco-2 cells and transported from the apical to the basolateral side in vitro. ${ }^{287}$ These findings are valuable for studies related to intestinal cell function involved in absorption from the gastrointestinal tract. To our knowledge, there are no pharmacokinetic studies of isosakuranetin that acknowledge the importance of its chiral nature and disposition.

1.4.6.2 Pharmacological Activity Isosakuranetin has been previously described to have antimycobacterial, ${ }^{293}$ antifungal, ${ }^{312}$ antioxidant, ${ }^{288,313}$ antibacterial, ${ }^{303}$ neuroprotective, ${ }^{314}$ enteroprotective, ${ }^{108,303}$ anticancer, ${ }^{292,303,311}$ and antiallergic ${ }^{305}$ properties. Poncirin and didymin were found to have numerous biological activities such as antiinflammatory, ${ }^{300,301,304}$ antioxidant, ${ }^{313}$ anticancer, ${ }^{311}$ antiplatelet,${ }^{311}$ antiatherogenic, ${ }^{315}$ and immunomodulatory ${ }^{316}$ properties. However, there is a lack of information regarding the stereospecific activity or disposition of isosakuranetin enantiomers in biological matrices like urine and serum. Achiral analysis of isosakuranetin may be misleading in that absorption, distribution, metabolism, and elimination may all be stereoselective processes. Measuring enantiomers may facilitate the establishment of more meaningful concentration effect relationships of chiral drugs. Separation of enantiomers in biological matrices is thus important to comprehensively understand the stereospecificity of action and disposition of isosakuranetin.

\subsubsection{Taxifolin}

Racemic taxifolin (+/- 3,5,7,3,3, $4^{\prime}$-pentahydroxyflavanone; +/- dihydroquercetin; $\mathrm{C}_{15} \mathrm{H}_{12} \mathrm{O}_{7} ; \mathrm{MW}=304.25 \mathrm{~g} / \mathrm{mol} ; \mathrm{XLogP}=0.79-3.73^{317}$ ), a dihydroflavonol, and its glycosides have been previously identified in plants included 
in a variety of botanical families including Alliaceae (Allium ${ }^{318}$ ); Annonaceae (Cleistopholis ${ }^{319}$ ); Apocynaceae (Trachelospermum ${ }^{320}$ ); Asteraceae (Silybum, ${ }^{321,322}$ Tessaria ${ }^{323}$ Centaurea,${ }^{324}$ and Proustia $\left.{ }^{325}\right) ;$ Cactaceae $\left(\right.$ Opuntia $\left.^{326}\right)$; Clusiaceae (Garcinia ${ }^{327}$ and Hypericum ${ }^{328}$ ); Cupressaceae (Chamaecyparis ${ }^{329}$ and Thujopsis ${ }^{330}$ ); Ericaceae (Rhododendron ${ }^{331}$ ); Fabaceae (Acacia, ${ }^{332}$ Genista $^{333}$ and Trifolium ${ }^{334}$ ); Juglandaceae (Englehardtia ${ }^{335}$ ); Lamiaceae (Origanum $^{147}$ and Thymus $\left.{ }^{212}\right)$; Liliaceae $\left(\right.$ Rhyzoma $\left.^{336-340}\right)$; Loranthaceae $\left(\right.$ Taxillus $\left.^{341}\right)$; Ochnaceae $\left(\right.$ Ochna $\left.^{342}\right)$; Oleaceae $\left(\right.$ Olea $\left.^{343}\right)$; Pinaceae (Picea ${ }^{344}$ Pinus ${ }^{345-347}$ Larix ${ }^{348,349}$ and Pseudotsuga ${ }^{349}$ ); Poaceae $\left(\right.$ Fussia $^{350}$ ); Polygonaceae (Polygonum $\left.^{351}\right)$; Proteaceae (Helicia $\left.{ }^{352}\right)$; Smilacaceae (Smilax ${ }^{340,353,354}$ ); Solanaceae $\left(\right.$ Petunia $^{355}$ ); and Vitaceae (Ampelopsis ${ }^{356}$ and Vitis $^{357}$ ). Likewise, several cultivars of wine have been analyzed for their taxifolin content. ${ }^{358,359}$ The most widely studied of these plants is Rhizoma smilacis glabrae or tu fu ling, which has been used in traditional Chinese medicine to treat cancer and acquired immune deficiency syndrome (AIDS) patients. ${ }^{337}$ Clinically, taxifolin has been used to treat several illnesses including infection of the urinary system, leptopirosis, dermatitis, brucellosis, eczema, acute bacterial dysentery, acute and chronic nephritis, syphilis, arthritis, and folliculitis. ${ }^{336-340}$ Taxifolin has been successfully isolated from $R$. smilacis glabrae showing high extraction efficiency by sonication and use of hot solvents. Chen et al. did not accomplish total enantiomeric separation of the four taxifolin enantiomers using an HPLC method. ${ }^{337}$ Nevertheless, the separation and identification of the four glycosylated taxifolin enantiomers (neoastilbin, astilbin, neoisoastilbin, and isoastilbin; Fig. 1.18) and racemic taxifolin was attained in this study. ${ }^{337}$

Taxifolin has been reported to be a potent antioxidant and has been used as a biological active supplement in the food industry. ${ }^{360}$ Taxifolin is commercially available as a food additive and is used in vegetable oils, milk powder, pastry, and so forth. Plants like French maritime pine bark (Pinus pinaster) and katsura (Cercidiphyllum japonicum), in which taxifolin is a major component, are currently being investigated. An extract of French maritime pine bark (P. pinaster), Pycnogenol ${ }^{\circledR}$, is being used in the treatment of attention deficit<smiles></smiles>

Figure 1.18. Chemical structures of a taxifolin rhamnoside, astilbin. 
hyperactivity disorder (ADHD) in Europe with positive results. ${ }^{347}$ Pycnogenol has been demonstrated to stimulate endothelial nitric oxide synthase. Increased production of nitric oxide (NO) may improve brain functions such as memory, learning, and modulation of wakefulness. ${ }^{347}$ Likewise, katsura (C. japonicum) has been reported as an effective hair growth control agent. ${ }^{361}$ Polyphenolic compounds in katsura showed proliferation of mouse epithelial cells in vitro that are currently being investigated as accelerators of hair regrowth. ${ }^{347}$

Biosynthesis of taxifolin has been previously studied. Brignolas et al. reported the synthesis of taxifolin glycoside in a fungus-resistant clone of Norway spruce (Picea abies Karst.) after inoculation with Ophiostoma polinicum Siem, a pathogenic fungus associated with a bark beetle, Ips typographus L., but not after sterile inoculation or in an unwounded clone. These findings suggest the flavonoid pathway may be involved in resistance to pathogenic fungi. In that report, CHS activity was described to be higher in the resistant clone than in a clone susceptible to the pathogenic fungus. ${ }^{344}$ Enzymes like CHS have been described to play a role in the biosynthesis of flavonoids. For example, production of rutin was described following administration of exogenous taxifolin (Fig. 1.19) in Satsuma mandarin (Citrus unshiu) peel tissues, which demonstrated the ability of peel tissue to convert dihydroflavonols into flavonol glycosides. FLS increased in peel during maturation, unlike other enzymes involved in flavonoid biosynthesis including CHS, CHI, and flavanone 3-hydroxylase $(\mathrm{F} 3 \mathrm{H}){ }^{362}$

Taxifolin has been described as an intermediate in the biosynthesis of other flavonoids. In this matter, it has been reported that of the four phenolic hydroxyl groups, $7-\mathrm{OH}$ is the most acidic and 5-OH the least acidic. ${ }^{323}$ These findings are important for the determination of the methylation pattern and the possible metabolic products of taxifolin methylation. In Centaurea maculosa roots, for example, kaempferol was converted into taxifolin, which, in turn, was converted into catechin. These three flavonoids were described as phytotoxic root exudates produced by C. maculosa. ${ }^{324}$ In comparison, Matsuda et al. described the biotransformation of catechin into taxifolin by Burkholderia sp KTC-1. This biotransformation occurred in two steps: 4-hydroxylation, and dehydrogenation with the formation of leucocyanidin as an intermediate. $^{363}( \pm)$-Catechin 4-hydroxylase and leucocyanidin 4-dehydrogenase were described to accumulate in the cytosol of the aerobic bacteria used in this study.

Only one study considers the isomerization of taxifolin. ${ }^{364}$ (2R3R)-Taxifolin was converted into (2S3R)-taxifolin with the opening of the heterocyclic ring and the formation of an intermediate quinine methide with heat $<100^{\circ} \mathrm{C}$. When acidic or basic methylation was used under heat $>100^{\circ} \mathrm{C}$, isomerization did not occur, and alphitonin was formed. Alphitonin (2-benzyl-2,3',4,4',6pentahydroxy-3-coumaranone) is a by-product of taxifolin methylation. ${ }^{364}$

1.4.7.1 Pharmacokinetic Studies There are a paucity of studies on the pharmacokinetics of taxifolin. ${ }^{365,366}$ A pharmacokinetic analysis of maritime 


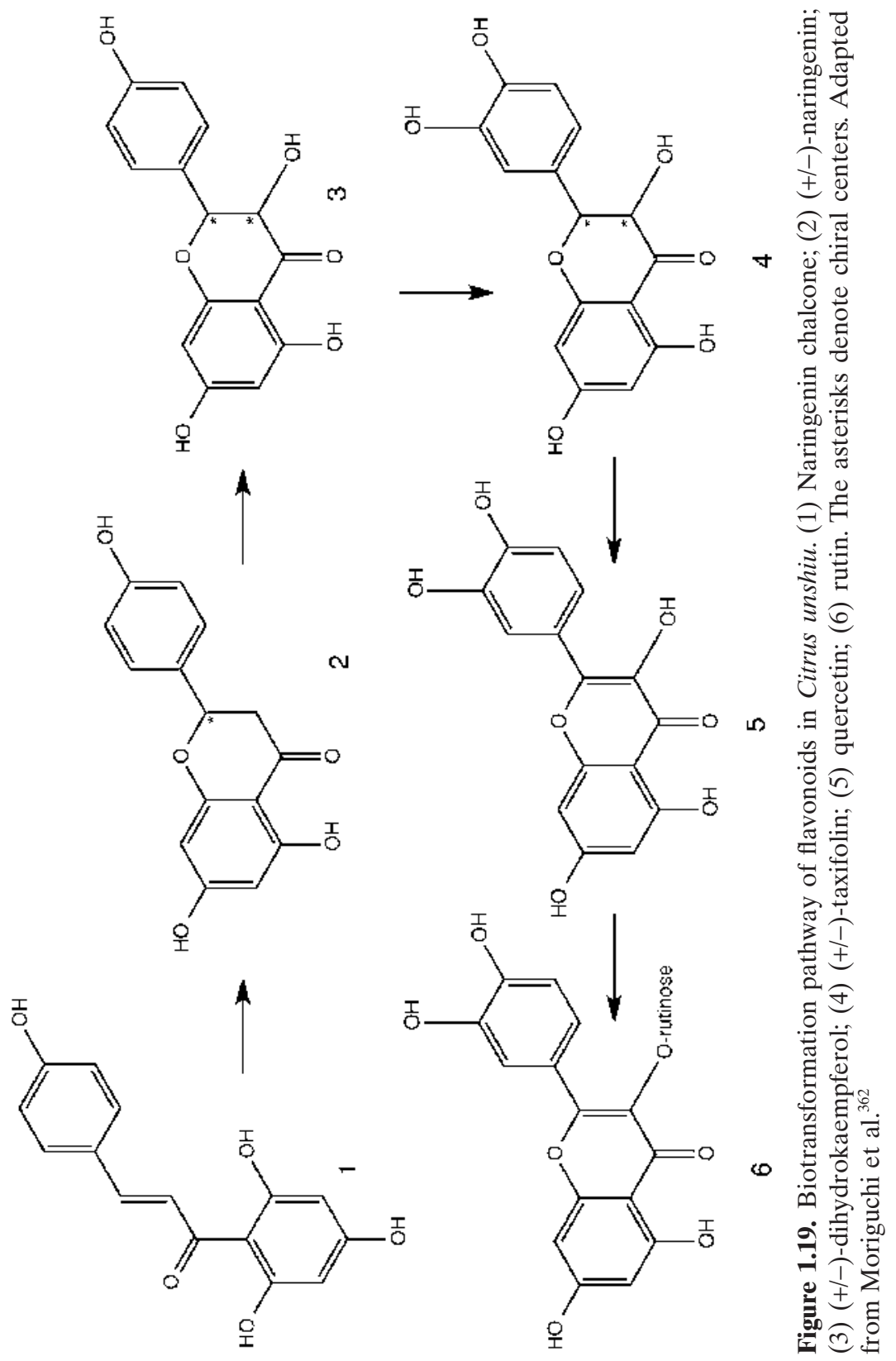


pine bark extract demonstrated the presence of taxifolin in human plasma after single and multiple doses of the extract were administered orally $\left(\mathrm{AUC}_{[0-t]}\right.$ $=2311.11 \pm 85.98 \mathrm{ng} / \mathrm{mL} \times \mathrm{h} ; \quad C_{\max }=33.34 \pm 12.54 \mathrm{ng} / \mathrm{mL} ; \quad t_{\max }=8.2 \pm 2.5$ hours). ${ }^{366}$ Likewise, after oral administration of Pycnogenol, the active constituent of maritime pine bark, taxifolin was detected in human urine. ${ }^{365}$ Flavonoid metabolism in humans is known to involve the intestinal microflora, ${ }^{367,368}$ as well as liver enzymes. ${ }^{369}$ Among the microflora shown to participate in the metabolism of taxifolin, Eubacterium ramulus and Clostridium orbiscindens have been described to convert taxifolin into phenolic acids. E. ramulus is found in human feces and has been described to convert quercetin into taxifolin, which, in turn, is converted into its chalcone; following a series of additional conversions, taxifolin is finally converted into 3,4-dihydroxyphenylacetic acid $^{367}$ (Fig. 1.20). Likewise, C. orbiscindens is found in human feces and has also been described to have the ability to convert taxifolin into 3,4dihydroxyphenylacetic acid ${ }^{368}$ (Fig. 1.21). However, C. orbiscindens is an asaccharolytic organism that relies on the deglycosilation performed by human tissues (small intestine, liver) and bacteria such as E. ramulus, Enterococcus casseliflavus, and Bacteroides sp. for flavonoid degradation. ${ }^{368}$ Flavonoid metabolism by liver enzymes has been studied by Nielsen et al.; in their study, cytochrome P450 activity did not appear to be involved in taxifolin metabolism in rat liver microsomes. ${ }^{369}$ Nielsen et al. described the structural characteristics and the tentative products of flavonoids metabolized by liver enzymes: (1) Flavonoids without a $4^{\prime}$-hydroxyl group in the B ring undergo hydroxylation by microsomal enzymes to catechol (3',4'-dihydroxyl) structures; (2) flavonoids with a $4^{\prime}$-methoxyl group, but not those with a methoxyl in the $3^{\prime}$-position, undergo demethylation into the hydroxyl compound (i.e., isosakuranetin); and (3) flavonoids with two or more hydroxyl groups in the B ring or a 3'-methoxyl group are not metabolized by microsomal enzymes (i.e., homoeriodictyol, taxifolin). In addition, cytochrome P450s involved in flavonoid metabolism have been described to exhibit stereoselectivity. ${ }^{369}$

1.4.7.2 Pharmacological Activity Several of the plants described to possess taxifolin are used in traditional and clinical medicine, $212,319,322,325-327,335-338$, $346,349,351,352,370$ some are ingested in the human diet; ${ }^{212,318,334,351,357-359,362,371}$ and others are being studied for their potential use in drug development. ${ }^{333,342}$

Racemic taxifolin and its glycosides have been previously studied for their potent antioxidant properties. $325,335,346,349,370,372$ Taxifolin is a very common antioxidant additive in the food industry ${ }^{212}$ and has also been described to have antiinflammatory and analgesic properties, ${ }^{325}$ hepatoprotective capacity, ${ }^{373}$ free radical scavenger activity, ${ }^{21,335,349,374}$ and also demonstrates a protective role in plants against pathogens. ${ }^{32,344}(2 \mathrm{R} 3 \mathrm{R})-(2 \mathrm{R} 3 \mathrm{R})-(+)$-Taxifolin, one of its four enantiomers, has been described to possess tyrosinase inhibitory capacity, and thus it is used in depigmentation drugs and whitening cosmetics, as well as a food additive and an insect control agent. ${ }^{351}$ 


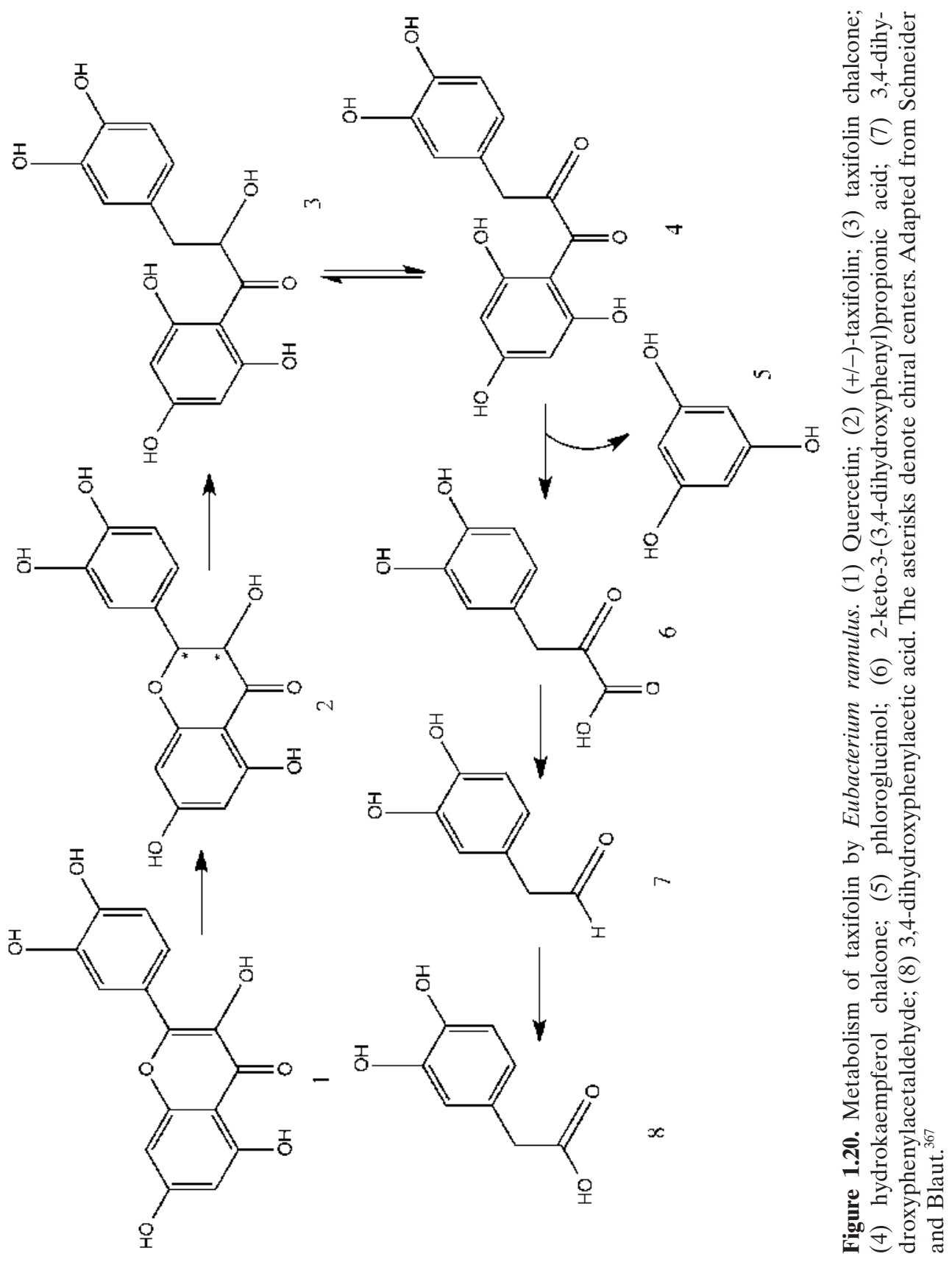




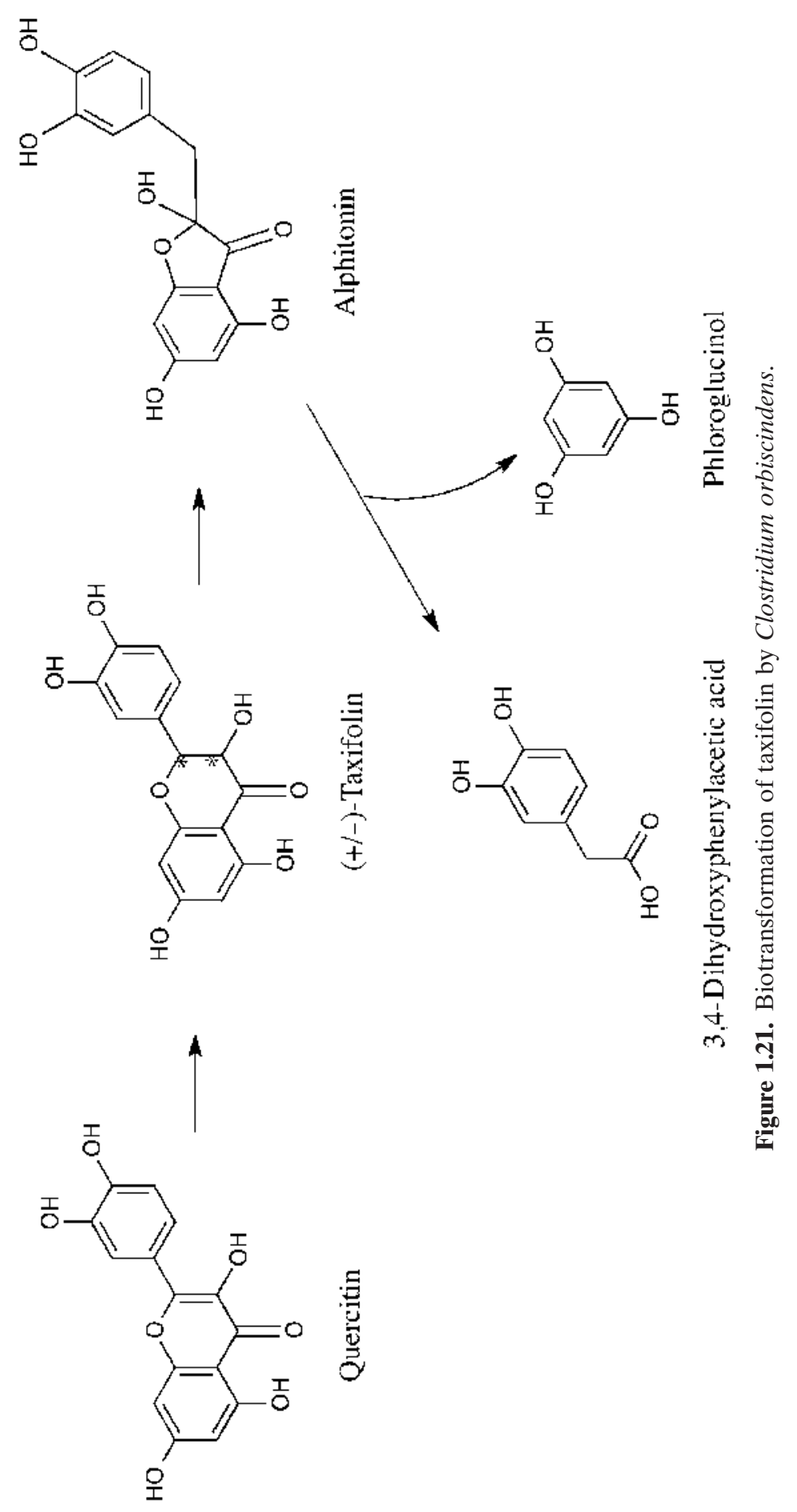




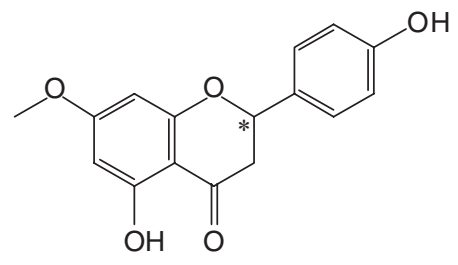

Figure 1.22. Structure of sakuranetin. The asterisk indicates a chiral center.

\subsubsection{Sakuranetin}

Sakuranetin (Fig. 1.22), [(+/-)7-O-methylnaringenin $],{ }^{279}$ 2,3-dihydro-5hydroxy-2-(4-hydroxyphenyl)-7-methoxy-4H-1-benzopyran-4-one), ${ }^{375}$ 5-hydroxy-2-(4-hydroxyphenyl)-7-methoxy-2,3-dihydro-4H-chromen-4one, ${ }^{376}$ or $\left[(+/-) 5,4^{\prime} \text {-dihydroxy-7-O-methoxyflavanone }\right]^{377,378}$ is a chiral flavanone ${ }^{285,379-381}$ phytoalexin $^{382,383}$ that has been detected or isolated from numerous plants including rice (Oryza sativa L.), ${ }^{377,381,384-386}$ fingerroot (Boesenbergia pandurata) ${ }^{378}$ Dodonaea viscose (L.) Jacq. (Sapindaceae) ${ }^{387}$ yerba santa (Eriodictyon californicum), ${ }^{285}$ Eucalyptus maculate Hook, ${ }^{388}$ Larrea tridentate, ${ }^{389}$ Loranthaceae (Phoradendron robinsonii), ${ }^{376}$ spiked pepper (Piper aduncum), ${ }^{379}$ Piper crassinervium, ${ }^{380}$ Piper lhotzkyanum, ${ }^{390}$ Ongokea gore,${ }^{391}$ P. davidiana, ${ }^{228}$ propolis, ${ }^{392}$ Pruni Cortex (Prunus jamasakura Siebold) ${ }^{393}$ and Xanthorrhoea arborea. ${ }^{394}$ Sakuranetin was named after being first isolated as the aglycone of sakuranin from the bark of the cherry tree, which is affectionately associated with cherry blossoms (sakura). ${ }^{375,395}$ All of these plants have been associated with medicinal use (treatment of cancer, pain, inflammation, asthma, diabetes, etc.) in places such as Japan, China, India, and Mexico. Stereospecific quantification has not been performed in previous investigations of this chiral flavanone.

Induction of sakuranetin was achieved by UV light, ${ }^{377,383,384,396-398}$ blast infection, ${ }^{377,384}$ copper chloride,,$^{377,384,396,399}$ amino acid conjugates of jasmonic acid, ${ }^{377,385,396}$ methionine, ${ }^{396,399}$ coronatine, ${ }^{400}$ and chitosan oligomers ${ }^{396}$ in rice leaves. This induction of sakuranetin could be counteracted by tiron, ${ }^{399}$ kinetin, ${ }^{400}$ and zeatin. ${ }^{400}$ Production of sakuranetin in rice cells ${ }^{384,400}$ and rice leaves ${ }^{384,399,400}$ was accomplished by endogenously applying jasmonic acid, ethylene, and ethephon. Sakuranetin has been synthesized, induced, and produced in a variety of ways. Synthesis of sakuranetin derived from naringenin involves naringenin 7-O-methyltransferase in plants. ${ }^{377,384,385}$

\subsubsection{Gallic Acid}

There has been an increased interest in both the study and consumption of natural products as evidenced by the increase in nutraceutical sales and the practice of alternative medicine worldwide. ${ }^{2,4,5,8401}$ Use of these products is 
substantial through health and beauty, dietary supplement, performance enhancement supplements, food and beverage, to overall health and wellbeing products. These xenobiotics can be extracted from plants or can act as precursors to drugs that can be further modified, synthesized, formulated, manufactured, and subsequently sold for their reported health benefits. Through evidence-based pharmaceutical and medical research, a better understanding of how or whether natural products can be used as therapeutics can be attained.

Secondary plant metabolites such as tannins are well known and typically have important roles in plant-plant and plant-animal interaction roles, more specifically in adaptation and esthetics. ${ }^{402}$ Tannins are appreciated by beer and wine connoisseurs due to their abundance in these beverages. The popularity of wine, particularly in France, has led to extensive studies of wine content. The phenomena widely known as the "French paradox" is the supposition that a regular intake of red wine in the diet may, because of its constituent phytochemicals and their protective effects, allow for the consumption of saturated fats without a high mortality from coronary heart disease (CHD). ${ }^{23,27}$ The exact mechanism of the French paradox has not been established; however, research thus far has indicated correlations with reactive oxygen and reactive nitrogen species scavenging consistent with polyphenol consumption.

Hydrolyzable tannins, as opposed to condensed tannins, are a subsection of plant tannins that can be described as esters of gallic acid. ${ }^{403} \beta$-Glucogallin is also referred to as [(2S,3R,4S,5S,6R)-3,4,5-trihydroxy-6-(hydroxymethyl) oxan-2-yl] 3,4,5-trihydroxybenzoate, 1 -galloylglucose, 1 -galloyl- $\beta$-glucose, 1 -galloyl-glucose, and 1-O-galloyl- $\beta$-D-glucose, while gallic acid is identified as gallate and 3,4,5 trihydroxybenzoic acid (Fig. 1.23). $\beta$-Glucogallin and/or gallic acid have been detected and/or isolated from a variety of botanicals; however, the coelution of deleterious matter are often present in these studies. ${ }^{404-409}$ Amla, also known as the Indian gooseberry, is widely consumed as a fruit in India and is highly marketed as the major constituent of numerous health and beauty products. As $\beta$-glucogallin is thought to be a major component of amla

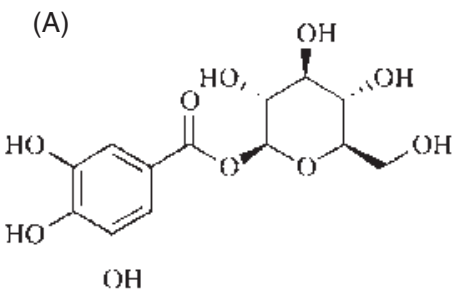

(B)<smiles>O=C(O)C1=CC(O)C(O)C(O)=C1</smiles>

Figure 1.23. Structure of (A) $\beta$-glucogallin and (B) gallic acid. 
and as the popularity of nutraceuticals has increased, there is a need to characterize the pharmacokinetics of these compounds.

Sources of $\beta$-glucogallin and gallic acid have been utilized in traditional folk medicines as skin lighteners and other beauty esthetics, in the treatment for skin disorders, and for termination of hemorrhages. Gallic acid is readily used in the pharmaceutical industry as an indicator of total phenol content and is a starting molecule for the synthesis of gallates.

\subsection{CONCLUSIONS}

Flavonoids are a large family of compounds that, as presented, follow a complex biosynthesis in plants that depend on host, the region of origin, and the environmental stimuli. Furthermore, it has been presented that these compounds have been studied for over 80 years, and multiple pharmacological activities have been identified in vitro and preclinically. These activities make them very attractive to pharmaceutical and nutraceutical companies that can use them by modifying their structures to improve efficacy and specificity. However, many of the studies have overlooked the fact that many of these flavonoids are chiral, which warrants the need for more studies that could allow these compounds to eventually move into the clinic and be able to obtain plant-derived drugs in the market.

\section{REFERENCES}

1. Bailey, R. (2003). Nutraceuticals sales to hit $\$ 75$ billion, Decision News Media SAS.

2. Bailey, R. (2008). Japan: An established market for nutraceuticals. Nutraceuticals World. Accessed February 24, 2009. http://www.nutraceuticalsworld.com/ issues/2008-11/view_features/japan/.

3. Crowther, J. (2008). China's nutraceutical industry: Market potential \& regulatory environment. Nutraceuticals World. Accessed February 24, 2009. http://dev2. nutraceuticalsworld.com/issues/2008-11/view_features/china-s-nutraceuticalindustry/.

4. Majszyk, M. (2008). Eastern Europe: The lands of emerging opportunities. Accessed February 24, 2009. http://www.nutraceuticalsworld.com/issues/2008-11/ view_features/eastern-europe/.

5. Mark, D., Abu-Rabia, A. (2008). The Middle East: Exploring the virtues of traditional Arabic Medicine. Nutraceuticals World. Accessed February 24, 2009. http:// www.nutraceuticalsworld.com/issues/2008-11/view_features/the-middle-east/.

6. Puranik, R., Dave, P.K. (2008). India: Now is the time to invest in this market. Nutraceuticals World.Accessed February 24,2009. http://www.nutraceuticalsworld. com/issues/2008-11/view_features/india/. 
7. Washington-Smith, G., Altafer, P. (2008). Latin America: Nutraceutical boom or bust? Nutraceuticals World. Accessed February 24, 2009. http://www. nutraceuticalsworld.com/issues/2008-11/view_features/latin-america/.

8. Zambetti, P. (2008). Spanning the world; Regions around the globe push full steam ahead propelled by the heat of emerging nutraceutical markets. Nutraceuticals World. Accessed February 24, 2009. http://www.nutraceuticalsworld.com/issues/ 2008-11/view_features/spanning-the-world/.

9. Gray, M.J., et al. (2009). Development of liquid chromatography/mass spectrometry methods for the quantitative analysis of herbal medicine in biological fluids: A review. Biomedical Chromatography 24(1):91-103.

10. Organization, W.H. (2008). Traditonal Medicine. World Health Organization. http://www.who.int/medicines/areas/traditional/en/.

11. Gilbert, J., Henske, P., Aingh, A. (2003). Rebuilding Big Pharma's Business Model. The Business \& Medicine Report In Vivo. 21.

12. Sollano, J.A., et al. (2008). The economics of drug discovery and the ultimate valuation of pharmacotherapies in the marketplace. Clinical Pharmacology and Therapeutics 84(2):263-266.

13. DiMasi, J.A., Hansen, R.W., Grabowski, H.G. (2003). The price of innovation: New estimates of drug development costs. Journal of Health Economics 22(2): 151-185.

14. Newman, D.J., Cragg, G.M. (2007). Natural products as sources of new drugs over the last 25 years. Journal of Natural Products 70(3):461-477.

15. Cragg, G.M., Newman, D.J., Snader, K.M. (1997). Natural products in drug discovery and development. Journal of Natural Products 60(1):52-60.

16. Khosla, C., Keasling, J.D. (2003). Metabolic engineering for drug discovery and development. Nature Reviews Drug Discovery 2(12):1019-1025.

17. Raskin, I., Ripoll, C. (2004). Can an apple a day keep the doctor away? Current Pharmaceutical Design 10(27):3419-3429.

18. Ferrer, J.L., et al. (2008). Structure and function of enzymes involved in the biosynthesis of phenylpropanoids. Plant Physiology and Biochemistry 46(3): 356-370.

19. Balandrin, M.F., et al. (1985). Natural plant chemicals: Sources of industrial and medicinal materials. Science 228(4704):1154-1160.

20. Stafford, H.A. (1990). Flavonoid Metabolism. Boca Raton, FL: CRC Press, Inc.

21. Beecher, G.R. (2003). Overview of dietary flavonoids: Nomenclature, occurrence and intake. Journal of Nutrition 133(10):3248S-3254S.

22. Bourguad, F., et al. (2001). Production of plant secondary metabolites: A historical perspective. Plant Science 161(5):839.

23. Harborne, J.B., Williams, C.A. (2000). Advances in flavonoid research since 1992. Phytochemistry 55(6):481-504.

24. Naczk, M., Shahidi, F. (2004). Extraction and analysis of phenolic in food. Journal of Chromatography. A 1054:95-111.

25. Cuyckens, F., Claeys, M. (2004). Mass spectrometry in the structural analysis of flavonoids. Journal of Mass Spectrometry 39(1):1-15. 
26. Stobiecki, M. (2000). Application of mass spectrometry for identification and structural studies of flavonoid glycosides. Phytochemistry 54(3):237-256.

27. Grundhofer, P., et al. (2001). Biosynthesis and subcellular distribution of hydrolyzable tannins. Phytochemistry 57(6):915-927.

28. Liu, E.H., et al. (2008). Advances of modern chromatographic and electrophoretic methods in separation and analysis of flavonoids. Molecules 13(10):2521-2544.

29. Barros, L., et al. (2009). Phenolic acids determination by HPLC-DAD-ESI/MS in sixteen different Portuguese wild mushrooms species. Food and Chemical Toxicology 47(6):1076-1079.

30. Hollman, P.C.H., van Trijp, J.M.P., Buysman, M.N.C.P. (1996). Fluorescence detection of flavonols in HPLC by postcolumn chelation with aluminum. Analytical Chemistry 68:3511-3515.

31. Scalbert, A., Williamson, G. (2000). Dietary intake and bioavailability of polyphenols. Journal of Nutrition 130(Suppl. 8S):2073S-2085S.

32. Vacek, J., et al. (2008). Current trends in isolation, separation, determination and identification of isoflavones: A review. Journal of Separation Science 31(11): 2054-2067.

33. Cuyckens, F., et al. (2000). Tandem mass spectral strategies for the structural characterization of flavonoid glycosides. Analusis 28(10):888-895.

34. Kodan, A., Kuroda, H., Sakai, F. (2002). A stilbene synthase from Japanese red pine (Pinus densiflora): Implications for phytoalexin accumulation and downregulation of flavonoid biosynthesis. Proceedings of the National Academy of Sciences of the United States of America 99(5):3335-3339.

35. Szent-Györgyi, A. (1936). From Vitamin C to Vitamin P. Current Science 5: 285-286.

36. Haslam, E. (1998). Practical Polyphenolics. From Structure to Molecular Recognition and Physiological Action. Cambridge, UK: Cambridge Universtiy Press.

37. Cook, N.C., Samman, S. (1996). Flavonoids-Chemistry, metabolism, cardioprotective effects, and dietary sources. The Journal of Nutritional Biochemistry 6:66-76.

38. Scalbert, A., Johnson, I.T., Saltmarsh, M. (2005). Polyphenols: Antioxidants and beyond. American Journal of Clinical Nutrition 81(Suppl. 1):215S-217S.

39. Fremont, L. (2000). Biological effects of resveratrol. Life Sciences 66(8): 663-673.

40. Rimando, A.M., et al. (2002). Cancer chemopreventive and antioxidant activities of pterostilbene, a naturally occurring analogue of resveratrol. Journal of Agricultural and Food Chemistry 50(12):3453-3457.

41. Roupe, K., et al. (2006). Pharmacometrics of stilbenes: Segueing towards the clinic. Current Clinical Pharmacology 1(1):81-101.

42. Scalbert, A., et al. (2005). Dietary polyphenols and the prevention of diseases. Critical Reviews in Food Science and Nutrition 45(4):287-306.

43. Moon, Y.J., Wang, X., Morris, M.E. (2006). Dietary flavonoids: Effects on xenobiotic and carcinogen metabolism. Toxicol In Vitro 20(2):187-210. 
44. Caristi, C., et al. (2003). Flavonoids detection by HPLC-DAD-MS-MS in lemon juices from Sicilian cultivars. Journal of Agricultural and Food Chemistry 51(12):3528-3534.

45. Kawaii, S., et al. (1999). Quantitation of flavonoid constituents in citrus fruits. Journal of Agricultural and Food Chemistry 47(9):3565-3571.

46. Nielsen, S.E., et al. (2002). Flavonoids in human urine as biomarkers for intake of fruits and vegetables. Cancer Epidemiology, Biomarkers and Prevention 11(5): 459-466.

47. Ameer, B., et al. (1996). Flavanone absorption after naringin, hesperidin, and citrus administration. Clinical Pharmacology and Therapeutics 60(1):34-40.

48. Benavente-Carcia, O., et al. (1997). Uses and properties of citrus flavonoids. Journal of Agricultural and Food Chemistry 45(12):4505-4515.

49. Brevik, A., et al. (2004). Urinary excretion of flavonoids reflects even small changes in the dietary intake of fruits and vegetables. Cancer Epidemiology, Biomarkers and Prevention 13(5):843-849.

50. Caccamese, S., Manna, L., Scivoli, G. (2003). Chiral HPLC separation and CD spectra of the C-2 diastereomers of naringin in grapefruit during maturation. Chirality 15(8):661-667.

51. Erlund, I., et al. (2001). Plasma kinetics and urinary excretion of the flavanones naringenin and hesperetin in humans after ingestion of orange juice and grapefruit juice. Journal of Nutrition 131(2):235-241.

52. Gil-Izquierdo, A., et al. (2004). Effect of the rootstock and interstock grafted in lemon tree (Citrus limon (L.) Burm.) on the flavonoid content of lemon juice. Journal of Agricultural and Food Chemistry 52(2):324-331.

53. Middleton, E., Kandaswami, C. (1994). The impact of plant flavonoids in mammalian biology: Implications for immunity, inflammation and cancer. In The Flavonoids: Advances in Research since 1986, Harborne, J., editor. London: Chapman \& Hall, pp. 619-652.

54. Miyake, Y., et al. (2006). Difference in plasma metabolite concentration after ingestion of lemon flavonoids and their aglycones in humans. Journal of Nutritional Science and Vitaminology 52(1):54-60.

55. Miyake, Y., et al. (2000). Identification and antioxidant activity of flavonoid metabolites in plasma and urine of eriocitrin-treated rats. Journal of Agricultural and Food Chemistry 48(8):3217-3224.

56. Montanari, A., Chen, J., Widmer, W. (1998). Citrus flavonoids: A review of plast biological activity against disease. Discovery of new flavonoids from Dancy tangerine cold pressed oil solids and leaves. In Flavonoids in the Living System (Advances in Experimental Medicine and Biology), Manthey, J., Buslig, B., editors. New York: Plenum, pp. 103-116.

57. Wilcox, L.J., Borradaile, N.M., Huff, M.W. (1999). Antiatherogenic properties of naringenin, a citrus flavonoid. Cardiovascular Drug Reviews 17(2):160-178.

58. Bugianesi, R., et al. (2002). Naringenin from cooked tomato paste is bioavailable in men. Journal of Nutrition 132(11):3349-3352.

59. Le Gall, G., et al. (2003). Characterization and content of flavonoid glycosides in genetically modified tomato (Lycopersicon esculentum) fruits. Journal of Agricultural and Food Chemistry 51(9):2438-2446. 
60. Stewart, A.J., et al. (2000). Occurrence of flavonols in tomatoes and tomato-based products. Journal of Agricultural and Food Chemistry 48(7):2663-2669.

61. Daigle, D.J., et al. (1988). Peanut hull flavonoids: Their relationship with peanut maturity. Journal of Agricultural and Food Chemistry 36(6):1179-1181.

62. Krause, M., Galensa, R. (1991). Analysis of enantiomeric flavanones in plant extracts by high-performance liquid chromatography on a cellulose triacetate based chiral stationary phase. Chromatographia 32(1/2):69-72.

63. Manach, C., et al. (2003). Bioavailability in humans of the flavanones hesperidin and narirutin after the ingestion of two doses of orange juice. European Journal of Clinical Nutrition 57(2):235-242.

64. Proksch, P., et al. (1984). Flavonoids from the external leaf resin of four Hemizonza species (Asteraceae). Phytochemistry 23(3):679-680.

65. Geissman, T.A. (1940). The isolation of eriodictyol and homoeriodictyol. An improved procedure. Journal of the American Chemical Society 62(11): 3258-3259.

66. van den Broucke, C.O., et al. (1982). Three methylated flavones from Thymus vulgaris. Phytochemistry 21(10):2581-2583.

67. Formica, J.V., Regelson, W. (1995). Review of the biology of quercetin and related bioflavonoids. Food and Chemical Toxicology 33(12):1061-1080.

68. Pietta, P.G. (2000). Flavonoids as antioxidants. Journal of Natural Products 63(7):1035-1042.

69. van Acker, F.A., et al. (2000). Flavonoids can replace alpha-tocopherol as an antioxidant. FEBS Letters 473(2):145-148.

70. Miyake, Y., Yamamoto, K., Osawa, T. (1997). Metabolism of antioxidant in lemon fruit (Citrus limon Burm. f.) by human intestinal bacteria. Journal of Agricultural and Food Chemistry 45(10):3738-3742.

71. Bocco, A., et al. (1998). Antioxidant activity and phenolic composition of citrus peel and seed extracts. Journal of Agricultural and Food Chemistry 46(6): 2123-2129.

72. Cao, G., Sofic, E., Prior, R.L. (1997). Antioxidant and prooxidant behavior of flavonoids: Structure-activity relationships. Free Radical Biology and Medicine 22(5):749-760.

73. Chen, J., Montanari, A.M., Widmer, W.W. (1997). Two new polymethoxylated flavones, a class of compounds with potential anticancer activity, isolated from cold pressed dancy tangerine peel oil solids. Journal of Agricultural and Food Chemistry 45(2):364-368.

74. Di Carlo, G., et al. (1999). Flavonoids: Old and new aspects of a class of natural therapeutic drugs. Life Sciences 65(4):337-353.

75. Marin, F.R., et al. (2002). Changes in nutraceutical composition of lemon juices according to different industrial extraction systems. Food Chemistry 78(3): 319-324.

76. Rice-Evans, C.A., Miller, N.J., Paganga, G. (1996). Structure-antioxidant activity relationships of flavonoids and phenolic acids. Free Radical Biology and Medicine 20(7):933-956.

77. Rusznyak, S., Szent-Györgyi, A. (1936). Vitamin P: Flavonols as vitamins. Nature 138:27. 
78. Bok, S.H., et al. (1999). Plasma and hepatic cholesterol and hepatic activities of 3-hydroxy-3-methyl-glutaryl-CoA reductase and acyl CoA: Cholesterol transferase are lower in rats fed citrus peel extract or a mixture of citrus bioflavonoids. Journal of Nutrition 129(6):1182-1185.

79. Borradaile, N.M., Carroll, K.K., Kurowska, E.M. (1999). Regulation of HepG2 cell apolipoprotein B metabolism by the citrus flavanones hesperetin and naringenin. Lipids 34(6):591-598.

80. Santos, K.F., et al. (1999). Hypolipidaemic effects of naringenin, rutin, nicotinic acid and their associations. Pharmacological Research 40(6):493-496.

81. Shin, Y.W., et al. (1999). Hypocholesterolemic effect of naringin associated with hepatic cholesterol regulating enzyme changes in rats. International Journal for Vitamin and Nutrition Research 69(5):341-347.

82. Hertog, M.G., et al. (1993). Dietary antioxidant flavonoids and risk of coronary heart disease: The Zutphen Elderly Study. Lancet 342(8878):1007-1011.

83. Kaul, T.N., Middleton, E., Jr., Ogra, P.L. (1985). Antiviral effect of flavonoids on human viruses. Journal of Medical Virology 15(1):71-79.

84. Wang, H.K., et al. (1998). Recent advances in the discovery and development of flavonoids and their analogues as antitumor and anti-HIV agents. Advances in Experimental Medicine and Biology 439:191-225.

85. Middleton, E., Jr. (1998). Effect of plant flavonoids on immune and inflammatory cell function. Advances in Experimental Medicine and Biology 439:175-182.

86. Fotsis, T., et al. (1997). Flavonoids, dietary-derived inhibitors of cell proliferation and in vitro angiogenesis. Cancer Research 57(14):2916-2921.

87. Knekt, P., et al. (1997). Dietary flavonoids and the risk of lung cancer and other malignant neoplasms. American Journal of Epidemiology 146(3):223-230.

88. So, F.V., et al. (1996). Inhibition of human breast cancer cell proliferation and delay of mammary tumorigenesis by flavonoids and citrus juices. Nutrition and Cancer 26(2):167-181.

89. Stefani, E.D., et al. (1999). Dietary antioxidants and lung cancer risk: A casecontrol study in Uruguay. Nutrition and Cancer 34(1):100-110.

90. Yang, M., et al. (1997). Chemopreventive effects of diosmin and hesperidin on N-butyl-N-(4-hydroxybutyl)nitrosamine-induced urinary-bladder carcinogenesis in male ICR mice. International Journal of Cancer 73(5):719-724.

91. Samman, S., Wall, P.M., Cook, N.C. (1999). Flavonoids and coronary heart disease: Dietary perspectives. In Flavonoids in the Living System (Advances in Experimental Medicine and Biology), Manthey, J., Buslig, B., editors. New York: Plenum Press, pp. $469-481$.

92. USDA (2003). USDA Database for the Flavonoid Content of Selected Foods. Accessed March 25, 2003 and April 9, 2007. http://www.nal.usda.gov/fnic/ foodcomp/Data/Flav/flav.html.

93. Yáñez, J.A., Andrews, P.K., Davies, N.M. (2007). Methods of analysis and separation of chiral flavonoids. Journal of Chromatography. B, Analytical Technologies in the Biomedical and Life Sciences 848(2):159-181.

94. Krause, M., Galensa, R. (1988). Direct enantiomeric separation of racemic flavanones by high-performance liquid chromatography using cellulose triacetate as a chiral stationary phase. Journal of Chromatography. A 441:417-422. 
95. Krause, M., Galensa, R. (1990). Improved chiral stationary phase based on cellulose triacetate supported on non-macroporous silica gel diol for the highperformance liquid chromatographic separation of racemic flavanones and diastereomeric flavanone glycosides. Journal of Chromatography. A 502:287-296.

96. Stafford, H.A. (1990). Biosynthesis of flavanones and 3-hydroxyflavanones (dihydroxyflavonols): The "grid" pattern of basic hydroxylations of the B- and C-rings. In Flavonoid Metabolism. Boca Raton, FL: CRC Press, Inc., pp. 39-44.

97. Hemingway, R.W., Foo, L.Y., Porter, L.J. (1982). Linkage isomerism in trimeric acid polymeric 2,3-cis procyanidins. Journal of the Chemical Society Perkin Transactions 1:1209.

98. World Health Organization. (2008). The Top Ten Causes of Death. World Health Organization. http://www.who.int/mediacentre/factsheets/fs310/en/index.html.

99. World Health Organization. (2007). The Top Ten Causes of Death. World Health Organization. http://www.who.int/mediacentre/factsheets/fs310/en/index.html.

100. National Cancer Institute. Cardiopulmonary Syndrome Overview. National Cancer Institute. http://www.cancer.gov/cancertopics/pdq/supportivecare/ cardiopulmonary/Patient/page1.

101. Niessen,W.M.A., Tinke, A.P.(1995). Liquid-chromatography mass-spectrometryGeneral principles and instrumentation. Journal of Chromatography. A 703(1-2): 37-57.

102. Singleton, V.L., Rossi, J.A., Jr. (1985). Colorimetry of total phenolics with phosphomolybdic-phosphotungstic acid reagents. American Journal of Enology and Viticulture 16(3):144-158.

103. Folin, O., Denis, W. (1912). On phosphotungstic-phosphomolybdic compounds as color reagents. Journal of Biological Chemistry 12:239-243.

104. Yáñez, J.A., et al. (2005). Stereospecific high-performance liquid chromatographic analysis of hesperetin in biological matrices. Journal of Pharmaceutical and Biomedical Analysis 37(3):591-595.

105. Garg, A., et al. (2001). Chemistry and pharmacology of the citrus bioflavonoid hesperidin. Phytotherapy Research 15(8):655-669.

106. Cvetnic, Z., Vladimir-Knezevic, S. (2004). Antimicrobial activity of grapefruit seed and pulp ethanolic extract. Acta Pharmaceutica 54(3):243-250.

107. Panasiak, W., et al. (1989). Influence of flavonoids on combined experimental infections with EMC virus and Staphylococcus aureus in mice. Acta Microbiologica Polonica 38(2):185-188.

108. Bae, E.A., Han, M.J., Kim, D.H. (1999). In vitro anti-Helicobacter pylori activity of some flavonoids and their metabolites. Planta Medica 65(5):442-443.

109. De Clercq, E. (2006). Potential antivirals and antiviral strategies against SARS coronavirus infections. Expert Review of Anti-infective Therapy 4(2):291-302.

110. Paredes, A., et al. (2003). Anti-Sindbis activity of flavanones hesperetin and naringenin. Biological and Pharmaceutical Bulletin 26(1):108-109.

111. Rotelli, A.E., et al. (2003). Comparative study of flavonoids in experimental models of inflammation. Pharmacological Research 48(6):601-606.

112. Sugishita, E., Amagaya, S., Ogihara, Y. (1981). Antiinflammatory testing methods: Comparative evaluation of mice and rats. Journal of Pharmacobio-Dynamics 4(8):565-575. 
113. Young, J.M., De Young, L.M. (1989). Cutaneous models of inflammation for the evaluation of topical and systemic pharmacological agents. In Pharmacological Methods in the Control of Inflammation, Spector, J., Back, N., editors. New York: Liss, pp. 215-231.

114. Meier, R., Schuler, W., Desaulles, P. (1950). Zur Frage des Mechanismus der Hemmung des Bindegewebswachstums durch. Cortisone Experientia VI/12(12): 469-471.

115. Mizushima, Y., Tsukada, W., Akimoto, T. (1972). A modification of rat adjuvant arthritis for testing antirheumatic drugs. Journal of Pharmacy and Pharmacology 24(10):781-785.

116. Di Rosa, M., Giroud, J.P., Willoughby, D.A. (1971). Studies on the mediators of the acute inflammatory response induced in rats in different sites by carrageenan and turpentine. Journal of Pathology 104(1):15-29.

117. Vinegar, R., Truax, J.F., Selph, J.L. (1976). Quantitative studies of the pathway to acute carrageenan inflammation. Federation Proceedings 35(13):2447-2456.

118. Vinegar, R., et al. (1982). Pathway of onset, development, and decay of carrageenan pleurisy in the rat. Federation Proceedings 41(9):2588-2595.

119. Yeh, C.C., et al. (2007). The immunomodulation of endotoxin-induced acute lung injury by hesperidin in vivo and in vitro. Life Sciences 80(20):1821-1831.

120. Cho, J. (2006). Antioxidant and neuroprotective effects of hesperidin and its aglycone hesperetin. Archives of Pharmacal Research 29(8):699-706.

121. Wilmsen, P.K., Spada, D.S., Salvador, M. (2005). Antioxidant activity of the flavonoid hesperidin in chemical and biological systems. Journal of Agricultural and Food Chemistry 53(12):4757-4761.

122. Kim, J.Y., et al. (2004). Hesperetin: A potent antioxidant against peroxynitrite. Free Radical Research 38(7):761-769.

123. Balakrishnan, A., Menon, V.P. (2007). Antioxidant properties of hesperidin in nicotine-induced lung toxicity. Fundamental and Clinical Pharmacology 21(5): 535-546.

124. Balakrishnan, A., Menon, V.P. (2007). Effect of hesperidin on matrix metalloproteinases and antioxidant status during nicotine-induced toxicity. Toxicology 238(2-3):90-98.

125. Rapavi, E., et al. (2007). The effect of citrus flavonoids on the redox state of alimentary-induced fatty liver in rats. Natural Product Research 21(3):274-281.

126. Rapavi, E., et al. (2006). Effects of citrus flavonoids on redox homeostasis of toxininjured liver in rat. Acta Biologica Hungarica 57(4):415-422.

127. Tirkey, N., et al. (2005). Hesperidin, a citrus bioflavonoid, decreases the oxidative stress produced by carbon tetrachloride in rat liver and kidney. BMC Pharmacology 5(1):2.

128. Zheng, Q., et al. (2002). Further investigation of the modifying effect of various chemopreventive agents on apoptosis and cell proliferation in human colon cancer cells. Journal of Cancer Research and Clinical Oncology 128(10): 539-546.

129. Park, H.J., et al. (2007). Apoptotic effect of hesperidin through caspase 3 activation in human colon cancer cells, SNU-C4. Phytomedicine 15(1-2):147-151. 
130. Franke, A.A., et al. (1998). Inhibition of neoplastic transformation and bioavailability of dietary flavonoid agents. Advances in Experimental Medicine and Biology 439:237-248.

131. Choi, E.J. (2007). Hesperetin induced G1-phase cell cycle arrest in human breast cancer MCF-7 cells: Involvement of CDK4 and p21. Nutrition and Cancer 59(1):115-119.

132. Corpet, D.E., Tache, S. (2002). Most effective colon cancer chemopreventive agents in rats: A systematic review of aberrant crypt foci and tumor data, ranked by potency. Nutrition and Cancer 43(1):1-21.

133. Corpet, D.E., Pierre, F. (2003). Point: From animal models to prevention of colon cancer. Systematic review of chemoprevention in min mice and choice of the model system. Cancer Epidemiology, Biomarkers and Prevention 12(5): 391-400.

134. Franke, A.A., et al. (2002). Inhibition of colonic aberrant crypt formation by the dietary flavonoids (+)-catechin and hesperidin. Advances in Experimental Medicine and Biology 505:123-133.

135. Tanaka, T., et al. (2000). Suppression of azoxymethane-induced colon carcinogenesis in male F344 rats by mandarin juices rich in beta-cryptoxanthin and hesperidin. International Journal of Cancer 88(1):146-150.

136. Miyagi, Y., et al. (2000). Inhibition of azoxymethane-induced colon cancer by orange juice. Nutrition and Cancer 36(2):224-229.

137. Berkarda, B., et al. (1998). Inhibitory effect of hesperidin on tumour initiation and promotion in mouse skin. Research in Experimental Medicine 198(2):93-99.

138. Tanaka, T., et al. (1997). Modulation of N-methyl-N-amylnitrosamine-induced rat oesophageal tumourigenesis by dietary feeding of diosmin and hesperidin, both alone and in combination. Carcinogenesis 18(4):761-769.

139. Sakata, K., et al. (2003). Inhibition of inducible isoforms of cyclooxygenase and nitric oxide synthase by flavonoid hesperidin in mouse macrophage cell line. Cancer Letters 199(2):139-145.

140. Hirata, A., et al. (2005). Kinetics of radical-scavenging activity of hesperetin and hesperidin and their inhibitory activity on COX-2 expression. Anticancer Research 25(5):3367-3374.

141. Hsu, C.L., Yen, G.C. (2006). Induction of cell apoptosis in 3T3-L1 pre-adipocytes by flavonoids is associated with their antioxidant activity. Molecular Nutrition and Food Research 50(11):1072-1079.

142. Hsu, C.L., Yen, G.C. (2007). Effects of flavonoids and phenolic acids on the inhibition of adipogenesis in 3T3-L1 adipocytes. Journal of Agricultural and Food Chemistry 55(21):8404-8410.

143. Philp, H.A. (2003). Hot flashes-A review of the literature on alternative and complementary treatment approaches. Alternative Medicine Review 8(3): 284-302.

144. Klinge, C.M., et al. (2003). Estrogenic activity in white and red wine extracts. Journal of Agricultural and Food Chemistry 51(7):1850-1857.

145. Smith, C.J. (1964). Non-hormonal control of vaso-motor flushing in menopausal patients. Chicago Medicine 67:193-195. 
146. Ho, P.C., et al. (2000). Content of CYP3A4 inhibitors, naringin, naringenin and bergapten in grapefruit and grapefruit juice products. Pharmaceutica Acta Helvetiae 74(4):379-385.

147. Exarchou, V., et al. (2003). LC-UV-solid-phase extraction-NMR-MS combined with a cryogenic flow probe and its application to the identification of compounds present in Greek oregano. Analytical Chemistry 75(22):6288-6294.

148. Minoggio, M., et al. (2003). Polyphenol pattern and antioxidant activity of different tomato lines and cultivars. Annals of Nutrition and Metabolism 47(2):64-69.

149. Sanchez-Rabaneda, F., et al. (2003). Liquid chromatographic/electrospray ionization tandem mass spectrometric study of the phenolic composition of cocoa (Theobroma cacao). Journal of Mass Spectrometry 38(1):35-42.

150. Wang, H., et al. (1999). Antioxidant polyphenols from tart cherries (Prunus cerasus). Journal of Agricultural and Food Chemistry 47(3):840-844.

151. Hungria, M., Johnston, A.W., Phillips, D.A. (1992). Effects of flavonoids released naturally from bean (Phaseolus vulgaris) on nodD-regulated gene transcription in Rhizobium leguminosarum bv. phaseoli. Molecular Plant-Microbe Interactions 5(3):199-203.

152. El-Gammal, A.A., Mansour, R.M. (1986). Antimicrobial activities of some flavonoid compounds. Zentralblatt fur Mikrobiologie 141(7):561-565.

153. Tereschuk, M.L., et al. (2004). Flavonoids from Argentine Tagetes (Asteraceae) with antimicrobial activity. Methods in Molecular Biology 268:317-330.

154. Ramesh, N., et al. (2001). Phytochemical and antimicrobial studies on Drynaria quercifolia. Fitoterapia 72(8):934-936.

155. Koru, O., et al. (2007). In vitro antimicrobial activity of propolis samples from different geographical origins against certain oral pathogens. Anaerobe 13(3-4):140-145.

156. Uzel, A., et al. (2005). Chemical compositions and antimicrobial activities of four different Anatolian propolis samples. Microbiological Research 160(2):189-195.

157. Suzgec, S., et al. (2005). Flavonoids of Helichrysum compactum and their antioxidant and antibacterial activity. Fitoterapia 76(2):269-272.

158. Lyu, S.Y., Rhim, J.Y., Park, W.B. (2005). Antiherpetic activities of flavonoids against herpes simplex virus type 1 (HSV-1) and type 2 (HSV-2) in vitro. Archives of Pharmacal Research 28(11):1293-1301.

159. Castrillo, J.L., Vanden Berghe, D., Carrasco, L. (1986). 3-Methylquercetin is a potent and selective inhibitor of poliovirus RNA synthesis. Virology 152(1): 219-227.

160. Lotito, S.B., Frei, B. (2006). Dietary flavonoids attenuate tumor necrosis factor alpha-induced adhesion molecule expression in human aortic endothelial cells. Structure-function relationships and activity after first pass metabolism. Journal of Biological Chemistry 281(48):37102-37110.

161. Hougee, S., et al. (2005). Decreased pro-inflammatory cytokine production by LPS-stimulated PBMC upon in vitro incubation with the flavonoids apigenin, luteolin or chrysin, due to selective elimination of monocytes/macrophages. Biochemical Pharmacology 69(2):241-248.

162. Olszanecki, R., et al. (2002). Flavonoids and nitric oxide synthase. Journal of Physiology and Pharmacology 53(4 Pt 1):571-584. 
163. Kanno, S., et al. (2006). Inhibitory effect of naringin on lipopolysaccharide (LPS)induced endotoxin shock in mice and nitric oxide production in RAW 264.7 macrophages. Life Sciences 78(7):673-681.

164. Kawaguchi, K., et al. (2004). Suppression of infection-induced endotoxin shock in mice by a citrus flavanone naringin. Planta Medica 70(1):17-22.

165. Rajadurai, M., Prince, P.S. (2007). Preventive effect of naringin on isoproterenolinduced cardiotoxicity in Wistar rats: An in vivo and in vitro study. Toxicology 232(3):216-225.

166. Yu, J., et al. (2005). Antioxidant activity of citrus limonoids, flavonoids, and coumarins. Journal of Agricultural and Food Chemistry 53(6):2009-2014.

167. Jagetia, G.C., et al. (2004). Influence of naringin on ferric iron induced oxidative damage in vitro. Clinica Chimica Acta 347(1-2):189-197.

168. Gorinstein, S., et al. (2005). Changes in plasma lipid and antioxidant activity in rats as a result of naringin and red grapefruit supplementation. Journal of Agricultural and Food Chemistry 53(8):3223-3228.

169. Kanno, S., et al. (2005). Inhibitory effects of naringenin on tumor growth in human cancer cell lines and sarcoma S-180-implanted mice. Biological and Pharmaceutical Bulletin 28(3):527-530.

170. Frydoonfar, H.R., McGrath, D.R., Spigelman, A.D. (2003). The variable effect on proliferation of a colon cancer cell line by the citrus fruit flavonoid naringenin. Colorectal Disease 5(2):149-152.

171. Kanno, S., et al. (2004). Effects of naringin on cytosine arabinoside (Ara-C)induced cytotoxicity and apoptosis in P388 cells. Life Sciences 75(3):353-365.

172. van Meeuwen, J.A., et al. (2007). (Anti)estrogenic effects of phytochemicals on human primary mammary fibroblasts, MCF-7 cells and their co-culture. Toxicology and Applied Pharmacology 221(3):372-383.

173. Totta, P., et al. (2004). Mechanisms of naringenin-induced apoptotic cascade in cancer cells: Involvement of estrogen receptor alpha and beta signalling. IUBMB Life 56(8):491-499.

174. Harmon, A.W., Patel, Y.M. (2004). Naringenin inhibits glucose uptake in MCF-7 breast cancer cells: A mechanism for impaired cellular proliferation. Breast Cancer Research and Treatment 85(2):103-110.

175. Gopalakrishnan, A., et al. (2006). Modulation of activator protein-1 (AP-1) and MAPK pathway by flavonoids in human prostate cancer PC3 cells. Archives of Pharmacal Research 29(8):633-644.

176. Kanno, S., et al. (2006). Naringenin-induced apoptosis via activation of NF-kappaB and necrosis involving the loss of ATP in human promyeloleukemia HL-60 cells. Toxicology Letters 166(2):131-139.

177. Katula, K.S., McCain, J.A., Radewicz, A.T. (2005). Relative ability of dietary compounds to modulate nuclear factor-kappaB activity as assessed in a cell-based reporter system. Journal of Medicinal Food 8(2):269-274.

178. Schindler, R., Mentlein, R. (2006). Flavonoids and vitamin E reduce the release of the angiogenic peptide vascular endothelial growth factor from human tumor cells. Journal of Nutrition 136(6):1477-1482.

179. Vanamala, J., et al. (2006). Suppression of colon carcinogenesis by bioactive compounds in grapefruit. Carcinogenesis 27(6):1257-1265. 
180. Raso, G.M., et al. (2001). Inhibition of inducible nitric oxide synthase and cyclooxygenase-2 expression by flavonoids in macrophage J774A.1. Life Sciences 68(8):921-931.

181. Rajadurai, M., Stanely Mainzen Prince, P. (2007). Preventive effect of naringin on cardiac markers, electrocardiographic patterns and lysosomal hydrolases in normal and isoproterenol-induced myocardial infarction in Wistar rats. Toxicology 230(2-3):178-188.

182. Arayne, M.S., Sultana, N., Bibi, Z. (2005). Grape fruit juice-drug interactions. Pakistan Journal of Pharmaceutical Sciences 18(4):45-57.

183. Bailey, D.G., et al. (2000). Grapefruit-felodipine interaction: Effect of unprocessed fruit and probable active ingredients. Clinical Pharmacology and Therapeutics 68(5):468-477.

184. Eagling, V.A., Profit, L., Back, D.J. (1999). Inhibition of the CYP3A4-mediated metabolism and P-glycoprotein-mediated transport of the HIV-1 protease inhibitor saquinavir by grapefruit juice components. British Journal of Clinical Pharmacology 48(4):543-552.

185. Zhang, H., et al. (2000). Effect of the grapefruit flavonoid naringin on pharmacokinetics of quinine in rats. Drug Metabolism and Drug Interactions 17(1-4): 351-363.

186. Yeum, C.H., Choi, J.S. (2006). Effect of naringin pretreatment on bioavailability of verapamil in rabbits. Archives of Pharmacal Research 29(1):102-107.

187. Kim, H.J., Choi, J.S. (2005). Effects of naringin on the pharmacokinetics of verapamil and one of its metabolites, norverapamil, in rabbits. Biopharmaceutics and Drug Disposition 26(7):295-300.

188. Guo, L.Q., et al. (2000). Role of furanocoumarin derivatives on grapefruit juicemediated inhibition of human CYP3A activity. Drug Metabolism and Disposition: The Biological Fate of Chemicals 28(7):766-771.

189. Guo, L.Q., et al. (2000). Inhibitory effect of natural furanocoumarins on human microsomal cytochrome P450 3A activity. Japanese Journal of Pharmacology 82(2):122-129.

190. Chan, W.K., et al. (1998). Mechanism-based inactivation of human cytochrome P450 3A4 by grapefruit juice and red wine. Life Sciences 62(10):PL135-PL142.

191. Runkel, M., et al. (1997). The character of inhibition of the metabolism of 1,2-benzopyrone (coumarin) by grapefruit juice in human. European Journal of Clinical Pharmacology 53(3-4):265-269.

192. Bailey, D.G., et al. (1993). Effect of grapefruit juice and naringin on nisoldipine pharmacokinetics. Clinical Pharmacology and Therapeutics 54(6):589-594.

193. Bailey, D.G., et al. (1993). Grapefruit juice-felodipine interaction: Mechanism, predictability, and effect of naringin. Clinical Pharmacology and Therapeutics 53(6):637-642.

194. Edwards, D.J., Bernier, S.M. (1996). Naringin and naringenin are not the primary CYP3A inhibitors in grapefruit juice. Life Sciences 59:1025-1030.

195. Guengerich, F.P., Kim, D.H. (1990). In vitro inhibition of dihydropyridine oxidation and aflatoxin B1 activation in human liver microsomes by naringenin and other flavonoids. Carcinogenesis 11(12):2275-2279. 
196. Miniscalco, A., et al. (1992). Inhibition of dihydropyridine metabolism in rat and human liver microsomes by flavonoids found in grapefruit juice. Journal of Pharmacology and Experimental Therapeutics 261(3):1195-1199.

197. Edwards, D.J., Bellevue, F.H., 3rd, Woster, P.M. (1996). Identification of 6',7'-dihydroxybergamottin, a cytochrome P450 inhibitor, in grapefruit juice. Drug Metabolism and Disposition: The Biological Fate of Chemicals 24(12):1287-1290.

198. Schmiedlin-Ren, P., et al. (1997). Mechanisms of enhanced oral availability of CYP3A4 substrates by grapefruit constituents. Decreased enterocyte CYP3A4 concentration and mechanism-based inactivation by furanocoumarins. Drug Metabolism and Disposition: The Biological Fate of Chemicals 25(11): 1228-1233.

199. Fukuda, K., Ohta, T., Yamazoe, Y. (1997). Grapefruit component interacting with rat and human P450 CYP3A: Possible involvement of non-flavonoid components in drug interaction. Biological and Pharmaceutical Bulletin 20(5):560-564.

200. Ameer, B., Weintraub, R.A. (1997). Drug interactions with grapefruit juice. Clinical Pharmacokinetics 33(2):103-121.

201. Fuhr, U. (1998). Drug interactions with grapefruit juice. Extent, probable mechanism and clinical relevance. Drug Safety 18(4):251-272.

202. Bailey, D.G., et al. (1998). Grapefruit juice-drug interactions. British Journal of Clinical Pharmacology 46(2):101-110.

203. Lown, K.S., et al. (1997). Grapefruit juice increases felodipine oral availability in humans by decreasing intestinal CYP3A protein expression. Journal of Clinical Investigation 99(10):2545-2553.

204. Soldner, A., et al. (1999). Grapefruit juice activates P-glycoprotein-mediated drug transport. Pharmaceutical Research 16(4):478-485.

205. Grundy, J.S., et al. (1998). Grapefruit juice and orange juice effects on the bioavailability of nifedipine in the rat. Biopharmaceutics and Drug Disposition 19(3): 175-183.

206. Hashimoto, K., et al. (1998). Interaction of citrus juices with pranidipine, a new 1,4-dihydropyridine calcium antagonist, in healthy subjects. European Journal of Clinical Pharmacology 54(9-10):753-760.

207. Jagetia, A., Jagetia, G.C., Jha, S. (2007). Naringin, a grapefruit flavanone, protects V79 cells against the bleomycin-induced genotoxicity and decline in survival. Journal of Applied Toxicology 27(2):122-132.

208. Jagetia, G.C., Venkatesha, V.A. (2006). Treatment of mice with stem bark extract of Aphanamixis polystachya reduces radiation-induced chromosome damage. International Journal of Radiation Biology 82(3):197-209.

209. Sudjaroen, Y., et al. (2005). Isolation and structure elucidation of phenolic antioxidants from tamarind (Tamarindus indica L.) seeds and pericarp. Food and Chemical Toxicology 43(11):1673-1682.

210. Kosar, M., et al. (2004). Screening of free radical scavenging compounds in water extracts of Mentha samples using a postcolumn derivatization method. Journal of Agricultural and Food Chemistry 52(16):5004-5010.

211. Parejo, I., et al. (2004). Bioguided isolation and identification of the nonvolatile antioxidant compounds from fennel (Foeniculum vulgare Mill.) waste. Journal of Agricultural and Food Chemistry 52(7):1890-1897. 
212. Dapkevicius, A., et al. (2002). Isolation and structure elucidation of radical scavengers from Thymus vulgaris leaves. Journal of Natural Products 65(6): 892-896.

213. Hvattum, E. (2002). Determination of phenolic compounds in rose hip (Rosa canina) using liquid chromatography coupled to electrospray ionisation tandem mass spectrometry and diode-array detection. Rapid Communications in Mass Spectrometry 16(7):655-662.

214. McKay, D.L., Blumberg, J.B. (2006). A review of the bioactivity and potential health benefits of peppermint tea (Mentha piperita L.). Phytotherapy Research 20(8):619-633.

215. Ahmed, M.S., et al. (2001). A weakly antimalarial biflavanone from Rhus retinorrhoea. Phytochemistry 58(4):599-602.

216. Zhou, L., et al. (2007). Antibacterial phenolic compounds from the spines of Gleditsia sinensis Lam. Natural Product Research 21(4):283-291.

217. Ismaili, H., et al. (2002). Topical antiinflammatory activity of extracts and compounds from Thymus broussonettii. Journal of Pharmacy and Pharmacology 54(8):1137-1140.

218. Xagorari, A., et al. (2001). Luteolin inhibits an endotoxin-stimulated phosphorylation cascade and proinflammatory cytokine production in macrophages. Journal of Pharmacology and Experimental Therapeutics 296(1):181-187.

219. Miyake, Y., et al. (2007). Isolation of antioxidative phenolic glucosides from lemon juice and their suppressive effect on the expression of blood adhesion molecules. Bioscience, Biotechnology, and Biochemistry 71(8):1911-1919.

220. Sroka, Z., Fecka, I., Cisowski, W. (2005). Antiradical and anti- $\mathrm{H}_{2} \mathrm{O}_{2}$ properties of polyphenolic compounds from an aqueous peppermint extract. Zeitschrift fur Naturforschung. Section C. Biosciences 60(11-12):826-832.

221. Miyake, Y., et al. (1998). Protective effects of lemon flavonoids on oxidative stress in diabetic rats. Lipids 33(7):689-695.

222. Narvaez-Mastache, J.M., Novillo, F., Delgado, G. (2007). Antioxidant arylprenylcoumarin, flavan-3-ols and flavonoids from Eysenhardtia subcoriacea. Phytochemistry 69(2):451-456.

223. Edenharder, R., Grunhage, D. (2003). Free radical scavenging abilities of flavonoids as mechanism of protection against mutagenicity induced by tert-butyl hydroperoxide or cumene hydroperoxide in Salmonella typhimurium TA102. Mutation Research 540(1):1-18.

224. Lee, E.R., et al. (2007). The anti-apoptotic and anti-oxidant effect of eriodictyol on UV-induced apoptosis in keratinocytes. Biological and Pharmaceutical Bulletin 30(1):32-37.

225. Ogata, S., et al. (2000). Apoptosis induced by the flavonoid from lemon fruit (Citrus limon Burm. f.) and its metabolites in HL-60 cells. Bioscience, Biotechnology, and Biochemistry 64(5):1075-1078.

226. Habtemariam, S. (1997). Flavonoids as inhibitors or enhancers of the cytotoxicity of tumor necrosis factor-alpha in L-929 tumor cells. Journal of Natural Products 60(8):775-778.

227. Kawaii, S., et al. (1999). Antiproliferative activity of flavonoids on several cancer cell lines. Bioscience, Biotechnology, and Biochemistry 63(5):896-899. 
228. Zhang, X., et al. (2006). Antiinflammatory activity of flavonoids from Populus davidiana. Archives of Pharmacal Research 29(12):1102-1108.

229. Cuendet, M., et al. (2000). A stilbene and dihydrochalcones with radical scavenging activities from Loiseleuria procumbens. Phytochemistry 54(8):871-874.

230. Escarpa, A., Gonzalez, M.C. (1998). High-performance liquid chromatography with diode-array detection for the determination of phenolic compounds in peel and pulp from different apple varieties. Journal of Chromatography. A 823(1-2): 331-337.

231. Mancini, S.D., Edwards, J.M. (1979). Cytotoxic principles from the sap of Kalmia latifolia. Journal of Natural Products 42(5):483-488.

232. Tsao, R., et al. (2003). Polyphenolic profiles in eight apple cultivars using highperformance liquid chromatography (HPLC). Journal of Agricultural and Food Chemistry 51(21):6347-6353.

233. Yao, G.M., et al. (2005). Dihydrochalcones from the leaves of Pieris japonica. Journal of Natural Products 68(3):392-396.

234. An, R.B., et al. (2007). Cytoprotective constituent of Hoveniae lignum on both Hep G2 cells and rat primary hepatocytes. Archives of Pharmacal Research 30(6):674-677.

235. Ehrenkranz, J.R., et al. (2005). Phlorizin: A review. Diabetes/Metabolism Research and Reviews 21(1):31-38.

236. Hilt, P., et al. (2003). Detection of phloridzin in strawberries (Fragaria $\times$ ananassa Duch.) by HPLC-PDA-MS/MS and NMR spectroscopy. Journal of Agricultural and Food Chemistry 51(10):2896-2899.

237. Yang, W.M., et al. (2004). Antioxidant activities of three dihydrochalcone glucosides from leaves of Lithocarpus pachyphyllus. Zeitschrift fur Naturforschung. Section C. Biosciences 59(7-8):481-484.

238. Yáñez, J.A., Davies, N.M. (2005). Stereospecific high-performance liquid chromatographic analysis of naringenin in urine. Journal of Pharmaceutical and Biomedical Analysis 39(1-2):164-169.

239. McKay, D.L., Blumberg, J.B. (2007). A review of the bioactivity of South African herbal teas: Rooibos (Aspalathus linearis) and honeybush (Cyclopia intermedia). Phytotherapy Research 21(1):1-16.

240. Marnewick, J.L., Gelderblom, W.C., Joubert, E. (2000). An investigation on the antimutagenic properties of South African herbal teas. Mutation Research 471(1-2):157-166.

241. Ulicna, O., et al. (2003). Hepatoprotective effect of rooibos tea (Aspalathus linearis) on CCl4-induced liver damage in rats. Physiological Research 52(4): 461-466.

242. Kawano, A., et al. (2009). Hypoglycemic effect of aspalathin, a rooibos tea component from Aspalathus linearis, in type 2 diabetic model db/db mice. Phytomedicine 16(5):437-443.

243. van der Merwe, J.D., et al. (2009). In vitro hepatic biotransformation of aspalathin and nothofagin, dihydrochalcones of rooibos (Aspalathus linearis), and assessment of metabolite antioxidant activity. Journal of Agricultural and Food Chemistry 58(4):2214-2220. 
244. Snijman, P.W., et al. (2009). Antioxidant activity of the dihydrochalcones aspalathin and nothofagin and their corresponding flavones in relation to other rooibos (Aspalathus linearis) flavonoids, epigallocatechin gallate, and Trolox. Journal of Agricultural and Food Chemistry 57(15):6678-6684.

245. Snijman, P.W., et al. (2007). The antimutagenic activity of the major flavonoids of rooibos (Aspalathus linearis): Some dose-response effects on mutagen activationflavonoid interactions. Mutation Research 631(2):111-123.

246. Rezk, B.M., et al. (2002). The antioxidant activity of phloretin: The disclosure of a new antioxidant pharmacophore in flavonoids. Biochemical and Biophysical Research Communications 295(1):9-13.

247. Nakamura, Y., et al. (2003). Dihydrochalcones: Evaluation as novel radical scavenging antioxidants. Journal of Agricultural and Food Chemistry 51(11): 3309-3312.

248. Nelson, J.A., Falk, R.E. (1993). The efficacy of phloridzin and phloretin on tumor cell growth. Anticancer Research 13(6A):2287-2292.

249. Nelson, J.A., Falk, R.E. (1993). Phloridzin and phloretin inhibition of 2-deoxyD-glucose uptake by tumor cells in vitro and in vivo. Anticancer Research 13(6A):2293-2299.

250. Kobori, M., et al. (1997). Phloretin-induced apoptosis in B16 melanoma 4A5 cells by inhibition of glucose transmembrane transport. Cancer Letters 119(2): 207-212.

251. Molnar, J., et al. (2010). Reversal of multidrug resistance by natural substances from plants. Current Topics in Medicinal Chemistry 10(17):1757-1768.

252. Nguyen, H., Zhang, S., Morris, M.E. (2003). Effect of flavonoids on MRP1mediated transport in Panc-1 cells. Journal of Pharmaceutical Sciences 92(2): 250-257.

253. Yang, K.C., et al. (2009). Apple polyphenol phloretin potentiates the anticancer actions of paclitaxel through induction of apoptosis in human hep G2 cells. Molecular Carcinogenesis 48(5):420-431.

254. Wang, J., et al. (2010). Estrogenic and antiestrogenic activities of phloridzin. Biological and Pharmaceutical Bulletin 33(4):592-597.

255. Gould, G.W., Holman, G.D. (1993). The glucose transporter family: Structure, function and tissue-specific expression. Biochemical Journal 295(Pt 2):329-341.

256. Chen, C.H., et al. (2007). Interaction of flavonoids and intestinal facilitated glucose transporters. Planta Medica 73(4):348-354.

257. Boyer, J., Liu, R.H. (2004). Apple phytochemicals and their health benefits. Nutrition Journal 3:5.

258. Kellett, G.L., et al. (2008). Sugar absorption in the intestine: The role of GLUT2. Annual Review of Nutrition 28:35-54.

259. Kellett, G.L., Brot-Laroche, E. (2005). Apical GLUT2: A major pathway of intestinal sugar absorption. Diabetes 54(10):3056-3062.

260. Kwon, O., et al. (2007). Inhibition of the intestinal glucose transporter GLUT2 by flavonoids. FASEB Journal 21(2):366-377.

261. Manzano, S., Williamson, G. (2010). Polyphenols and phenolic acids from strawberry and apple decrease glucose uptake and transport by human intestinal Caco2 cells. Molecular Nutrition and Food Research 54(12):1773-1780. 
262. Stangl, V., et al. (2005). The flavonoid phloretin suppresses stimulated expression of endothelial adhesion molecules and reduces activation of human platelets. Journal of Nutrition 135(2):172-178.

263. Kobori, M., et al. (1999). Phloretin-induced apoptosis in B16 melanoma 4A5 cells and HL60 human leukemia cells. Bioscience, Biotechnology, and Biochemistry 63(4):719-725.

264. Park, S.Y., et al. (2007). Induction of apoptosis in HT-29 colon cancer cells by phloretin. Journal of Medicinal Food 10(4):581-586.

265. Kim, M.S., et al. (2009). Phloretin induces apoptosis in H-Ras MCF10A human breast tumor cells through the activation of p53 via JNK and p38 mitogenactivated protein kinase signaling. Annals of the New York Academy of Sciences 1171:479-483.

266. Zhang, S., et al. (2010). Interactions between the flavonoid biochanin A and P-glycoprotein substrates in rats: In vitro and in vivo. Journal of Pharmaceutical Sciences 99(1):430-441.

267. Zhang, S., Morris, M.E. (2003). Effects of the flavonoids biochanin A, morin, phloretin, and silymarin on P-glycoprotein-mediated transport. Journal of Pharmacology and Experimental Therapeutics 304(3):1258-1267.

268. Jung, M., et al. (2009). Influence of apple polyphenols on inflammatory gene expression. Molecular Nutrition and Food Research 53(10):1263-1280.

269. Lu, X.Y., et al. (2009). Antiinflammatory and immunosuppressive effect of phloretin. Yао Хие Хие Вао 44(5):480-485.

270. Pohl, C., et al. (2006). Cytochrome P450 1A1 expression and activity in Caco-2 cells: Modulation by apple juice extract and certain apple polyphenols. Journal of Agricultural and Food Chemistry 54(26):10262-10268.

271. Mossa, J.S., et al. (1996). Free Flavonoids from Rhus retinorrhoea Steud ex Olive. Pharmaceutical Biology 34(3):198-201.

272. Grael, C.F., et al. (2000). A study of the trypanocidal and analgesic properties from Lychnophora granmongolense (Duarte) Semir \& Leitao Filho. Phytotherapy Research 14(3):203-206.

273. Ley, J.P., et al. (2005). Evaluation of bitter masking flavanones from yerba santa (Eriodictyon californicum (H. and A.) Torr., Hydrophyllaceae). Journal of Agricultural and Food Chemistry 53(15):6061-6066.

274. Lin, J.H., Chiou, Y.N., Lin, Y.L. (2002). Phenolic glycosides from Viscum angulatum. Journal of Natural Products 65(5):638-640.

275. Yin, J., et al. (2008). Inhibitory activity of the ethyl acetate fraction from Viscum coloratum on bone resorption. Planta Medica 74(2):120-125.

276. Zhao, Y., et al. (2007). HPLC determination and pharmacokinetic study of homoeriodictyol-7-O-beta-D-glucopyranoside in rat plasma and tissues. Biological and Pharmaceutical Bulletin 30(4):617-620.

277. Fukunaga, T., et al. (1989). Studies on the constituents of Japanese mistletoe, Viscum album L. var. coloratum OHWI grown on different host trees. Chemical and Pharmaceutical Bulletin 37(5):1300-1303.

278. McCormick, S. (1978). Pigment synthesis in maize aleurone from precursors fed to anthocyanin mutants. Biochemical Genetics 16(7-8):777-785. 
279. Ibrahim, A.R., et al. (2003). O-demethylation and sulfation of 7-methoxylated flavanones by Cunninghamella elegans. Chemical and Pharmaceutical Bulletin 51(2):203-206.

280. Schröder, G., et al. (2004). Flavonoid methylation: A novel 4'-O-methyltransferase from Catharanthus roseus, and evidence that partially methylated flavanones are substrates of four different flavonoid dioxygenases. Phytochemistry 65(8): 1085-1094.

281. Matsumoto, H., et al. (2004). Identification and quantification of the conjugated metabolites derived from orally administered hesperidin in rat plasma. Journal of Agricultural and Food Chemistry 52(21):6653-6659.

282. Booth, A.N., Jones, F.T., De, E.F. (1958). Metabolic fate of hesperidin, eriodictyol, homoeridictyol, and diosmin. Journal of Biological Chemistry 230(2):661-668.

283. Zhao, Y., et al. (2007). Simultaneous determination of homoeriodictyol-7-O-betaD-glucopyranoside and its metabolite homoeriodictyol in rat tissues and urine by liquid chromatography-mass spectrometry. Journal of Pharmaceutical and Biomedical Analysis 44(1):293-300.

284. Doostdar, H., Burke, M.D., Mayer, R.T. (2000). Bioflavonoids: Selective substrates and inhibitors for cytochrome P450 CYP1A and CYP1B1. Toxicology 144(1-3): 31-38.

285. Liu, Y.L., et al. (1992). Isolation of potential cancer chemopreventive agents from Eriodictyon californicum. Journal of Natural Products 55(3):357-363.

286. Ley, J.P., et al. (2008). Structural analogues of homoeriodictyol as flavor modifiers. Part III: Short chain gingerdione derivatives. Journal of Agricultural and Food Chemistry 56(15):6656-6664.

287. Shimizu, K., et al. (2004). Antioxidative bioavailability of artepillin C in Brazilian propolis. Archives of Biochemistry and Biophysics 424(2):181-188.

288. Simoes, L.M., et al. (2004). Effect of Brazilian green propolis on the production of reactive oxygen species by stimulated neutrophils. Journal of Ethnopharmacology 94(1):59-65.

289. Tavares, D.C., et al. (2007). Effects of propolis crude hydroalcoholic extract on chromosomal aberrations induced by Doxorubicin in rats. Planta Medica 73(15):1531-1536.

290. de Sousa, J.P., et al. (2007). A reliable quantitative method for the analysis of phenolic compounds in Brazilian propolis by reverse phase high performance liquid chromatography. Journal of Separation Science 30(16):2656-2665.

291. Missima, F., et al. (2007). Effect of Baccharis dracunculifolia D.C. (Asteraceae) extracts and its isolated compounds on macrophage activation. Journal of Pharmacy and Pharmacology 59(3):463-468.

292. Garcez, F.R., et al. (2006). Bioactive flavonoids and triterpenes from Terminalia fagifolia (Combretaceae). Journal of Brazilian Chemical Society 17(7):12231228.

293. Suksamrarn, A., et al. (2004). Antimycobacterial activity and cytotoxicity of flavonoids from the flowers of Chromolaena odorata. Archives of Pharmacal Research 27(5):507-511.

294. Metwally, A.M., Ekejiuba, E.C. (1981). Methoxylated Flavonols and flavanones from Eupatorium odoratum. Planta Medica 42(8):403-405. 
295. Vanamala, J., et al. (2004). Variation in the content of bioactive flavonoids in different brands of orange and grapefruit juices. Journal of Food Composition and Analysis 19:157-166.

296. Anagnostopoulou, M.A., et al. (2005). Analysis of antioxidant compounds in sweet orange peel by HPLC-diode array detection-electrospray ionization mass spectrometry. Biomedical Chromatography 19(2):138-148.

297. Calabro, M.L., et al. (2004). Study of the extraction procedure by experimental design and validation of a LC method for determination of flavonoids in Citrus bergamia juice. Journal of Pharmaceutical and Biomedical Analysis 35(2): 349-363.

298. Ross, S.A., et al. (2000). Variance of common flavonoids by brand of grapefruit juice. Fitoterapia 71(2):154-161.

299. Cancalon, P.F. (1999). Analytical monitoring of citrus juices by using capillary electrophoresis. Journal of AOAC International 82(1):95-106.

300. Avula, B., et al. (2005). Liquid chromatography of separation and quantitative determination of adrenergic amines and flavonoids from Poncirus trifoliatus Raf. fruits at different stages of growth. Chromatographia 62(7/8):379-383.

301. Han, A.R., et al. (2007). A new flavanone glycoside from the dried immature fruits of Poncirus trifoliata. Chemical and Pharmaceutical Bulletin 55(8):1270-1273.

302. Kim, C.Y., et al. (2007). One step purification of flavanone glycosides from Poncirus trifoliata by centrifugal partition chromatography. Journal of Separation Science 30(16):2693-2697.

303. Kim, D.H., Bae, E.A., Han, M.J. (1999). Anti-Helicobacter pylori activity of the metabolites of poncirin from Poncirus trifoliata by human intestinal bacteria. Biological and Pharmaceutical Bulletin 22(4):422-424.

304. Kim, J.B., et al. (2007). Inhibition of LPS-induced iNOS, COX-2 and cytokines expression by poncirin through the NF-kappaB inactivation in RAW 264.7 macrophage cells. Biological and Pharmaceutical Bulletin 30(12):2345-2351.

305. Park, S.H., Park, E.K., Kim, D.H. (2005). Passive cutaneous anaphylaxis-inhibitory activity of flavanones from Citrus unshiu and Poncirus trifoliata. Planta Medica 71(1):24-27.

306. Horowitz, R.M., Gentili, B. (1977). Flavonoid constituents of citrus. In Citrus Science and Technology, vol. 1, Nagy, S., Shaw, P.E., Veldhius, M.K., editors. Westport, CT: The Avi Publishing Company, Inc., p. 529.

307. Kim, D.H., et al. (2005). Regiospecific methylation of naringenin to ponciretin by soybean O-methyltransferase expressed in Escherichia coli.Journal of Biotechnology 119(2):155-162.

308. Shimokoriyama, M. (1956). Interconversion of chalcones and flavanones of a phloroglucinol-type structure. Journal of the American Chemical Society 79: 4199-4202.

309. Silberberg, M., et al. (2006). Flavanone metabolism in healthy and tumor-bearing rats. Biomedicine and Pharmacotherapy 60(9):529-535.

310. Lee, D.S., et al. (2002). Fecal metabolic activities of herbal components to bioactive compounds. Archives of Pharmacal Research 25(2):165-169.

311. Kim, D.H., et al. (1998). Intestinal bacterial metabolism of flavonoids and its relation to some biological activities. Archives of Pharmacal Research 21(1):17-23. 
312. Sacco, S., Maffei, M. (1997). The effect of isosakuranetin (5,7-dihydroxy 4'-methoxy flavanone) on potassium uptake in wheat root segments. Phytochemistry 46(2): 245-248.

313. Finotti, E., Di Majo, D. (2003). Influence of solvents on the antioxidant property of flavonoids. Die Nahrung 47(3):186-187.

314. Furuya, H., et al. (2005). Some flavonoids and DHEA-S prevent the cis-effect of expanded CTG repeats in a stable PC12 cell transformant. Biochemical Pharmacology 69(3):503-516.

315. Liu, L., Cheng, Y., Zhang, H. (2004). Phytochemical analysis of anti-atherogenic constituents of Xue-Fu-Zhu-Yu-Tang using HPLC-DAD-ESI-MS. Chemical and Pharmaceutical Bulletin 52(11):1295-1301.

316. Kunizane, H., Ueda, H., Yamazaki, M. (1995). Screening of phagocyte activators in plants; enhancement of TNF production by flavonoids. Yakugaku Zasshi. Journal of the Pharmaceutical Society of Japan 115(9):749-755.

317. Teixeira, S., et al. (2005). Structure-property studies on the antioxidant activity of flavonoids present in diet. Free Radical Biology and Medicine 39(8):1099-1108.

318. Slimestad, R., Fossen, T., Vagen, I.M. (2007). Onions: A source of unique dietary flavonoids. Journal of Agricultural and Food Chemistry 55(25):10067-10080.

319. Seidel, V., Bailleul, F., Waterman, P.G. (2000). Novel oligorhamnosides from the stem bark of Cleistopholis glauca. Journal of Natural Products 63(1):6-11.

320. Hosoi, S., et al. (2006). Structural studies of zoospore attractants from Trachelospermum jasminoides var. pubescens: Taxifolin 3-O-glycosides. Phytochemical Analysis 17(1):20-24.

321. Kim, N.C., et al. (2003). Complete isolation and characterization of sylbins and isosylbins from milk thistle (Sylbum marianum). Organic and Biomolecular Chemistry 1:1684-1689.

322. Minakhmetov, R.A., et al. (2001). Analysis of flavonoids in Silybum marianum fruit by HPLC. Chemistry of Natural Compounds 37(4):318-321.

323. Kiehlmann, E., Slade, P.W. (2003). Methylation of dihydroquercetin acetates: Synthesis of 5-O-methyldihydroquercetin. Journal of Natural Products 66(12): 1562-1566.

324. Bais, H.P., et al. (2003). Structure-dependent phytotoxicity of catechins and other flavonoids: Flavonoid conversions by cell-free protein extracts of Centaurea maculosa (spotted knapweed) roots. Journal of Agricultural and Food Chemistry 51(4):897-901.

325. Delporte, C., et al. (2005). Analgesic-antiinflammatory properties of Proustia pyrifolia. Journal of Ethnopharmacology 99(1):119-124.

326. Lee, E.H., et al. (2003). Constituents of the stems and fruits of Opuntia ficus-indica var. saboten. Archives of Pharmacal Research 26(12):1018-1023.

327. Mbafor, J.T., et al. (1989). Isolation and characterization of taxifolin 6-C-glucoside from Garcinia epunctata. Journal of Natural Products 52(2):417-419.

328. Exarchou, V., et al. (2006). Hyphenated chromatographic techniques for the rapid screening and identification of antioxidants in methanolic extracts of pharmaceutically used plants. Journal of Chromatography. A 1112(1-2):293-302.

329. Sakushima, A., et al. (2002). Separation and identification of taxifolin 3-O-glucoside isomers from Chamaecyparis obtusa (Cupressaceae). Natural Product Letters 16(6):383-387. 
330. Nonaka, G.I., et al. (1987). Tannins and related compounds. LII. Studies on the constituents of the leaves of Thujopsis dolobrata Sieb. et Zucc. Chemical and Pharmaceutical Bulletin 35(3):1105-1108.

331. Dai, S.J., Yu, D.Q. (2005). Studies on the flavonoids in stem of Rhododendron anthopogonoide II. Zhongguo Zhong Yao Za Zhi 30(23):1830-1833.

332. Voirin, B., et al. (1986). Flavonoids from the flowers of Acacia latifolia. Journal of Natural Products 49:943.

333. Pistelli, L., et al. (2000). A new isoflavone from Genista corsica. Journal of Natural Products 63(4):504-506.

334. Prati, S., et al. (2007). Composition and content of seed flavonoids in forage and grain legume crops. Journal of Separation Science 30(4):491-501.

335. Haraguchi, H., et al. (1996). Protection against oxidative damage by dihydroflavonols in Engelhardtia chrysolepis. Bioscience, Biotechnology, and Biochemistry 60(6):945-948.

336. Cai, Y., Chen, T., Xu, Q. (2003). Astilbin suppresses collagen-induced arthritis via the dysfunction of lymphocytes. Inflammation Research 52(8):334-340.

337. Chen, L., et al. (2007). Simultaneous quantification of five major bioactive flavonoids in Rhizoma smilacis glabrae by high-performance liquid chromatography. Journal of Pharmaceutical and Biomedical Analysis 43(5):1715-1720.

338. Chen, T., et al. (1999). A new flavanone isolated from Rhizoma smilacis glabrae and the structural requirements of its derivatives for preventing immunological hepatocyte damage. Planta Medica 65(1):56-59.

339. Du, Q., Li, L., Jerz, G. (2005). Purification of astilbin and isoastilbin in the extract of smilax glabra rhizome by high-speed counter-current chromatography. Journal of Chromatography. A 1077(1):98-101.

340. Li, Y.Q., et al. (1996). Studies on the structure of isoastilbin. Yao Xие Хue Bao 31(10):761-763.

341. Fukunaga, T., et al. (1989). Studies on the constituents of Japanese mistletoes from different host trees, and their antimicrobial and hypotensive properties. Chemical and Pharmaceutical Bulletin 37(6):1543-1546.

342. Messanga, B., Sondengam, B., Bodo, B. (2000). Calodendroside A: A taxifolin diglucoside from the stem bark of Ochna calodendron. Canadian Journal of Chemistry 78:487-489.

343. Japón-Luján, R., Luque De Castro, M.D. (2007). Static-dynamic superheated liquid extraction of hydroxytyrosol and other biophenols from alperujo (a semisolid residue of the olive oil industry). Journal of Agricultural and Food Chemistry 55(9):3629-3634.

344. Brignolas, F., et al. (1995). Induced responses in phenolic metabolism in two Norway spruce clones after wounding and inoculations with Ophiostoma polonicum, a bark beetle-associated fungus. Plant Physiology 109(3):821-827.

345. Lundgren, L.N., Theander, O. (1988). Cis- and trans-dihydroquercetin glucosides from needles of Pinus sylvestris. Phytochemistry 27(3):829-832.

346. Saleem, A., Kivela, H., Pihlaja, K. (2003). Antioxidant activity of pine bark constituents. Zeitschrift fur Naturforschung. Section C. Biosciences 58(5-6): 351-354.

347. Trebatická, J., et al. (2006). Treatment of ADHD with French maritime pine bark extract, Pycnogenol ${ }^{\circledR}$. European Child and Adolescent Psychiatry 15:329-335. 
348. Ohmura, W., et al. (2002). Hydrothermolysis of flavonoids in relation to steaming of Japanese larch wood. Holzforschung 56(5):493-497.

349. Willfor, S.M., et al. (2003). Antioxidant activity of knotwood extractives and phenolic compounds of selected tree species. Journal of Agricultural and Food Chemistry 51(26):7600-7606.

350. Tsydendambaev, P.B., et al. (2007). High-performance liquid chromatographic method for the determination of dihydroquercetin in extracts of medicinal plants. Biomeditsinskaia Khimiia 53(2):212-215.

351. Miyazawa, M., Tamura, N. (2007). Inhibitory compound of tyrosinase activity from the sprout of Polygonum hydropiper L. (Benitade). Biological and Pharmaceutical Bulletin 30(3):595-597.

352. Morimura, K., et al. (2006). 5-O-glucosyldihydroflavones from the leaves of Helicia cochinchinensis. Phytochemistry 67(24):2681-2685.

353. Shao, B., et al. (2007). Simultaneous determination of six major stilbenes and flavonoids in Smilax china by high performance liquid chromatography. Journal of Pharmaceutical and Biomedical Analysis 44:737-742.

354. Yi, Y., et al. (1998). Studies on the chemical constituents of Smilax glabra. Acta Pharmacologica Sinica 33(11):873-875.

355. Meyer, P., et al. (1987). A new petunia flower colour generated by transformation of a mutant with a maize gene. Nature 330(6149):677-678.

356. Wang, Y., et al. (2002). Studies on the chemical constituents from Ampelopsis grossedentata. Zhong Yao Cai 25(4):254-256.

357. Souquet, J.M., et al. (2000). Phenolic composition of grape stems. Journal of Agricultural and Food Chemistry 48(4):1076-1080.

358. Baderschneider, B., Winterhalter, P. (2001). Isolation and characterization of novel benzoates, cinnamates, flavonoids, and lignans from Riesling wine and screening for antioxidant activity. Journal of Agricultural and Food Chemistry 49(6): 2788-2798.

359. Pozo-Bayon, M.A., et al. (2003). Study of low molecular weight phenolic compounds during the aging of sparkling wines manufactured with red and white grape varieties. Journal of Agricultural and Food Chemistry 51(7):2089-2095.

360. Tiukavkina, N.A., Rulenko, I.A., Kolesnik Iu, A. (1997). Dihydroquercetin-A new antioxidant and biologically active food additive. Voprosy Pitaniia 6:12-15.

361. Towatari, K., et al. (2002). Polyphenols from the heartwood of Cercidiphyllum japonicum and their effects on proliferation of mouse hair epithelial cells. Planta Medica 68:995-998.

362. Moriguchi, T., et al. (2002). Flavonol synthase gene expression during citrus fruit development. Physiologia Plantarum 114(2):251-258.

363. Matsuda, M., et al. (2008). Biotransformation of (+)-catechin into taxifolin by a two-step oxidation: Primary stage of $(+)$-catechin metabolism by a novel (+)-catechin-degrading bacteria, Burkholderia sp. KTC-1, isolated from tropical peat. Biochemical and Biophysical Research Communications 366(2):414-419.

364. Kiehlmann, E., Edmond, P.M.L.(1995). Isomerization of dihydroquercetin.Journal of Natural Products 58(3):450-455.

365. Düweler, K.G., Rohdewald, P. (2000). Urinary metabolites of French maritime pine bark extract in humans. Die Pharmazie 55(5):364-368. 
366. Grimm, T., et al. (2006). Single and multiple dose pharmacokinetics of maritime pine bark extract (Pycnogenol) after oral administration to healthy volunteers. BMC Clinical Pharmacology 6:4.

367. Schneider, H., Blaut, M. (2000). Anaerobic degradation of flavonoids by Eubacterium ramulus. Archives of Microbiology 173(1):71-75.

368. Schoefer, L., et al. (2003). Anaerobic degradation of flavonoids by Clostridium orbiscindens. Applied and Environmental Microbiology 69(10):5849-5854.

369. Nielsen, S.E., et al. (1998). In vitro biotransformation of flavonoids by rat liver microsomes. Xenobiotica 28(4):389-401.

370. Svobodova, A., Walterova, D., Psotova, J. (2006). Influence of silymarin and its flavonolignans on $\mathrm{H}(2) \mathrm{O}(2)$-induced oxidative stress in human keratinocytes and mouse fibroblasts. Burns 32(8):973-979.

371. Vitrac, X., et al. (2002). Direct liquid chromatographic analysis of resveratrol derivates and flavanonols in wines with absorbance and fluorescence detection. Analytica Chimica Acta 458:103-110.

372. Trouillas, P., et al. (2004). A theorical study of the conformational behavior and electronic structure of taxifolin correlated with the free radical-scavenging activity. Food Chemistry 88(4):571-582.

373. Theriault, A., et al. (2000). Modulation of hepatic lipoprotein synthesis and secretion by taxifolin, a plant flavonoid. Journal of Lipid Research 41(12): 1969-1979.

374. Braca, A., et al. (2002). Antioxidant activity of flavonoids from Licania licaniaeflora. Journal of Ethnopharmacology 79(3):379-381.

375. Miyazawa, M., Kinoshita,H., Okuno, Y.(2003). Antimutagenic activity of sakuranetin from Prunus Jamasakura. Journal of Food Science: Food Chemistry and Toxicology 68(1):52-56.

376. Rivero-Cruz, I., et al. (2005). Antimycobacterial agents from selected Mexican medicinal plants. Journal of Pharmacy and Pharmacology 57(9):1117-1126.

377. Rakwal, R., et al. (2000). Naringenin 7-O-methyltransferase involved in the biosynthesis of the flavanone phytoalexin sakuranetin from rice (Oryza sativa L.). Plant Science 155(2):213-221.

378. Tuchinda, P., et al. (2002). Antiinflammatory cyclohexenyl chalcone derivatives in Boesenbergia pandurata. Phytochemistry 59(2):169-173.

379. Orjala, J., et al. (1994). Cytotoxic and antibacterial dihydrochalcones from Piper aduncum. Journal of Natural Products 57(1):18-26.

380. Danelutte, A.P., et al. (2003). Antifungal flavanones and prenylated hydroquinones from Piper crassinervium Kunth. Phytochemistry 64(2):555-559.

381. Jung, Y.H., et al. (2005). The rice (Oryza sativa) blast lesion mimic mutant, blm, may confer resistance to blast pathogens by triggering multiple defense-associated signaling pathways. Plant Physiology and Biochemistry 43(4):397-406.

382. Aida, Y., et al. (1996). Synthesis of 7-methoxyapigeninidin and its fungicidal activity against Gloeocercospora sorghi. Bioscience, Biotechnology, and Biochemistry 60(9):1495-1496.

383. Kodama, O., et al. (1992). Sakuranetin, a flavonone phytoalexin from ultravioletirradiated rice leaves. Phytochemistry 31(11):3807-3809. 
384. Rakwal, R., Hasegawa, M., Kodama, O. (1996). A methyltransferase for synthesis of the flavanone phytoalexin sakuranetin in rice leaves. Biochemical and Biophysical Research Communications 222(3):732-735.

385. Tamogami, S., Rakwal, R., Kodama, O. (1997). Phytoalexin production by amino acid conjugates of jasmonic acid through induction of naringenin-7-Omethyltransferase, a key enzyme on phytoalexin biosynthesis in rice (Oryza sativa L.). FEBS Letters 401(2-3):239-242.

386. Jung, Y.H., et al. (2006). Differential expression of defense/stress-related marker proteins in leaves of a unique rice blast lesion mimic mutant (blm). Journal of Proteome Research 5(10):2586-2598.

387. Rojas, A., et al. (1996). Smooth muscle relaxing compounds from Dodonaea viscosa. Planta Medica 62(2):154-159.

388. Abdel-Sattar, E., et al. (2000). Phenolic compounds from Eucalyptus maculata. Die Pharmazie 55(8):623-624.

389. Lambert, J.D., et al. (2005). Cytotoxic lignans from Larrea tridentata. Phytochemistry 66(7):811-815.

390. Moreira, D.D., Guimaraes, E.F., Kaplan, M.A. (2000). A C-glucosylflavone from leaves of Piper lhotzkyanum. Phytochemistry 55(7):783-786.

391. Jerz, G., Waibel, R., Achenbach, H. (2005). Cyclohexanoid protoflavanones from the stem-bark and roots of Ongokea gore. Phytochemistry 66(14):1698-1706.

392. Ghisalberti, E.L., et al. (1978). Constituents of propolis. Cellular and Molecular Life Sciences 34(2):157-158.

393. Tohno, H., et al. (2010). Evaluation of estrogen receptor beta binding of pruni cortex and its constituents. Yakugaku Zasshi. Journal of the Pharmaceutical Society of Japan 130(7):989-997.

394. Duewell, H. (1978). Chemotaxonomy of hte genus Xanthorrhoea. Biochemical Systematics and Ecology 25(8):717-738.

395. Asahina, Y. (1908). Sakuranetin, a new glycoside from the bark of Prunus pseudocerasus. Archiv der Pharmazie 246:259-272.

396. Obara, N., Hasegawa, M., Kodama, O. (2002). Induced volatiles in elicitor-treated and rice blast fungus-inoculated rice leaves. Bioscience, Biotechnology, and Biochemistry 66(12):2549-2559.

397. Kim, B.G., et al. (2006). Regiospecific flavonoid 7-O-methylation with Streptomyces avermitilis O-methyltransferase expressed in Escherichia coli. Journal of Agricultural and Food Chemistry 54(3):823-828.

398. Mori, M., et al. (2007). Isolation and molecular characterization of a spotted leaf 18 mutant by modified activation-tagging in rice. Plant Molecular Biology 63(6):847-860.

399. Nakazato, Y., et al. (2000). Methionine-induced phytoalexin production in rice leaves. Bioscience, Biotechnology, and Biochemistry 64(3):577-583.

400. Tamogami, S., Kodama, O. (2000). Coronatine elicits phytoalexin production in rice leaves (Oryza sativa L.) in the same manner as jasmonic acid. Phytochemistry 54(7):689-694.

401. BCC Research, Market Forecasting. (2003). Nutraceuticals sales to hit $\$ 75$ billion. Accessed February 24, 2009. http://www.nutraingredients-usa.com/ConsumerTrends/Nutraceuticals-sales-to-hit-75-billion. 
402. Werner, R.A., et al. (2004). Biosynthesis of gallic acid in Rhus typhina: Discrimination between alternative pathways from natural oxygen isotope abundance. Phytochemistry 65(20):2809-2813.

403. Niemetz, R., Gross, G.G. (2005). Enzymology of gallotannin and ellagitannin biosynthesis. Phytochemistry 66(17):2001-2011.

404. Faried, A., et al. (2007). Anticancer effects of gallic acid isolated from Indonesian herbal medicine, Phaleria macrocarpa (Scheff.) Boerl, on human cancer cell lines. International Journal of Oncology 30(3):605-613.

405. Gross, G.G. (1983). Partial purification and properties of UDP-glucose: Vanillate 1-O-glucosyl transferase from oak leaves. Phytochemistry 22(10):2179-2182.

406. Ling, S.K., Tanaka, T., Kouno, I. (2002). New cyanogenic and alkyl glycoside constituents from Phyllagathis rotundifolia. Journal of Natural Products 65(2): 131-135.

407. Miyaichi, Y., et al. (2006). Studies on nepalese crude drugs. XXVIII. Chemical constituents of Bhote Khair, the underground parts of Eskemukerjea megacarpum Hara. Chemical and Pharmaceutical Bulletin 54(1):136-138.

408. Niemetz, R., Gross, G.G. (2001). Gallotannin biosynthesis: Beta-glucogallin: Hexagalloyl 3-O-galloyltransferase from Rhus typhina leaves. Phytochemistry 58(5): 657-661.

409. Subeki, S., et al. (2005). Anti-babesial and anti-plasmodial compounds from Phyllanthus niruri. Journal of Natural Products 68(4):537-539. 
